OPEN ACCESS

Edited by:

Ilaria Puxeddu,

University of Pisa, Italy

Reviewed by:

Antonio Molino,

University of Naples Federico II, Italy

Koichiro Asano,

Tokai University, Japan

*Correspondence:

Paige Lacy

placy@ualberta.ca

Manali Mukherjee

mukherj@mcmaster.ca

Specialty section: This article was submitted to Inflammation Pharmacology,

a section of the journal

Frontiers in Pharmacology

Received: 12 October 2021 Accepted: 10 December 2021

Published: 19 January 2022

Citation:

Salter $B$, Lacy $P$ and Mukherjee $M$ (2022) Biologics in Asthma: A

Molecular Perspective to

Precision Medicine.

Front. Pharmacol. 12:793409.

doi: 10.3389/fphar.2021.793409

\section{Biologics in Asthma: A Molecular Perspective to Precision Medicine}

\author{
Brittany Salter ${ }^{1,2}$, Paige Lacy ${ }^{3 *}$ and Manali Mukherjee ${ }^{1,2 *}$ \\ ${ }^{1}$ Division of Respirology, Department of Medicine, McMaster University, Hamilton, ON, Canada, ${ }^{2}$ Firestone Institute for \\ Respiratory Health, St. Joseph's Healthcare, Hamilton, ON, Canada, ${ }^{3}$ Department of Medicine, University of Alberta, Edmonton, \\ $A B$, Canada
}

Recent developments in therapeutic strategies have provided alternatives to corticosteroids as the cornerstone treatment for managing airway inflammation in asthma. The past two decades have witnessed a tremendous boost in the development of anti-cytokine monoclonal antibody $(\mathrm{mAb})$ therapies for the management of severe asthma. Novel biologics that target eosinophilic inflammation (or type 2, T2 inflammation) have been the most successful at treating asthma symptoms, though there are a few in the drug development pipeline for treating non-eosinophilic or T2low asthma. There has been significant improvement in clinical outcomes for asthmatics treated with currently available monoclonal antibodies (mAbs), including antiimmunoglobulin (Ig) E, anti-interleukin (IL)-4 receptor a subunit, anti-IL-5, anti-IL-5Ra, anti-IL-6, anti-IL-33, and anti-thymic stromal lymphopoietin (TSLP). Despite these initiatives in precision medicine for asthma therapy, a significant disease burden remains, as evident from modest reduction of exacerbation rates, i.e., approximately 40-60\%. There are numerous studies that highlight predictors of good responses to these biologics, but few have focused on those who fail to respond adequately despite targeted treatment. Phenotyping asthmatics based on blood eosinophils is proving to be inadequate for choosing the right drug for the right patient. It is therefore pertinent to understand the underlying immunology, and perhaps, carry out immune endotyping of patients before prescribing appropriate drugs. This review summarizes the immunology of asthma, the cytokines or receptors currently targeted, the possible mechanisms of suboptimal responses, and the importance of determining the immune make-up of individual patients prior to prescribing mAb therapy, in the age of precision medicine for asthma.

Keywords: severe asthma, monoclonal antibodies, T2 inflammation, eosinophil, treatment response

\section{INTRODUCTION}

Asthma is defined by reversible airflow obstruction, hyperresponsiveness, and inflammation, that manifests as wheeze, dyspnea, and cough. Despite a wide array of treatments available for asthma, $5-10 \%$ of patients have poor response to inhaled corticosteroids, and remain on high doses of systemic corticosteroids (Heffler et al., 2019). At first glance, this may seem like a non-significant percentile; however this subgroup contributes substantially to the economical disease burden, accounting for 56 billion US dollars annually, due to frequent exacerbations with need for acute care (Barnett and Nurmagambetov, 2011). Current clinical guidelines for asthma diagnosis include assessment of lung function through spirometry with or without a bronchoprovocation challenge to 
quantify hyperresponsiveness. Interestingly, despite airway inflammation being a hallmark feature of asthma, it is not a requirement for asthma diagnosis, but instead helps to stratify disease severity. As a whole, the current tests we have do not account for the vast immunological heterogeneity of asthma.

We have seen great strides over the past 2 decades with respect to the development of alternative therapies to corticosteroids. The era of monoclonal antibodies (mAbs) targeting receptors and cytokines involved in the pathogenesis of asthma has emerged in severe asthma management. Although we have witnessed significant improvement in clinical outcomes for severe asthmatics treated with currently available mAbs, there still remains a proportion of patients with refractory disease. Biologic therapy has used biomarkers to phenotype patients and identify those who would benefit most from therapy, using blood eosinophils, serum total IgE and periostin, and fraction of excreted nitric oxide (FeNO). Unfortunately, these biomarkers fail to reflect the complexity of underlying inflammatory endotypes, and are proving to be inadequate for not only choosing the right drug for the right patient, but also monitoring response to treatment. It has been clear that inflammation in severe asthma is not always characterized by the presence of eosinophilia. We need to pay closer attention to the patients who fail to respond to mAbs to learn lessons on how to better individualize treatment. Immunological endotyping has been proposed as a potential tool to curtail treatment for individual patient's and needs to be further studied. This review will summarize the immunology of asthma, the cytokines or receptors currently targeted, and potential mechanisms of sub-optimal responses.

\section{AIRWAY INFLAMMATION IN ASTHMA}

Asthma was initially categorized into two simple phenotypes of allergic and non-allergic disease, however over time our understanding of disease pathogenesis has expanded, and we now categorize phenotypes based on underlying inflammatorybased mechanisms (neutrophilic, eosinophilic, mixed, and paucigranulocytic). There is evidence to suggest that even with each inflammatory phenotype there is a great deal of heterogeneity, with several different immune endotypes contributing to the overlying inflammation. Broadly, there are two asthma endotypes characterized as type 2 (T2) high and T2 low inflammation. The T2-high endotype, defined by a T2 cytokine response (IL-4, IL-5, and IL-13), is the most common endotype and the most well understood.

In order to individualize treatment, a patient's asthma endotype must be identified and fortunately, genomics has emerged as a powerful tool for diagnosis. In severe asthma, three transcriptome-associated clusters (TACs) have been identified, including TAC 1 (IL-33R, CCR3, TSLPR), TAC2 (interferon-, tumour necrosis factor alpha-, and inflammasomeassociated genes), and TAC3 (genes of metabolic pathways, ubiquitination and mitochondrial function). TAC1 has the highest enrichment of gene signatures for IL-13/Th2-high and innate lymphoid cell type 2 (ILC2) inflammation, along with the highest sputum and blood eosinophils and serum periostin.
Furthermore, this cluster has oral corticosteroid (OCS)dependency, frequent exacerbations, and severe airflow obstruction. Conversely, TAC2 has high sputum neutrophils and TAC3 has normal to high sputum eosinophilia and better preserved $\mathrm{FEV}_{1}$, with the least OCS-dependency. As such, in the setting of severe asthma, three unique clusters of gene expression have been identified, further demonstrating the heterogeneity of endotypes within each inflammatory phenotype.

\subsection{Type 2 Inflammation}

T2-high inflammation develops in response to cross-talk between innate and adaptive immune responses. Allergic asthma is triggered by inhaled allergens that are taken up within the airways by antigen presenting cells, including dendritic cells. These cells go on to process aeroallergens and present antigen peptides on their cell surface via the HLA class II molecule of the major histocompatibility complex (MHC Class II) within lymph nodes. MHC class II interacts with the $\mathrm{T}$ cell receptor (TCR) of naive $\mathrm{CD} 4^{+} \mathrm{T}$ cells, resulting in polarization towards the T helper 2 (Th2) lineage. Polarization is, in part, driven by IL-4, produced by neighbouring mast cells and basophils.

Once Th2 cells have matured, they migrate to the airways where further antigen exposure results in TCR-antigen binding and prompts Th2 cells to release T2 cytokines including IL-4, IL5 , and IL-13, leading to downstream airway inflammation (Hammad and Lambrecht, 2021). IL-4 and IL-13 induce Ig class switching of $\mathrm{B}$ cells to produce $\operatorname{IgE}$, which has the capacity to bind to and activate high-affinity FceR1 receptors on mast cells and basophils. After initial sensitization, reexposure to allergen results in IgE crosslinking with FceR1 receptors, leading to mast cell and basophil degranulation of histamine, leukotrienes, and prostaglandins, which go on to promote bronchoconstriction.

This aforementioned adaptive immune system, is also triggered by upstream innate processes. Inhaled antigens interact with airway epithelium, resulting in production of alarmins including, thymic stromal lymphopoietin (TSLP), IL25, and IL-33 (Hammad and Lambrecht, 2021). Collectively, these alarmins promote the release of cytokines from Th2 cells, basophils, mast cells, and ILC2s (Salter et al., 2019). Similar to Th2 cells, ILC2s are potent promoters of T2-high inflammation, through production of IL-5 and IL-13 (Salter et al., 2019). In addition, basophils and mast cells, have been identified as potent sources of IL-4 and IL-13 (Bao and Reinhardt, 2015). With respect to IL-13, this cytokine also plays a role in inducing mucus production, airway remodeling, and hyperresponsiveness. In particular, for quite some time the spotlight has been on IL-5, for its an important role in asthma. This eosinophil-maturing cytokine is produced not only by Th2 cells, but also granulocytes and ILC2s. The biologic effects of IL-5 are mediated through interaction with IL-5Ra and a non-specific beta chain heterodimer, recognized by IL-3 and GM-CSF (Rossjohn et al., 2000). When IL-5 is present it binds to IL-5Ra and drives formation of a functional IL-5R $\alpha / \beta$ chain receptor complex, that promotes activation of an intricate network of signaling pathways (Johanson et al., 1995). 
IL-5Ra is highly expressed on eosinophils (Varricchi et al., 2016) and the interaction between IL-5 and IL-5Ra results in downstream activation of intracellular signaling proteins JAK2 and STAT 1, 3, and 5, which in turn stimulate transcriptional factors involved in eosinophil proliferation (Pazdrak et al., 1995). JAK2 is also involved in the inhibition of eosinophil apoptosis through the active cooperation with Lyn and Raf-1 kinases (Pazdrak et al., 1995; Schwartz et al., 2015). Other signal transduction molecules that are activated by IL- 5 include phosphoinositide 3-kinase (PI3K) and mitogen-activated protein kinases (MAPK). Through activation of extracellular signal-regulated kinases (ERK) $1 / 2$ and protein kinase $C(\mathrm{PKC})$, PI3K mediates IL-5-induced interaction of eosinophils with intracellular adhesion molecule-1 (ICAM-1) (Sano et al., 2005). The Ras-Raf-1-mediated activation of the ERK subfamily of MAPK drives c-fos gene transcription, which is involved in promoting cell maturation, survival, and proliferation (Adachi et al., 2000). Lastly, through a NF-kB-dependent mechanism, p38 MAPK up-regulates eosinophil proinflammatory cytokine production (Adachi et al., 2000). IL-5 is responsible for the activation of many integral functions of eosinophils, including maturation, accumulation and activation, and action depends on the interaction with IL-5Ra, mAbs have been developed against IL-5 and IL-5Ra.

Eosinophils exert their effects on the airways through degranulation (principally piecemeal degranulation) including the release of free intact eosinophilic granules (FEGs), which produce tissue-damaging eosinophil granule proteins, including major basic protein $(\mathrm{MBP})$, eosinophilia cationic protein (ECP), eosinophilic-derived neurotoxin (EDN), and eosinophil peroxidase (EPX) (Hogan et al., 2008). Eosinophils also release extracellular DNA deposits that form a web-like structure called eosinophilic extracellular traps (EETs) through a process called ETosis (Mukherjee et al., 2018a). EETs have an autocrine effect on promoting eosinophil degranulation and inducing epithelial cells to produce IL-6 and IL-8 (Mukherjee et al., 2018a). Collectively, the aforementioned mediators contribute to airway remodeling, airway hyperreactivity, and increased mucus production.

\subsection{Type 1 Inflammation}

T2-low inflammation has emerged as another pathway that results in asthma pathogenesis. Pattern recognition receptors (PRRs) on surface of airway epithelial cells, granulocytes, dendritic cells, and $\mathrm{T}$ cells, act to recognize danger-associated molecular patterns (DAMPs) and pathogen-associated molecular patterns (PAMPs) and induce downstream mediator release. Activation of the PRR called nucleotide-binding oligomerization domain-like receptor (NLR) results in stimulation of inflammasomes, which are multimolecular signaling platforms that act as critical inducers of host defense (Elliott and Sutterwala, 2015). In particular, the NLRP3 inflammasome is one of the five major inflammosomes that induces secretion of IL-1 $\beta$ (Elliott and Sutterwala, 2015). This secretion is mediated through caspase- 1 , which cleaves IL- $1 \beta$ into its secretory isoform. IL- $1 \beta$ plays a role in Th17 differentiation and IL-17 production. IL-17 acts as an important mediator of neutrophilic inflammation and is elevated in severe asthmatics with frequent exacerbations (Ricciardolo et al., 2017). The inflammasome promotes pyroptosis, a form of lytic cell death. NLRP3, caspase-1, and IL-1 $\beta$ are increased in sputa of severe asthmatics and correlate with disease severity (Simpson et al., 2014; Kim et al., 2017). Neutrophils have been proposed to play a role in activation of the inflammasome (Wright et al., 2016). Neutrophil-derived extracellular DNA (eDNA) is released in a web-like structure to form neutrophil extracellular traps (NETs), in a process known as NETosis, which can be induced by infectious and non-infectious conditions. The presence of airway neutrophilia and NETosis results in inflammasome activation, leading to promotion of Th17-mediated inflammation. Although there are emerging biologic therapies that target T2-low inflammation, the overall identification of this endotype and use of biomarkers to monitor treatment response remains largely unknown.

\section{CURRENTLY AVAILABLE BIOLOGICAL THERAPY AND SUBOPTIMAL RESPONSES IN SEVERE ASTHMA}

The practice of precision medicine in asthma is far from optimal due to lack of complete understanding of the complex immunological nature of asthma. The severe asthma group is quite heterogeneous in nature and as such, a "one size fits all" approach cannot be used to manage these patients. Although many severe asthmatics have T2-high inflammation, the underlying mechanisms driving this inflammation may vary drastically across patients. This problem has been underscored by the high degree of variation in patient response to biologic therapy, where some patients respond dramatically and others either fail treatment or have suboptimal responses. Superresponders (SR) are defined as having improvement across three or more domains over a 12-month period including exacerbation elimination and improvement in asthma control (Upham et al., 2021). A better understanding is needed on how to identify these SR and determine what characteristics predispose them to a dramatic response to biologic therapy. Similarly, we need to better identify treatment failures and suboptimal responders to determine what underlying mechanisms contribute to this and how they differ from SR. In this section, we will review evidence behind current biologics and potential underlying mechanisms accountable for suboptimal response and treatment failure. The targeted pathways along with the key studies pertaining to these biologics are summarized in Figure 1 and Tables 1-7 respectively, while Figure 2 summarises the possible factors associated/contributing to poor therapeutic responses.

\subsection{IgE Targeted Therapy}

$\operatorname{IgE}$ is the primary immunoglobulin involved in T2-high inflammation. Omalizumab binds to the third constant region of IgE and prevents free IgE from interacting with high and lowaffinity FceR1 receptors (Fahy et al., 1997). As a result of this binding, free serum IgE levels decrease, as well as the overall IgE receptor density on mast cells and basophils. Numerous 


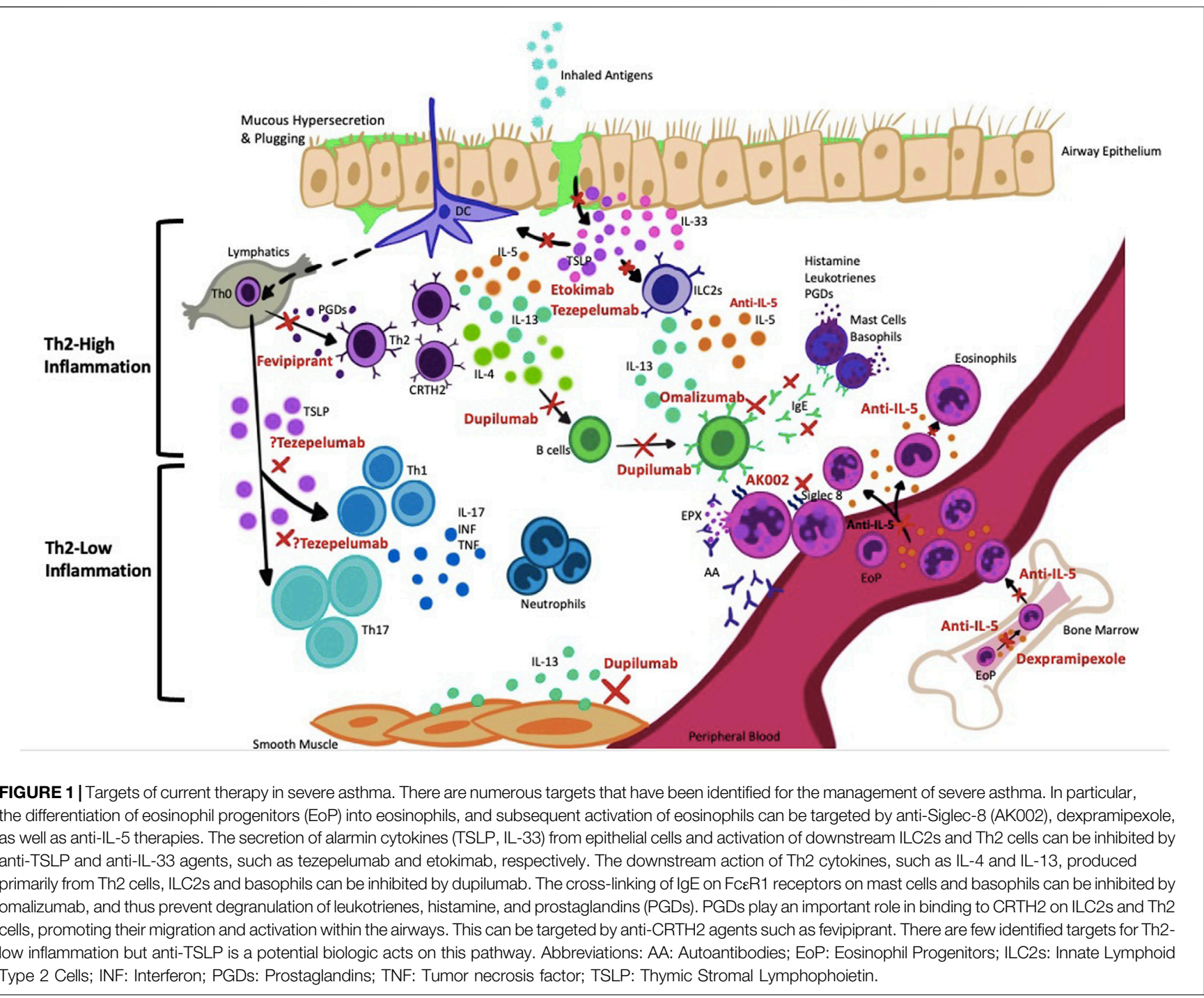

randomized clinical trials (RCTs) and real-life studies have shown that treatment of asthmatics with omalizumab results in a dosedependent reduction in free IgE in serum, improvement in lung function, and modest reduction in exacerbation rates, as well as emergency visits and hospitalizations (Corne et al., 1997; Hanania et al., 2011; Rodrigo et al., 2011; Normansell et al., 2014). RCTs have also shown improvement in symptom control, quality of life, and reduced oral corticosteroid (OCS) use (Table 1) (Humbert et al., 2005; Rodrigo et al., 2011; Normansell et al., 2014; Pelaia et al., 2018a). In terms of molecular findings, omalizumab reduces both eosinophil and basophil infiltration within the airways (Rodrigo et al., 2011; Normansell et al., 2014; Pelaia et al., 2018a). Of note, a large retrospective analysis of 25 RCTs demonstrated greater reduction in asthma exacerbation patients who specifically had high blood eosinophilia and fractional exhaled nitric oxide (FeNO) levels, which was suggestive of eosinophilic inflammation (Hanania et al., 2013). Based on these findings, it seemed justified to prescribe anti-IgE biologics to severe asthmatics with evidence of atopy. As promising as the aforementioned studies are, they were primarily based on mild-moderate asthmatics, and more studies are needed to determine efficacy of these agents in severe corticosteroid-dependent asthma.

\subsubsection{Possible Reasons for Suboptimal Responses With Anti-lgE}

Agents that target IgE-dependent mechanisms have been shown to be efficacious in mild to moderate asthma, however, this pathway may not be the major driver of eosinophilic inflammation in severe asthma. Thus, although anti-IgE agents may sufficiently suppress IgE-dependent mechanisms, the IgEindependent pathways are still active within the airways, continuing to drive Th2 inflammation. This is, in part, supported by studies showing that omalizumab treatment in severe asthma does not reduce sputum eosinophils, (Mukherjee et al., 2019) and that pediatric patients with severe asthma are low responders to omalizumab (Garcia et al., 2013). Chapman et al. (2019) assessed the efficacy of using mepolizumab 
TABLE 1 | Summary of anti-lgE targeted randomized clinical trials in severe asthma.

\begin{tabular}{|c|c|c|c|c|c|c|}
\hline \multicolumn{7}{|l|}{ Anti-IgE } \\
\hline $\begin{array}{l}\text { Landmark study } \\
\text { and year }\end{array}$ & Study type & $\begin{array}{l}\text { Asthma } \\
\text { severity }\end{array}$ & $\begin{array}{c}\text { Asthma } \\
\text { phenotype }\end{array}$ & $\begin{array}{l}\text { Dosing, duration } \\
\text { and route } \\
\text { of administration }\end{array}$ & Clinical effect & Molecular effect \\
\hline Hanania et al. (2013) & Phase 2 & $\begin{array}{l}\text { Severe } \\
\text { uncontrolled } \\
\text { asthma }\end{array}$ & Atopic & $\begin{array}{l}\text { Dose: } 0.016 \mathrm{mg} / \mathrm{kg} \mathrm{lgE} \\
\text { (IU/ml) } \\
\text { Frequency: Q2W, Q4W } \\
\text { Route: SC } \\
\text { Duration: } 48 \mathrm{~W}\end{array}$ & $\begin{array}{l}\text {-Reduced AAER } \\
\text {-Improved AQLQ, FEV } 1\end{array}$ & -Reduced FeNO \\
\hline $\begin{array}{l}\text { INNOVATE, } \\
2004 \text { Busse et al. } \\
(2019)\end{array}$ & Phase 2 & $\begin{array}{l}\text { Severe } \\
\text { uncontrolled } \\
\text { asthma }\end{array}$ & Atopic & $\begin{array}{l}\text { Dose: } \\
\text { 0.008-0.016 mg/kg lgE } \\
\text { (IU/ml) } \\
\text { Frequency: Q2W, Q4W } \\
\text { Route: SC } \\
\text { Duration: } 28 \mathrm{~W}\end{array}$ & $\begin{array}{l}\text {-Reduced AAER, ED visits } \\
\text {-Improved morning PEF }\end{array}$ & $-N / A$ \\
\hline Busse et al. (2001) & Phase 3 & $\begin{array}{l}\text { Severe } \\
\text { uncontrolled } \\
\text { asthma }\end{array}$ & Atopic & $\begin{array}{l}\text { Dose: } \\
\text { 0.008-0.016 mg/kg lgE } \\
\text { (IU/ml) } \\
\text { Frequency: Q4W } \\
\text { Route: SC } \\
\text { Duration: } 28 \mathrm{~W}\end{array}$ & $\begin{array}{l}\text {-Reduced AAER, steroid } \\
\text { dose } \\
\text {-Improved morning } \\
\text { PEF, ACQ }\end{array}$ & -Reduced serum lgE \\
\hline Garcia et al. (2013) & Phase 3b & $\begin{array}{l}\text { Severe } \\
\text { uncontrolled } \\
\text { asthma }\end{array}$ & Atopic & $\begin{array}{l}\text { Dose: 200, } 300 \text { mg } \\
\text { Frequency: Q2W } \\
\text { Route: SC } \\
\text { Duration: } 16 \mathrm{~W}\end{array}$ & $\begin{array}{l}\text {-No change in ACQ or AAER } \\
\text {-Increased FEV } 1\end{array}$ & $\begin{array}{l}\text {-FceR1 decreased on } \\
\text { basophils and DCs at } 16 \mathrm{~W}\end{array}$ \\
\hline Djukanović et al. (2004) & Phase 3 & $\begin{array}{l}\text { Severe } \\
\text { uncontrolled } \\
\text { asthma }\end{array}$ & Atopic & $\begin{array}{l}\text { Dose: } 0.016 \mathrm{mg} / \mathrm{kg} \mathrm{lgE} \\
\text { (IU/ml) } \\
\text { Frequency: Q4W } \\
\text { Route: SC } \\
\text { Duration: } 16 \mathrm{~W}\end{array}$ & -No improvement in AHR & $\begin{array}{l}\text {-Reduced SP, submucosal } \\
\text { and epithelial eosinophils } \\
\text {-Reduced FceR1+ and IgE + } \\
\text { cells, CD4/CD3/CD8 T cells, } \\
\text { and IL-4+ cells in submucosa } \\
\text {-Reduced serum IgE }\end{array}$ \\
\hline Chanez et al. (2010) & Phase 3 & $\begin{array}{l}\text { Severe } \\
\text { uncontrolled } \\
\text { asthma }\end{array}$ & Atopic & $\begin{array}{l}\text { Dose: } 0.016 \text { mg/kg lgE } \\
\text { (IU/ml) } \\
\text { Frequency: Q4W } \\
\text { Route: SC } \\
\text { Duration: } 16 \mathrm{~W}\end{array}$ & -No change in AAER & $\begin{array}{l}\text {-Reduced FceR1 on basophils } \\
\text { and DCs at } 16 \mathrm{~W}\end{array}$ \\
\hline de Llano et al. (2013) & Phase 3 & $\begin{array}{l}\text { Severe } \\
\text { uncontrolled } \\
\text { asthma }\end{array}$ & $\begin{array}{l}\text { Atopic and } \\
\text { non-atopic }\end{array}$ & $\begin{array}{l}\text { Dose: } 0.016 \mathrm{mg} / \mathrm{kg} \mathrm{lgE} \\
\text { (IU/ml) } \\
\text { Frequency: Q4W } \\
\text { Route: SC } \\
\text { Duration: } 24 \mathrm{M}\end{array}$ & $\begin{array}{l}\text {-Improved GETE scale and } \\
\text { ACT score } \\
\text {-Increased FEV } 1 \\
\text {-No change in AAER }\end{array}$ & $-N / A$ \\
\hline $\begin{array}{l}\text { XCLUSIVE, } \\
2011 \text { Schumann et al. } \\
\text { (2012) }\end{array}$ & Phase 3 & $\begin{array}{l}\text { Severe } \\
\text { uncontrolled } \\
\text { asthma }\end{array}$ & Atopic & $\begin{array}{l}\text { Dose: } 0.016 \text { mg/kg lgE } \\
\text { (IU/ml) } \\
\text { Frequency: Q4W } \\
\text { Route: SC } \\
\text { Duration: } 6 \mathrm{M}\end{array}$ & $\begin{array}{l}\text {-Increased } \mathrm{FEV}_{1} \text { at } 16 \mathrm{~W} \\
\text {-Improved } \mathrm{ACQ} \text { at } 16 \mathrm{~W} \\
\text {-Reduced } \mathrm{AAER}\end{array}$ & $-N / A$ \\
\hline Holgate et al. (2005) & Phase 3 & $\begin{array}{l}\text { Severe } \\
\text { uncontrolled } \\
\text { asthma }\end{array}$ & Atopic & $\begin{array}{l}\text { Dose: } 0.016 \mathrm{mg} / \mathrm{kg} \mathrm{lgE} \\
\text { (IU/ml) } \\
\text { Frequency: Q4W } \\
\text { Route: SC } \\
\text { Duration: } 16 \mathrm{~W}\end{array}$ & -Reduced fluticasone dose & $-N / A$ \\
\hline Barnes et al. (2013) & $\begin{array}{l}\text { Retrospective } \\
\text { study }\end{array}$ & $\begin{array}{l}\text { Severe } \\
\text { uncontrolled } \\
\text { asthma }\end{array}$ & Atopic & $\begin{array}{l}\text { Dose: } 0.016 \text { mg/kg lgE } \\
(\mathrm{IU} / \mathrm{ml}) \\
\text { Frequency: Q4W } \\
\text { Route: SC } \\
\text { Duration: } 12 \mathrm{M}\end{array}$ & $\begin{array}{l}\text {-Reduced steroid use/dose, } \\
\text { hospitalizations and ED } \\
\text { visits, and AAER } \\
\text {-Increased FEV } 1\end{array}$ & $-N / A$ \\
\hline
\end{tabular}

AAER: annualized asthma exacerbation ratio; $A C Q$, asthma control questionnaire; $A H R$ : airway hyperresponsiveness; $A C T$, asthma control test; $A Q L Q$, asthma quality of life questionnaire; $D C$, dendritic cell; ED, emergency department; FeNO, fraction of expired nitric oxide; GETE, global evaluation of treatment effectiveness; IU, international units; M, months; NA, not applicable; $Q$, every; SC, subcutaneous; W, weeks. 
TABLE 2 | Summary of randomized clinical trials assessing mepolizumab in severe asthma.

\begin{tabular}{|c|c|c|c|c|c|c|}
\hline \multicolumn{7}{|l|}{ Anti-IL-5: mepolizumab } \\
\hline $\begin{array}{l}\text { Landmark study } \\
\text { and year }\end{array}$ & RCT phase & $\begin{array}{l}\text { Asthma } \\
\text { severity }\end{array}$ & Asthma phenotype & $\begin{array}{l}\text { Dosing, duration } \\
\text { and route } \\
\text { of administration }\end{array}$ & Clinical effect & Molecular effect \\
\hline Nair et al. (2009) & Phase 2 & $\begin{array}{l}\text { Severe } \\
\text { uncontrolled } \\
\text { asthma }\end{array}$ & $\begin{array}{l}\geq 300 \text { cells/ } \mu \mathrm{l} \text { PB } \\
\text { eosinophils in previous } \\
\text { year or } \geq 150 \text { cells/ } / \mathrm{l} \text { PB } \\
\text { eosinophils at screening }\end{array}$ & $\begin{array}{l}\text { Dose: } 750 \mathrm{mg} \\
\text { Route: IV } \\
\text { Frequency: Q4W } \\
\text { Duration: } 26 \mathrm{~W}\end{array}$ & $\begin{array}{l}\text {-Reduced AAER and } \\
\text { time-to-exacerbation } \\
\text {-Reduction in } \\
\text { prednisone dose } \\
\text {-Improved } \mathrm{FEV}_{1} \\
\text { and ACQ }\end{array}$ & $\begin{array}{l}\text {-No exacerbations } \\
\text { associated with SP } \\
\text { eosinophilia, instead there } \\
\text { was SP neutrophilia } \\
\text {-Reduced SP and PB } \\
\text { eosinophils }\end{array}$ \\
\hline Haldar et al. (2009) & & $\begin{array}{l}\text { Severe } \\
\text { uncontrolled } \\
\text { asthma }\end{array}$ & $\begin{array}{l}\geq 3 \% \text { sputum eosinophils } \\
\text { in previous } 2 \text { years }\end{array}$ & $\begin{array}{l}\text { Dose: } 750 \text { mg } \\
\text { Route: IV } \\
\text { Frequency: Q4W } \\
\text { Duration: } 52 \text { W }\end{array}$ & $\begin{array}{l}\text {-57\% reduction in AAER } \\
\text { at } 50 \mathrm{~W} \\
\text {-Improved AQLQ score } \\
\text {-No change in } \mathrm{FEV}_{1} \\
\text { post-BD use or AHR }\end{array}$ & $\begin{array}{l}\text {-Reduced PB and SP } \\
\text { eosinophils } \\
\text {-No change in FeNO or } \\
\text { neutrophil count in SP }\end{array}$ \\
\hline $\begin{array}{l}\text { DREAM, } 2012 \text { Pavord } \\
\text { et al. (2012); Ortega } \\
\text { et al. (2016) }\end{array}$ & Phase 2 & $\begin{array}{l}\text { Severe } \\
\text { uncontrolled } \\
\text { asthma }\end{array}$ & $\begin{array}{l}\geq 0.3 \times 10^{9} \text { cells } / \mathrm{LPB} \\
\text { eosinophils or FeNO } \\
\geq 50 \text { ppb or } \mathrm{SP} \\
\text { eosinophils } \geq 3 \%\end{array}$ & $\begin{array}{l}\text { Dose: 75-750 mg } \\
\text { Route: IV } \\
\text { Frequency: Q4W } \\
\text { Duration: } 52 \text { W }\end{array}$ & $\begin{array}{l}-48 \% \text { reduction in } \\
\text { exacerbations at } 52 \mathrm{~W} \\
-60 \% \text { reduction in } \\
\text { exacerbations requiring } \\
\text { hospitalization or } \mathrm{ED} \\
\text { visits } \\
-\mathrm{No} \text { difference in } \mathrm{AQLQ} \text {, } \\
\mathrm{ACQ} \text { sores or } \mathrm{FEV} \\
\text { A }\end{array}$ & $\begin{array}{l}\text {-Reduced PB and SP } \\
\text { eosinophils }\end{array}$ \\
\hline $\begin{array}{l}\text { MENSA, } 2014 \text { Ortega } \\
\text { et al. (2014); Ortega } \\
\text { et al. (2016) }\end{array}$ & Phase 3 & $\begin{array}{l}\text { Severe } \\
\text { uncontrolled } \\
\text { asthma }\end{array}$ & $\begin{array}{l}\geq 300 \text { cells } / \mu \mathrm{l} \text { PB } \\
\text { eosinophils in previous } \\
\text { year or } \geq 150 \text { cells/ } \mu \mathrm{l} \text { PB } \\
\text { eosinophils at screening }\end{array}$ & $\begin{array}{l}\text { Dose: } 75,100 \text { mg } \\
\text { Route: IV, SC } \\
\text { Frequency: Q4W } \\
\text { Duration: } 32 \mathrm{~W}\end{array}$ & $\begin{array}{l}-53 \% \text { reduction in AAER } \\
\text { at } 32 \mathrm{~W} \\
-61 \% \text { reduction in } \mathrm{ED} \\
\text { visits or hospitalizations } \\
\text { at } 32 \mathrm{~W} \\
\text {-Improved } \mathrm{FEV}_{1}, \mathrm{PEF}, \\
\mathrm{SGRQ} \text { and } \mathrm{ACQ} \\
(p<0.05)\end{array}$ & $\begin{array}{l}\text {-Reduced PB eosinophils } \\
\text { by } 4 \mathrm{~W}\end{array}$ \\
\hline $\begin{array}{l}\text { MUSCA, } 2017 \text { Chupp } \\
\text { et al. (2017) }\end{array}$ & Phase 3b & $\begin{array}{l}\text { Severe } \\
\text { uncontrolled } \\
\text { asthma }\end{array}$ & $\begin{array}{l}\geq 0.3 \times 10^{9} \text { cells } / \mathrm{LPB} \\
\text { eosinophils or FeNO } \\
\geq 50 \text { ppb or } \mathrm{SP} \\
\text { eosinophils } \geq 3 \%\end{array}$ & $\begin{array}{l}\text { Dose: } 100 \mathrm{mg} \\
\text { Route: IV } \\
\text { Frequency: Q4W } \\
\text { Duration: } 52 \mathrm{~W}\end{array}$ & $\begin{array}{l}-58 \% \text { reduction in AAER } \\
\text { at } 24 \mathrm{~W} \\
-68 \% \text { reduction in } \\
\text { hospitalizations and } \mathrm{ED} \\
\text { visits at } 24 \mathrm{~W} \\
\text {-Improvement in SQRQ } \\
\text { score at } 4 \mathrm{~W} \\
\text {-Improved pre-BD FEV } 1 \\
\text { at } 24 \mathrm{~W}\end{array}$ & $-N / A$ \\
\hline $\begin{array}{l}\text { SIRIUS, } 2017 \text { Bel et al. } \\
\text { (2014) }\end{array}$ & Phase 3 & $\begin{array}{l}\text { Severe } \\
\text { uncontrolled } \\
\text { asthma }\end{array}$ & $\begin{array}{l}\geq 300 \text { cells/ } \mu \mathrm{l} \text { PB } \\
\text { eosinophils in previous } \\
\text { year or } \geq 150 \text { cells/ } \mu \mathrm{l} \text { PB } \\
\text { eosinophils at screening }\end{array}$ & $\begin{array}{l}\text { Dose: } 100 \text { mg } \\
\text { Route: IV } \\
\text { Frequency: Q4W } \\
\text { Duration: } 52 \mathrm{~W}\end{array}$ & $\begin{array}{l}-32 \% \text { reduction in } \\
\text { AAER } 24 \mathrm{~W} \\
-50 \% \text { reduction in OCS } \\
\text { dose at } 24 \mathrm{~W} \\
\text {-Improved } \mathrm{ACQ} \text { and } \\
\mathrm{SQRQ} \text { at } 24 \mathrm{~W} \\
\text { - No change in FEV } 1 \text { at } \\
\text { baseline or post-BD }\end{array}$ & $-N / A$ \\
\hline $\begin{array}{l}\text { COSMOS, } \\
2016 \text { Lugogo et al. } \\
(2016)\end{array}$ & Phase 3 & $\begin{array}{l}\text { Severe } \\
\text { uncontrolled } \\
\text { asthma }\end{array}$ & $\begin{array}{l}\geq 300 \text { cells/ } \mu \mathrm{l} \text { PB } \\
\text { eosinophils in previous } \\
\text { year or } \geq 150 \text { cells/ } \mu \mathrm{l} \text { PB } \\
\text { eosinophils at screening }\end{array}$ & $\begin{array}{l}\text { Dose: } 100 \mathrm{mg} \\
\text { Route: SC } \\
\text { Frequency: Q4W } \\
\text { Duration: } 52 \mathrm{~W}\end{array}$ & $\begin{array}{l}\text {-Maintained reduced } \\
\text { exacerbation rates and } \\
\text { OCS dosing }\end{array}$ & $-N / A$ \\
\hline
\end{tabular}


TABLE 2 | (Continued) Summary of randomized clinical trials assessing mepolizumab in severe asthma.

\begin{tabular}{|c|c|c|c|c|c|c|}
\hline \multicolumn{7}{|l|}{ Anti-IL-5: mepolizumab } \\
\hline $\begin{array}{l}\text { Landmark study } \\
\text { and year }\end{array}$ & RCT phase & $\begin{array}{l}\text { Asthma } \\
\text { severity }\end{array}$ & Asthma phenotype & $\begin{array}{l}\text { Dosing, duration } \\
\text { and route } \\
\text { of administration }\end{array}$ & Clinical effect & Molecular effect \\
\hline $\begin{array}{l}\text { COLUMBIA, } \\
2019 \text { Khatri et al. } \\
(2019)\end{array}$ & Phase 3 & $\begin{array}{l}\text { Severe } \\
\text { uncontrolled } \\
\text { asthma }\end{array}$ & $\begin{array}{l}\geq 300 \text { cells/uL PB } \\
\text { eosinophils in previous } \\
\text { year or } \geq 150 \text { cells/ } \mu \text { l PB } \\
\text { eosinophils at screening }\end{array}$ & $\begin{array}{l}\text { Dose: } 100 \mathrm{mg} \\
\text { Route: SC } \\
\text { Frequency: Q4W } \\
\text { Duration: } 52 \mathrm{~W}\end{array}$ & $\begin{array}{l}\text {-61\% reduction in AAER } \\
\text {-Improved ACQ-5 } \\
\text { at } 24 \mathrm{~W} \\
\text {-Improved pre-BD FEV } 1 \\
\text { at } 24 \mathrm{~W}\end{array}$ & $-N / A$ \\
\hline $\begin{array}{l}\text { COSMEX, } \\
2019 \text { Khurana et al. } \\
(2019)\end{array}$ & Phase 3b & $\begin{array}{l}\text { Severe } \\
\text { uncontrolled } \\
\text { asthma }\end{array}$ & $\begin{array}{l}\geq 300 \text { cells } / \mu \mathrm{l} \text { PB } \\
\text { eosinophils in previous } \\
\text { year or } \geq 150 \text { cells/ } \mu \mathrm{l} \text { PB } \\
\text { eosinophils at screening }\end{array}$ & $\begin{array}{l}\text { Dose: } 100 \mathrm{mg} \\
\text { Route: SC } \\
\text { Frequency: Q4W } \\
\text { Duration: } 172 \mathrm{~W}\end{array}$ & $\begin{array}{l}\text {-Maintained reduced } \\
\text { AAER and daily OCS use } \\
\text {-Improved FEV } \text { Imd }_{1} \text { and } \\
\text { ACQ-5 }\end{array}$ & -Reduced PB eosinophils \\
\hline $\begin{array}{l}\text { OSMO, } \\
2019 \text { Chapman et al. } \\
\text { (2019) }\end{array}$ & Phase 4 & $\begin{array}{l}\text { Severe } \\
\text { uncontrolled } \\
\text { asthma }\end{array}$ & $\begin{array}{l}\geq 300 \text { cells } / \mu \mathrm{l} \text { PB } \\
\text { eosinophils in previous } \\
\text { year or } \geq 150 \text { cells/ } \mu \mathrm{l} \mathrm{PB} \\
\text { eosinophils at screening }\end{array}$ & $\begin{array}{l}\text { Dose: } 100 \mathrm{mg} \\
\text { Route: SC } \\
\text { Frequency: Q4W } \\
\text { Duration: } 32 \mathrm{~W}\end{array}$ & $\begin{array}{l}-64 \% \text { reduction in AAER } \\
\text { at } 32 \mathrm{~W} \\
-69 \% \text { reduction in } \\
\text { hospitalizations and ED } \\
\text { visits at } 32 \mathrm{~W} \\
\text {-Improved } \mathrm{SGRQ} \text { and } \\
\text { pre-BD FEV }{ }_{1} \text { at } 32 \mathrm{~W}\end{array}$ & $\begin{array}{l}\text {-Reduced blood } \\
\text { eosinophils, ECP, EDN } \\
\text { at } 32 \mathrm{~W}\end{array}$ \\
\hline Bagnasco et al. (2018) & $\begin{array}{l}\text { Prospective } \\
\text { cohort study }\end{array}$ & $\begin{array}{l}\text { Severe } \\
\text { uncontrolled } \\
\text { asthma }\end{array}$ & $\begin{array}{l}\geq 300 \text { cells } / \mu \text { l PB } \\
\text { eosinophils in previous } \\
\text { year or } \geq 150 \text { cells/ } \mu \text { l PB } \\
\text { eosinophils at screening }\end{array}$ & $\begin{array}{l}12 \mathrm{M} \text { post-initiation } \\
\text { of mepolizumab }\end{array}$ & $\begin{array}{l}\text {-Reduction in OCS- } \\
\text { dependence and } \\
\text { exacerbation rate }\end{array}$ & $-N / A$ \\
\hline $\begin{array}{l}\text { REALITI-A, } \\
2020 \text { Harrison et al. } \\
(2020)\end{array}$ & $\begin{array}{l}\text { Prospective } \\
\text { cohort study }\end{array}$ & $\begin{array}{l}\text { Severe } \\
\text { uncontrolled } \\
\text { asthma }\end{array}$ & $\begin{array}{l}<300 \text { cells } / \mu \text { or } \\
\geq 300 \text { cells } / \mu \text { l PB } \\
\text { eosinophils }\end{array}$ & $\begin{array}{l}12 \mathrm{M} \text { post-initiation } \\
\text { of mepolizumab }\end{array}$ & $\begin{array}{l}\text {-Reduced AAER, } \\
\text { hospitalizations and ED } \\
\text { visits } \\
\text {-Reduced OCS } \\
\text { maintenance dose }\end{array}$ & -Reduced PB eosinophils \\
\hline $\begin{array}{l}\text { Pelaia et al. (2018a); } \\
\text { Pelaia et al. (2018b) }\end{array}$ & $\begin{array}{l}\text { Single-centered } \\
\text { observational } \\
\text { study }\end{array}$ & $\begin{array}{l}\text { Severe } \\
\text { uncontrolled } \\
\text { asthma }\end{array}$ & $\begin{array}{l}\geq 300 \text { cells } / \mu \mathrm{l} \text { PB } \\
\text { eosinophils in previous } \\
\text { year or } \geq 150 \text { cells/ } \mu \mathrm{l} \mathrm{PB} \\
\text { eosinophils at screening }\end{array}$ & $\begin{array}{l}\text { Dose: } 100 \text { mg } \\
\text { Route: SC } \\
\text { Frequency: Q4W } \\
\text { Duration: } 24 \mathrm{~W}\end{array}$ & $\begin{array}{l}\text {-Increased ACT score } \\
\text { after } 24 \mathrm{~W} \\
\text {-Improved } \mathrm{FEV}_{1} \text { and } \\
\mathrm{FEV}_{1} / \mathrm{FVC} \text { after } 24 \mathrm{~W} \\
\text {-Reduced exacerbation } \\
\text { frequency } \\
\text {-Decreased prednisone } \\
\text { dose }\end{array}$ & $\begin{array}{l}\text {-Reduced PB eosinophils } \\
\text { at } 24 \mathrm{~W}\end{array}$ \\
\hline
\end{tabular}

to treat severe eosinophilic asthmatics who were inadequately controlled on omalizumab. Interestingly, subgroup analysis demonstrated no additional benefit when both biologics were in the system, nor was there a decline seen in the benefit of omalizumab as it washed out. However, the patients who showed the most improvement in asthma control were those with eosinophilia ( $\geq 150$ cells/ $\mu$ l blood eosinophils). These findings suggest that singular targeting of IgE-dependent mechanisms may not be effective in all inflammatory subtypes of severe asthma and that perhaps targeting $\operatorname{IgE}$ is more beneficial in patients with underlying atopic status. In addition, there have been reports of $\operatorname{IgG}$ autoantibodies generated against $\operatorname{IgE}$ in allergic asthma and the formation of IgE-IgG heterocomplexes in autoimmune conditions that trigger innate immune cells (Chan et al., 2014; Henault et al., 2016). Thus, the presence of autoantibodies and immune complexes in allergic airways may impede the action of anti-IgE mAb and inadvertently induce a continued need for OCS. More studies are needed to determine what particular inflammatory profiles of severe asthma would benefit most from IgE blockade with omalizumab and if its combination with agents that target IgE-independent mechanisms would provide a synergistic effect.

\subsection{IL-5 Targeted Therapy}

Given the role of IL-5 in driving eosinophilic inflammation, it was proposed that blockade of this cytokine may attenuate T2-high inflammation. There have been three agents developed so far that target IL-5. Mepolizumab and reslizumab bind to IL-5, thereby preventing this cytokine from promoting eosinophil activation. Benralizumab, alternatively blocks the IL-5Ra, resulting in near 
TABLE 3 | Summary of randomized clinical trials assessing reslizumab in severe asthma.

Anti-IL-5: reslizumab

\begin{tabular}{|c|c|c|c|c|c|c|}
\hline $\begin{array}{l}\text { Landmark } \\
\text { study } \\
\text { and year }\end{array}$ & Study format & Asthma severity & $\begin{array}{c}\text { Asthma } \\
\text { phenotype }\end{array}$ & $\begin{array}{c}\text { Dosing, } \\
\text { duration } \\
\text { and route } \\
\text { of } \\
\text { administration }\end{array}$ & Clinical effect & Molecular effect \\
\hline $\begin{array}{l}\text { Castro et al. } \\
(2011)\end{array}$ & Phase 2 & $\begin{array}{l}\text { Severe uncontrolled } \\
\text { asthma }\end{array}$ & $\begin{array}{l}\geq 400 \text { cells } / \mu \text { PB } \\
\text { eosinophils }\end{array}$ & $\begin{array}{l}\text { Dose: } 3 \mathrm{mg} / \mathrm{kg} \\
\text { Route: IV } \\
\text { Frequency: } \\
\text { Q4W } \\
\text { Duration: } 15 \mathrm{~W}\end{array}$ & $\begin{array}{l}\text {-Improved } \mathrm{FEV}_{1} \text { but no } \\
\text { change in } \mathrm{ACQ} \text { or } \mathrm{AAER}\end{array}$ & -Reduced SP eosinophils \\
\hline $\begin{array}{l}\text { Castro et al. } \\
(2015)\end{array}$ & Phase 3 & $\begin{array}{l}\text { Severe uncontrolled } \\
\text { asthma }\end{array}$ & $\begin{array}{l}\geq 400 \text { cells } / \mu \mathrm{l} \text { PB } \\
\text { eosinophils }\end{array}$ & $\begin{array}{l}\text { Dose: } 3 \mathrm{mg} / \mathrm{kg} \\
\text { Route: IV } \\
\text { Frequency: } \\
\text { Q4W } \\
\text { Duration: } 52 \mathrm{~W}\end{array}$ & -Reduced AAER & $-\mathrm{N} / \mathrm{A}$ \\
\hline $\begin{array}{l}\text { Bjermer et al. } \\
(2016)\end{array}$ & Phase 3 & $\begin{array}{l}\text { Severe uncontrolled } \\
\text { asthma }\end{array}$ & $\begin{array}{l}\geq 400 \text { cells } / \mu \text { PB } \\
\text { eosinophils }\end{array}$ & $\begin{array}{l}\text { Dose: } 3 \mathrm{mg} / \mathrm{kg} \\
\text { Route: IV } \\
\text { Frequency: } \\
\text { Q4W } \\
\text { Duration: } 16 \mathrm{~W}\end{array}$ & $\begin{array}{l}\text {-Improved ACQ, AQLQ, FEV } 1 \\
\text { and FVC }\end{array}$ & $-N / A$ \\
\hline $\begin{array}{l}\text { Corren et al. } \\
(2016)\end{array}$ & Phase 3 & $\begin{array}{l}\text { Severe uncontrolled } \\
\text { asthma }\end{array}$ & $\begin{array}{l}\geq 400 \text { or } \\
<400 \text { cells/ } \mu \text { PB } \\
\text { eosinophils }\end{array}$ & $\begin{array}{l}\text { Dose: } 3 \mathrm{mg} / \mathrm{kg} \\
\text { Route: IV } \\
\text { Frequency: } \\
\text { Q4W } \\
\text { Duration: } 16 \mathrm{~W}\end{array}$ & $\begin{array}{l}\text {-No change in mean } \mathrm{FEV}_{1} \text {, } \\
\text { except in subgroup analysis } \\
\text { with eosinophilia }\end{array}$ & -Reduced PB eosinophils \\
\hline $\begin{array}{l}\text { Murphy et al. } \\
(2017)\end{array}$ & Phase 3 & $\begin{array}{l}\text { Severe uncontrolled } \\
\text { asthma }\end{array}$ & $\begin{array}{l}\geq 400 \text { cells/ } / \mathrm{ll} \mathrm{PB} \\
\text { eosinophils }\end{array}$ & $\begin{array}{l}\text { Dose: } 3 \mathrm{mg} / \mathrm{kg} \\
\text { Route: IV } \\
\text { Frequency: } \\
\text { Q4W } \\
\text { Duration: } 24 \mathrm{M}\end{array}$ & $\begin{array}{l}\text {-Improved ACQ, AQLQ, FEV } 1 \\
\text { and FVC }\end{array}$ & -Reduced PB eosinophils \\
\hline $\begin{array}{l}\text { Brusselle et al. } \\
\text { (2017) }\end{array}$ & Phase 3 & $\begin{array}{l}\text { Severe uncontrolled } \\
\text { asthma }\end{array}$ & $\begin{array}{l}\geq 400 \text { cells } / \mu \mathrm{l} \text { PB } \\
\text { eosinophils }\end{array}$ & $\begin{array}{l}\text { Dose: } 3 \text { mg/kg } \\
\text { Route: IV } \\
\text { Frequency: } \\
\text { Q4W } \\
\text { Duration: } 52 \text { W }\end{array}$ & $\begin{array}{l}\text {-Reduced AAER over } 52 \mathrm{~W} \text {, } \\
\text { and exacerbations requiring } \\
\text { hospitalization/ED visits } \\
\text {-Improved ACQ and AQLQ for } \\
\text { late onset patients }\end{array}$ & $-N / A$ \\
\hline $\begin{array}{l}\text { Weinstein et al. } \\
\text { (2019) }\end{array}$ & Phase 3 & $\begin{array}{l}\text { Severe uncontrolled } \\
\text { asthma with CRS }\end{array}$ & $\begin{array}{l}\geq 400 \text { cells/ } / \mu \mathrm{PB} \\
\text { eosinophils }\end{array}$ & $\begin{array}{l}\text { Dose: } 3 \mathrm{mg} / \mathrm{kg} \\
\text { Route: IV } \\
\text { Frequency: } \\
\text { Q4W } \\
\text { Duration: } 52 \mathrm{~W}\end{array}$ & $\begin{array}{l}\text {-Reduced AAER } \\
\text {-Improved FEV }\end{array}$ & $-N / A$ \\
\hline $\begin{array}{l}\text { Bernstein et al. } \\
(2020)\end{array}$ & Phase 3 & $\begin{array}{l}\text { Severe uncontrolled } \\
\text { asthma }\end{array}$ & $\begin{array}{l}\geq 300 \text { cells } / \mu \text { PB } \\
\text { eosinophils }\end{array}$ & $\begin{array}{l}\text { Dose: } 110 \text { mg } \\
\text { Route: SC } \\
\text { Frequency: } \\
\text { Q4W } \\
\text { Duration: } 52 \text { W }\end{array}$ & $\begin{array}{l}\text {-No difference in AAER, } \\
\text { except in those with PB } \\
\text { eosinophils } \geq 400 \\
\text {-No difference in steroid } \\
\text { dosing }\end{array}$ & $-N / A$ \\
\hline
\end{tabular}

(Continued on following page) 
TABLE 3 | (Continued) Summary of randomized clinical trials assessing reslizumab in severe asthma.

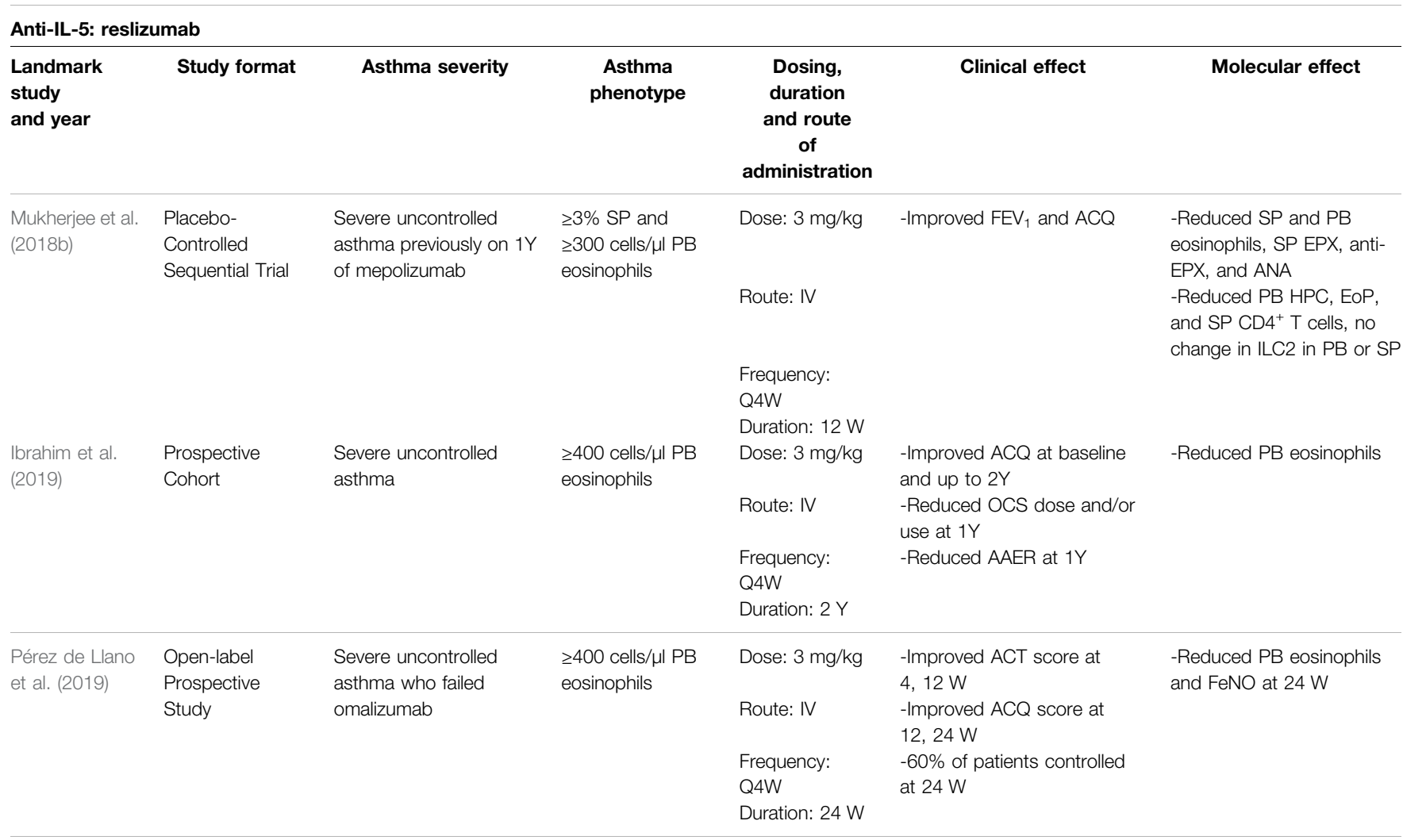

complete depletion of peripheral eosinophils through antibodydependent cell-mediated cytotoxicity (ADCC) involving NK cells.

The story of mepolizumab provides a valuable lesson for individualized asthma management. Leckie et al. first showed in 2000 that despite mepolizumab resulting in attenuation of blood and sputum eosinophils, there was a lack of translation into meaningful clinical outcomes in patients with asthma (Leckie et al., 2000). Large RCTs later followed, which also failed to show clinical efficacy, and as a result the development of anti-IL-5, or indeed any anti-eosinophil therapy, was tabled for many years (Flood-Page et al., 2003). In fact, the overall importance of IL-5 and eosinophils in asthma was brought into question. It should be noted that these initial trials did not select appropriate patient populations based on eosinophilia and a T2-high profile, but rather selected a heterogeneous pool of asthmatic patients with a variety of immunological profiles. Hence, it is not surprising that there was a lack of clinical response to anti-IL-5 treatment in these early studies.

This led to a pivotal change in studies examining anti-IL-5 therapy through the specific targeting of T2-high patients. Using patient selection criteria for the T2-high profile, Haldar et al. reported that mepolizumab treatment in severe asthmatics that specifically exhibited eosinophilia ( $\geq 3 \%$ sputum eosinophils in last 12 months), not only reduced blood and sputum eosinophils but also resulted in 43\% fewer exacerbations (Haldar et al., 2009). Further, Nair et al. showed that mepolizumab administration to severe prednisone-dependent eosinophilic asthmatics resulted in
OCS tapering, where patients had an $83 \%$ reduction of their maximum prednisone dose versus $47 \%$ with placebo. They also reported fewer exacerbations, improved asthma control, and increased $\mathrm{FEV}_{1}$ with an associated decrease in sputum and blood eosinophils (Nair et al., 2009). Multiple other RCTs and real-world investigations have shown that mepolizumab has a corticosteroid-sparing effect in this population, with reductions in annual asthma exacerbation rates (AAER) by 39-52\%, improvement in lung function, and asthma symptom scores, as well as improvement in overall health-related quality of life (Table 2) (Pavord et al., 2012; Bel et al., 2014; Chupp et al., 2017; Pelaia et al., 2018b; Harrison et al., 2020).

Three important observations should be noted from the mepolizumab data. Firstly, the efficacy of mepolizumab is based on patients having baseline eosinophilia, and as such, we must identify individuals that will benefit from this treatment in the first place. Peripheral blood eosinophils were initially chosen as a biomarker in the mepolizumab studies for a few reasons. Peripheral eosinophils have been identified through cluster analyses to predict responsiveness to mepolizumab (Ortega et al., 2016). Indeed, it is one of the most simple and practical ways to identify Th2 inflammation and as such, patients that may benefit from anti-IL-5 therapy. The caveat to this is that peripheral eosinophils can be highly variable and a single measurement of peripheral blood eosinophils may not reflect the average level of cells throughout an extended period of time. Thus, the use of peripheral blood eosinophils to guide therapy to anti-IL-5 biologics may not be as ideal as sputum eosinophils. 
TABLE 4 | Summary of randomized clinical trials assessing benralizumab in severe asthma.

Anti-IL-5R alpha: benralizumab

\begin{tabular}{|c|c|c|c|c|c|c|}
\hline $\begin{array}{l}\text { Landmark study } \\
\text { and year }\end{array}$ & $\begin{array}{l}\text { Study } \\
\text { format }\end{array}$ & $\begin{array}{l}\text { Asthma } \\
\text { severity }\end{array}$ & Asthma phenotype & $\begin{array}{l}\text { Dosing, duration } \\
\text { and route of } \\
\text { administration }\end{array}$ & Clinical effect & Molecular effect \\
\hline Nowak et al. (2015) & Phase 2 & $\begin{array}{l}\text { Severe } \\
\text { uncontrolled } \\
\text { asthma }\end{array}$ & $\begin{array}{l}<450 \text { cells } / \mu \text { or } \\
\geq 450 \text { cells } / \mu \text { PB } \\
\text { eosinophils }\end{array}$ & $\begin{array}{l}\text { Dose: } 0.3 \mathrm{mg} / \mathrm{kg} \\
\text { or } 1 \mathrm{mg} / \mathrm{kg} \\
\text { Route: SC } \\
\text { Frequency: Once } \\
\text { Duration: } 24 \mathrm{~W}\end{array}$ & $\begin{array}{l}-49 \% \text { reduction in } \\
\text { AAER } \\
\text {-60\% reduction in } \\
\text { hospitalization } \\
\text {-No change in } \mathrm{FEV}_{1} \text {, } \\
\text { ACQ, AQLQ }\end{array}$ & $\begin{array}{l}\text {-Reduced PB eosinophils up } \\
\text { to } 12 \mathrm{~W} \\
\text {-Reduced ECP and EDN }\end{array}$ \\
\hline $\begin{array}{l}\text { CALIMA, } \\
2016 \text { FitzGerald et al. } \\
(2016)\end{array}$ & Phase 3 & $\begin{array}{l}\text { Severe } \\
\text { uncontrolled } \\
\text { asthma }\end{array}$ & $\begin{array}{l}<300 \text { cells } / \mu l \text { or } \\
\geq 300 \text { cells } / \mu \text { PB } \\
\text { eosinophils }\end{array}$ & $\begin{array}{l}\text { Dose: } 30 \text { mg } \\
\text { Route: SC } \\
\text { Frequency: } \\
\text { Q4W, Q8W } \\
\text { Duration: } 56 \text { W }\end{array}$ & $\begin{array}{l}-28 \% \text { reduction in } \\
\mathrm{AAER} \text { at } 56 \mathrm{~W} \\
\text {-Improved } \mathrm{ACQ} \\
\mathrm{AQLQ} \text {, pre-BD FEV }\end{array}$ & -Reduced PB eosinophils \\
\hline $\begin{array}{l}\text { SIROCCO, } \\
2016 \text { Bleecker et al. } \\
\text { (2016) }\end{array}$ & Phase 3 & $\begin{array}{l}\text { Severe } \\
\text { uncontrolled } \\
\text { asthma }\end{array}$ & $\begin{array}{l}<300 \text { cells } / \mu \text { or } \\
\geq 300 \text { cells } / \mu \text { PB } \\
\text { eosinophils }\end{array}$ & $\begin{array}{l}\text { Dose: } 30 \text { mg } \\
\text { Route: SC } \\
\text { Frequency: } \\
\text { Q4W, Q8W } \\
\text { Duration: } 48 \text { W }\end{array}$ & $\begin{array}{l}\text {-Reduced AAER, } \\
\text { regardless of } \\
\text { eosinophils at } 48 \mathrm{~W} \\
\text {-Improved } \mathrm{FEV}_{1}, \mathrm{ACQ} \text { - } \\
6, \mathrm{AQLQ} \text { at } 48 \mathrm{~W}\end{array}$ & $\begin{array}{l}\text {-Reduced PB eosinophils } \\
\text { by } 4 \mathrm{~W}\end{array}$ \\
\hline $\begin{array}{l}\text { ZONDA, } 2017 \text { Nair et al. } \\
(2017)\end{array}$ & Phase 3 & $\begin{array}{l}\text { Severe } \\
\text { uncontrolled } \\
\text { asthma }\end{array}$ & $\begin{array}{l}\geq 150 \text { cells } / \mu \mathrm{l} \mathrm{PB} \\
\text { eosinophils }\end{array}$ & $\begin{array}{l}\text { Dose: } 30 \text { mg } \\
\text { Route: SC } \\
\text { Frequency: } \\
\text { Q4W, Q8W } \\
\text { Duration: } 28 \text { W }\end{array}$ & $\begin{array}{l}\text {-75\% reduction in OCS } \\
\text { dose } \\
\text {-Improved AAER, } \\
\text { ACQ-6, AQLQ } \\
\text {-No effect on FEV } 1\end{array}$ & $-N / A$ \\
\hline $\begin{array}{l}\text { BISE, } 2017 \text { Ferguson } \\
\text { et al. (2017) }\end{array}$ & Phase 3 & $\begin{array}{l}\text { Mild-moderate } \\
\text { persistent } \\
\text { asthma }\end{array}$ & $\begin{array}{l}<300 \text { cells } / \mu \text { or } \\
\geq 300 \text { cells } / \mu \text { PB } \\
\text { eosinophils }\end{array}$ & $\begin{array}{l}\text { Dose: } 30 \mathrm{mg} \\
\text { Route: SC } \\
\text { Frequency: Q4W } \\
\text { Duration: } 12 \mathrm{~W}\end{array}$ & $\begin{array}{l}\text {-Increased pre-BD } \\
\mathrm{FEV}_{1} \text { at } 12 \mathrm{~W}\end{array}$ & $-N / A$ \\
\hline Chupp et al. (2019) & Phase 3 & $\begin{array}{l}\text { Severe } \\
\text { uncontrolled } \\
\text { asthma }\end{array}$ & $\begin{array}{l}\geq 300 \text { cells } / \mu \mathrm{l} \text { PB } \\
\text { eosinophils }\end{array}$ & $\begin{array}{l}\text { Dose: } 30 \text { mg } \\
\text { Route: SC } \\
\text { Frequency: } \\
\text { Q4W, Q8W } \\
\text { Duration: } \\
\text { 28-56 W }\end{array}$ & $\begin{array}{l}\text {-Improvement in } \\
\text { morning PEF from } \\
\text { baseline within } 2 \mathrm{~W}\end{array}$ & $-N / A$ \\
\hline $\begin{array}{l}\text { BORA, } 2019 \text { Busse } \\
\text { et al. (2019) }\end{array}$ & Phase 3 & $\begin{array}{l}\text { Severe } \\
\text { uncontrolled } \\
\text { asthma }\end{array}$ & $\begin{array}{l}<300 \text { cells } / \mu \text { or } \\
\geq 300 \text { cells } / \mu \text { PB } \\
\text { eosinophils }\end{array}$ & $\begin{array}{l}\text { Dose: } 30 \text { mg } \\
\text { Route: SC } \\
\text { Frequency: } \\
\text { Q4W Q8W } \\
\text { Duration: } 56 \text { W }\end{array}$ & $\begin{array}{l}\text {-72\% of patients with } \\
\text { eosinophilia did not } \\
\text { have exacerbation } \\
\text {-Maintained } \\
\text { improvement in FEV } 1 \\
\text { and } A C Q, A Q L Q\end{array}$ & $-N / A$ \\
\hline
\end{tabular}

(Continued on following page) 
TABLE 4 | (Continued) Summary of randomized clinical trials assessing benralizumab in severe asthma.

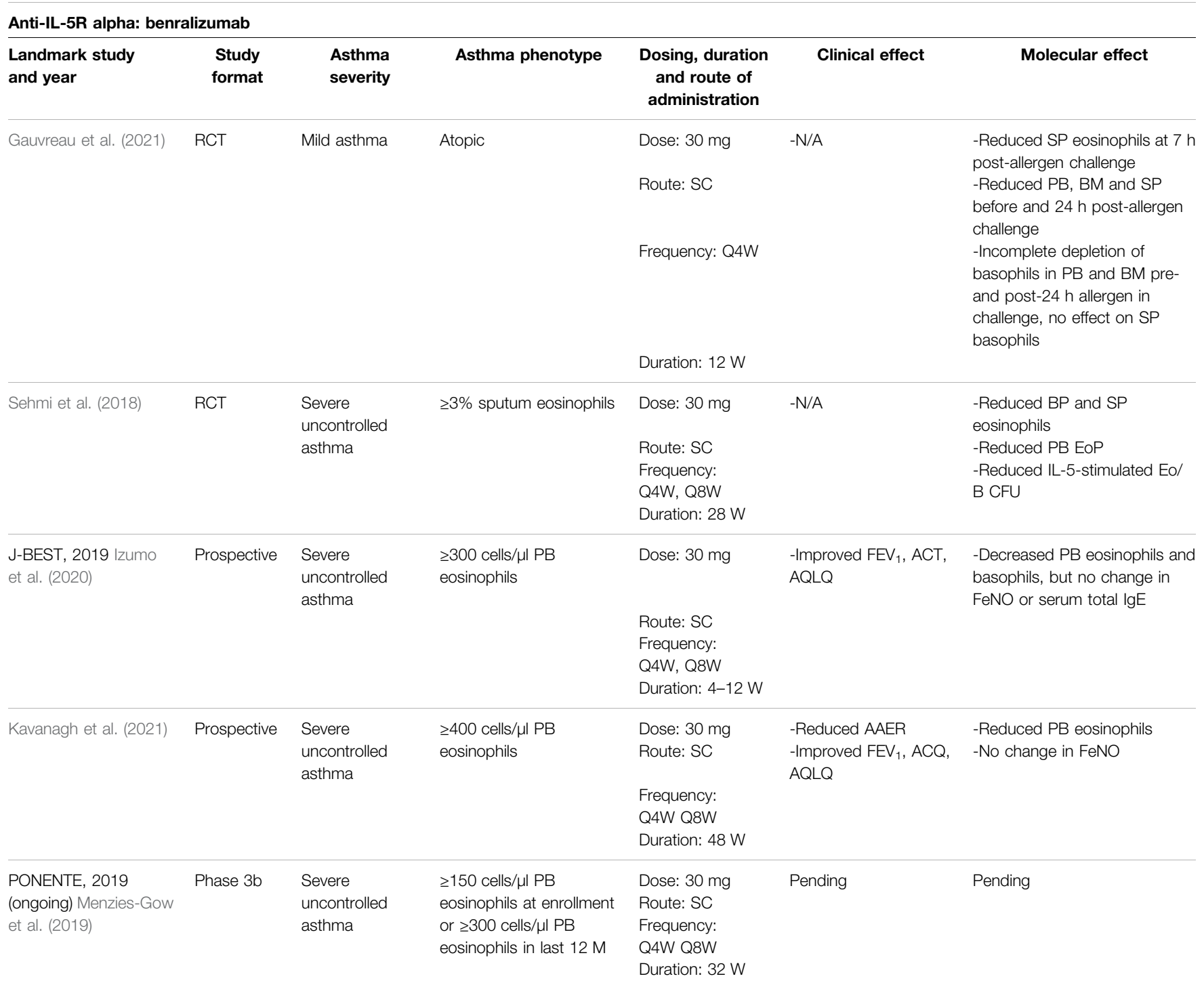

Unfortunately, there is a lack of global availability of sputum labs making it difficult to use sputum eosinophils as a universal biomarker. However, if available, sputum counts can be reproducible and reliable if the proper processing technique is done. We propose that peripheral eosinophilia may be helpful to predict response to anti-IL-5 agents but has a limited role in monitoring response to treatment. As previously reported, in 250 patients with baseline blood eosinophilia ( $\geq 400$ cells/ $\mu$ l) treated with either mepolizumab or reslizumab for at least 4 months, there was an overall suboptimal response in 43\% (Mukherjee et al., 2018b). Of the 129 patients in whom paired blood and sputum eosinophils were available 4 months post-treatment, there were 65 suboptimal responders, $78 \%$ of who had sputum eosinophils $\geq 3 \%$. Only seven of these patients had blood eosinophils $\geq 400 \mathrm{cells} / \mu \mathrm{l}$. As such, there is a discordance between the two compartments, which may be, in part, due to in situ eosinophilopoiesis. As such, the use of sputum eosinophils to monitor response to treatment may be more reliable than peripheral eosinophils. Secondly, despite multiple studies showing clinical benefit, the effect of mepolizumab was ultimately incomplete, with $\sim 50 \%$ reduction in exacerbation rates despite ablation of peripheral eosinophilia. This begs the question as to whether peripheral blood eosinophils should be the only biomarker to determine whether patients would benefit from this biologic. Lastly, and most importantly, we need to re-examine the spotlight on eosinophils and their overall importance in T2high inflammation. Aside from eosinophils, there are cytokines and effector cells, which may be equally, if not more important than eosinophils, and as such, should be targeted.

Reslizumab was the second anti-IL-5 biologic to be brought to market. In contrast to mepolizumab, it is administered intravenously with weight-based dosing. A phase 2 RCT showed that reslizumab reduced both sputum and blood eosinophils, with an associated improvement in $\mathrm{FEV}_{1}$ (Kips 
TABLE 5 | Summary of randomized clinical trials assessing IL-4/13 in severe asthma.

\begin{tabular}{|c|c|c|c|c|c|c|}
\hline \multicolumn{7}{|l|}{ Anti-IL-4/13 } \\
\hline $\begin{array}{l}\text { Landmark study } \\
\text { and year }\end{array}$ & $\begin{array}{c}\text { RCT } \\
\text { phase }\end{array}$ & Asthma severity & Inflammatory profile & $\begin{array}{l}\text { Dosing, duration } \\
\text { and route } \\
\text { of administration }\end{array}$ & Clinical effect & Molecular effect \\
\hline Hodsman et al. (2013) & Phase 1 & Mild asthma & $\begin{array}{l}\text { Independent of PB } \\
\text { eosinophils }\end{array}$ & $\begin{array}{l}\text { Biologic: } \\
\text { GSK679586 } \\
\text { Dosing: } \\
0.005-10 \mathrm{mg} / \mathrm{kg} \\
\text { Frequency: Q4W } \\
\text { Route: IV } \\
\text { Duration: } 28 \mathrm{~W}\end{array}$ & $-N / A$ & $\begin{array}{l}\text {-Increased serum IL-13 } \\
\text {-Reduced FeNO at 2W } \\
\text { and } 8 \mathrm{~W}\end{array}$ \\
\hline De Boever et al. (2014) & Phase 2 & $\begin{array}{l}\text { Severe uncontrolled } \\
\text { asthma }\end{array}$ & $\begin{array}{l}\geq 140 \text { cells ul PB } \\
\text { eosinophils }\end{array}$ & $\begin{array}{l}\text { Biologic: } \\
\text { GSK679586 } \\
\text { Dosing: } 10 \mathrm{mg} / \mathrm{kg} \\
\text { Frequency: Q4W } \\
\text { Route: IV } \\
\text { Duration: } 24 \mathrm{~W}\end{array}$ & $\begin{array}{l}\text {-No change in ACQ score, } \\
\text { FEV }_{1}, \text { AAER }\end{array}$ & $\begin{array}{l}\text {-No difference in serum IL- } \\
13 \text { or lgE } \\
\text {-No difference in PB } \\
\text { eosinophils }\end{array}$ \\
\hline Piper et al. (2013) & $\begin{array}{l}\text { Phase } \\
2 \mathrm{a}\end{array}$ & $\begin{array}{l}\text { Moderate-severe } \\
\text { uncontrolled asthma }\end{array}$ & $\begin{array}{l}\text { Independent of PB } \\
\text { eosinophils }\end{array}$ & $\begin{array}{l}\text { Biologic: } \\
\text { Tralokinumab } \\
\text { Dosing: } \\
\text { 150-600 mg } \\
\text { Frequency: Q2W } \\
\text { Route: SC } \\
\text { Duration: } 24 \mathrm{~W}\end{array}$ & $\begin{array}{l}\text {-No change in ACQ, pre-BD } \\
\text { FEV }_{1} \text {, FVC, PEF, AAER. }\end{array}$ & $\mathrm{N} / \mathrm{A}$ \\
\hline Russell et al. (2018) & Phase 2 & $\begin{array}{l}\text { Moderate-severe } \\
\text { uncontrolled asthma }\end{array}$ & $\begin{array}{l}\text { Independent of PB } \\
\text { eosinophils }\end{array}$ & $\begin{array}{l}\text { Biologic: } \\
\text { Tralokinumab } \\
\text { Dosing: } 300 \mathrm{mg} \\
\text { Frequency: Q2W } \\
\text { Route: SC } \\
\text { Duration: } 12 \mathrm{~W}\end{array}$ & $-N / A$ & $\begin{array}{l}\text {-No change in bronchial } \\
\text { eosinophils at } 12 \mathrm{~W} \\
\text { - No change in PB or SP } \\
\text { eosinophils or serum IgE }\end{array}$ \\
\hline $\begin{array}{l}\text { STRATOS I, II, 2018; } \\
\text { Panettieri et al. (2018) }\end{array}$ & Phase 3 & $\begin{array}{l}\text { Severe uncontrolled } \\
\text { asthma }\end{array}$ & $\begin{array}{l}\geq 37 \mathrm{ppb} \text { FeNO or } \\
<37 \mathrm{ppb}\end{array}$ & $\begin{array}{l}\text { Biologic: } \\
\text { Tralokinumab } \\
\text { Dosing: } 300 \mathrm{mg} \\
\text { Frequency: } \\
\text { Q2W, Q4W } \\
\text { Route: SC } \\
\text { Duration: } 52 \mathrm{~W}\end{array}$ & $\begin{array}{l}\text {-Reduced AAER at } 2 \mathrm{~W} \text { in } \\
\text { FeNO-high patients }\end{array}$ & $-N / A$ \\
\hline Corren et al. (2011) & Phase 2 & $\begin{array}{l}\text { Severe uncontrolled } \\
\text { asthma }\end{array}$ & $\begin{array}{l}\text { Periostin } \geq 50 \text { or } \\
<50 \mathrm{ng} / \mathrm{ml}\end{array}$ & $\begin{array}{l}\text { Biologic: } \\
\text { Lebrikizumab } \\
\text { Dose: } 250 \mathrm{mg} \\
\text { Frequency: Q4W } \\
\text { Route: SC } \\
\text { Duration: } 24 \mathrm{~W}\end{array}$ & $\begin{array}{l}-60 \% \text { reduction in } \\
\text { exacerbation at } 24 \mathrm{~W} \\
\text {-Improved } \mathrm{FEV}_{1} \text {, at } 12 \mathrm{~W} \\
\text {-No change in } \mathrm{ACQ}\end{array}$ & $\begin{array}{l}-19 \% \text { reduction in FeNO } \\
\text { at } 12 \mathrm{~W} \\
\text {-Decreased CCL13, } \\
\mathrm{CCL} 17, \text { total IgE levels } \\
\text { at } 24 \mathrm{~W}\end{array}$ \\
\hline Noonan et al. (2013) & Phase 2 & Mild asthma & $\begin{array}{l}\text { Periostin } \geq 50 \text { or } \\
<50 \mathrm{ng} / \mathrm{ml}\end{array}$ & $\begin{array}{l}\text { Biologic: } \\
\text { Lebrikizumab } \\
\text { Dose: 125-500 mg } \\
\text { Frequency: Q4W } \\
\text { Route: SC } \\
\text { Duration: } 12 \mathrm{~W}\end{array}$ & $\begin{array}{l}\text {-No change in } \mathrm{FEV}_{1} \text {, pre-PB } \\
\text { PEF, AQLQ }\end{array}$ & $-N / A$ \\
\hline $\begin{array}{l}\text { LUTE, VERSE, } \\
2015 \text { Hanania et al. } \\
\text { (2015) }\end{array}$ & Phase 3 & $\begin{array}{l}\text { Severe uncontrolled } \\
\text { asthma }\end{array}$ & $\begin{array}{l}\text { Periostin } \geq 50 \text { or } \\
<50 \mathrm{ng} / \mathrm{ml}\end{array}$ & $\begin{array}{l}\text { Biologic: } \\
\text { Lebrikizumab } \\
\text { Dose: 37.5-250 mg } \\
\text { Frequency: Q4W } \\
\text { Route: SC } \\
\text { Duration: } 52 \mathrm{~W}\end{array}$ & $\begin{array}{l}\text {-60\% reduction in } \\
\text { exacerbation in periostin- } \\
\text { high patients } \\
\text {-No dose response for } \\
\text { exacerbation } \\
\text {-Improved } \mathrm{FEV}_{1} \text { at } 12 \mathrm{~W}\end{array}$ & $\begin{array}{l}\text { - Reduction in PB } \\
\text { eosinophils and FeNO }\end{array}$ \\
\hline
\end{tabular}


TABLE 5 | (Continued) Summary of randomized clinical trials assessing IL-4/13 in severe asthma.

\begin{tabular}{|c|c|c|c|c|c|c|}
\hline \multicolumn{7}{|l|}{ Anti-IL-4/13 } \\
\hline $\begin{array}{l}\text { Landmark study } \\
\text { and year }\end{array}$ & $\begin{array}{c}\text { RCT } \\
\text { phase }\end{array}$ & Asthma severity & Inflammatory profile & $\begin{array}{l}\text { Dosing, duration } \\
\text { and route } \\
\text { of administration }\end{array}$ & Clinical effect & Molecular effect \\
\hline $\begin{array}{l}\text { LAVOLTA I, II, 2016; } \\
\text { Hanania et al. (2016) }\end{array}$ & Phase 2 & $\begin{array}{l}\text { Severe uncontrolled } \\
\text { asthma }\end{array}$ & $\begin{array}{l}\geq 300 \text { cells } \mu \mathrm{l} \mathrm{PB} \\
\text { eosinophils or periostin } \\
\geq 50 \mathrm{ng} / \mathrm{ml}\end{array}$ & $\begin{array}{l}\text { Biologic: } \\
\text { Lebrikizumab } \\
\text { Dose: } 37.5-125 \text { mg } \\
\text { Frequency: Q4W } \\
\text { Route: SC } \\
\text { Duration: } 52 \mathrm{~W}\end{array}$ & $\begin{array}{l}-70 \% \text { reduction in } \\
\text { exacerbation in periostin- } \\
\text { high patients }\end{array}$ & $-N / A$ \\
\hline $\begin{array}{l}\text { STRETTO, 2018; } \\
\text { Korenblat et al. (2018) }\end{array}$ & Phase 3 & $\begin{array}{l}\text { Mild-moderate } \\
\text { asthma }\end{array}$ & $\begin{array}{l}\geq 300 \text { cells } \mu \mathrm{l} \mathrm{PB} \\
\text { eosinophils or periostin } \\
\geq 50 \mathrm{ng} / \mathrm{ml}\end{array}$ & $\begin{array}{l}\text { Biologic: } \\
\text { Lebrikizumab } \\
\text { Dose: } 125 \mathrm{mg} \\
\text { Frequency: Q4W } \\
\text { Route: SC } \\
\text { Duration: } 12 \mathrm{~W}\end{array}$ & $\begin{array}{l}\text {-No change in } \mathrm{FEV}_{1} \text {, pre-PB } \\
\text { PEF, AQLQ }\end{array}$ & $-N / A$ \\
\hline Wenzel et al. (2007) & $\begin{array}{l}\text { Phase } \\
2 \mathrm{a}\end{array}$ & Atopic asthma & $\begin{array}{l}\text { Independent of PB } \\
\text { eosinophils }\end{array}$ & $\begin{array}{l}\text { Biologic: Pitrakinra } \\
\text { Dose: } 25,60 \mathrm{mg} \\
\text { Frequency: OD, BID } \\
\text { Route: SC, Inhaled } \\
\text { Duration: } 12 \mathrm{~W}\end{array}$ & $\begin{array}{l}\text {-No change in } \mathrm{FEV}_{1} \text { for } \mathrm{SC} \\
\text { trial but reduction with inhaled }\end{array}$ & $\begin{array}{l}\text { - Decreased FeNO with } \\
\text { inhaled group } \\
\text { - No change in SP or PB } \\
\text { eosinophils } \\
\text { - No change in serum lgE }\end{array}$ \\
\hline Otulana et al. (2011) & $\begin{array}{l}\text { Phase } \\
2 b\end{array}$ & $\begin{array}{l}\text { Moderate-severe } \\
\text { uncontrolled asthma }\end{array}$ & $\begin{array}{l}\text { Independent of blood } \\
\text { eosinophils or atopic } \\
\text { status }\end{array}$ & $\begin{array}{l}\text { Biologic: Pitrakinra } \\
\text { Dose: 1-10 mg BID } \\
\text { Route: Inhaled } \\
\text { Duration: } 12 \mathrm{~W}\end{array}$ & $\begin{array}{l}\text {-Reduced exacerbation in } \\
\text { eosinophilic group } \\
\text {-Improvement in symptom } \\
\text { scores and spirometry }\end{array}$ & $-N / A$ \\
\hline Corren et al. (2010) & Phase 2 & $\begin{array}{l}\text { Moderate-severe } \\
\text { asthma }\end{array}$ & $\begin{array}{l}\text { Independent of blood } \\
\text { eosinophils or atopic } \\
\text { status }\end{array}$ & $\begin{array}{l}\text { Biologic: AMG } 317 \\
\text { Dose: } 75-300 \text { mg } \\
\text { Frequency: Q4W } \\
\text { Route: SC } \\
\text { Duration: } 12 \mathrm{~W}\end{array}$ & $\begin{array}{l}\text {-No improvement in ACQ } \\
\text {-No decrease in exacerbation }\end{array}$ & $\begin{array}{l}\text { - No change in serum lgE } \\
\text { - No change in sputum } \\
\text { eosinophils, FeNO }\end{array}$ \\
\hline Wenzel et al. (2013) & Phase 2 & $\begin{array}{l}\text { Moderate-severe } \\
\text { asthma }\end{array}$ & $\begin{array}{l}\geq 300 \text { cells } \mu \mathrm{l} \text { PB } \\
\text { eosinophils or SP } \\
\text { eosinophils } \geq 3 \%\end{array}$ & $\begin{array}{l}\text { Biologic: Dupilumab } \\
\text { Dose: } 300 \text { mg } \\
\text { Frequency: Q1W } \\
\text { Route: SC } \\
\text { Duration: } 12 \mathrm{~W}\end{array}$ & $\begin{array}{l}-87 \% \text { reduction in } \\
\text { exacerbation at } 12 \mathrm{~W} \\
\text {-Increase in } \mathrm{FEV}_{1} \text { predicted } \\
\text { from } 2 \text { to } 12 \mathrm{~W} \\
\text {-Improved } \mathrm{ACQ} \text { at } 3 \mathrm{~W}\end{array}$ & $\begin{array}{l}\text { - Reduced FeNO from } 4 \\
\text { to } 12 \mathrm{~W} \\
\text { - Decrease in serum TARC, } \\
\text { eotaxin-3 or lgE } \\
\text { - No change in PB or SP } \\
\text { eosinophils }\end{array}$ \\
\hline Wenzel et al. (2016) & $\begin{array}{l}\text { Phase } \\
2 \mathrm{~b}\end{array}$ & $\begin{array}{l}\text { Severe uncontrolled } \\
\text { asthma }\end{array}$ & $\begin{array}{l}<300 \text { cells/ul or } \\
\geq 300 \text { cells/ } \mu \text { PB } \\
\text { eosinophils }\end{array}$ & $\begin{array}{l}\text { Biologic: Dupilumab } \\
\text { Dose: 200, } 300 \text { mg } \\
\text { Frequency: Q2-4W } \\
\text { Route: SC } \\
\text { Duration: } 24 \mathrm{~W}\end{array}$ & $\begin{array}{l}\text {-Increased } \mathrm{FEV}_{1} \text { in those } \\
\text { with } \mathrm{PB} \geq 300 \text { cells/uL } \\
\text { eosinophils } \\
\text {-Reduced } \mathrm{AAER} \\
\text {-Improved } \mathrm{ACQ} \text {, regardless } \\
\text { of eosinophils }\end{array}$ & -Reduced FeNO at $24 \mathrm{~W}$ \\
\hline $\begin{array}{l}\text { LIBERTY ASTHMA } \\
\text { QUEST, 2018; Castro } \\
\text { et al. (2018) }\end{array}$ & Phase 3 & $\begin{array}{l}\text { Severe uncontrolled } \\
\text { asthma }\end{array}$ & $\begin{array}{l}<300 \text { cells } / \mu \text { or } \\
\geq 300 \text { cells } / \mu \text { PB } \\
\text { eosinophils }\end{array}$ & $\begin{array}{l}\text { Biologic: Dupilumab } \\
\text { Dose: 200, } 300 \text { mg } \\
\text { Frequency: Q2W } \\
\text { Route: SC } \\
\text { Duration: } 52 \mathrm{~W}\end{array}$ & $\begin{array}{l}\text {-Reduced AAER } \\
\text {-Increased FEV } 1 \text { at } 12 \mathrm{~W} \\
\text {-Improved ACQ, AQLQ scores }\end{array}$ & $\begin{array}{l}\text {-Reduced FeNO, serum } \\
\text { IgE, periostin, eotaxin-3, } \\
\text { TARC at } 52 \mathrm{~W} \\
\text {-Transient increased PB } \\
\text { eosinophils with } \\
\text { increased ECP }\end{array}$ \\
\hline
\end{tabular}


TABLE 5 | (Continued) Summary of randomized clinical trials assessing IL-4/13 in severe asthma.

\begin{tabular}{|c|c|c|c|c|c|c|}
\hline \multicolumn{7}{|l|}{ Anti-IL-4/13 } \\
\hline $\begin{array}{l}\text { Landmark study } \\
\text { and year }\end{array}$ & $\begin{array}{c}\text { RCT } \\
\text { phase }\end{array}$ & Asthma severity & Inflammatory profile & $\begin{array}{l}\text { Dosing, duration } \\
\text { and route } \\
\text { of administration }\end{array}$ & Clinical effect & Molecular effect \\
\hline $\begin{array}{l}\text { LIBERTY ASTHMA } \\
\text { QUEST, 2018; Castro } \\
\text { et al. (2018) }\end{array}$ & Phase 3 & $\begin{array}{l}\text { Severe uncontrolled } \\
\text { asthma }\end{array}$ & $\begin{array}{l}<300 \text { cells } / \mu \text { or } \\
\geq 300 \text { cells } / \mu \text { PB } \\
\text { eosinophils }\end{array}$ & $\begin{array}{l}\text { Biologic: Dupilumab } \\
\text { Dose: } 300 \mathrm{mg} \\
\text { Frequency: Q2W } \\
\text { Route: SC } \\
\text { Duration: } 24 \mathrm{~W}\end{array}$ & $\begin{array}{l}\text {-Reduced OCS dose } \\
\text {-Reduced rate of severe } \\
\text { asthma exacerbations and } \\
\text { AAER } \\
\text {-Increased FEV } 1 \\
\text {-Improved ACQ }\end{array}$ & $\begin{array}{l}\text {-Reduced FeNO } \\
\text {-Transient increased PB } \\
\text { eosinophils }\end{array}$ \\
\hline $\begin{array}{l}\text { LIBERTY ASTHMA } \\
\text { VENTURE, 2020; Rabe } \\
\text { et al. (2020) }\end{array}$ & Phase 3 & $\begin{array}{l}\text { Severe uncontrolled } \\
\text { asthma }\end{array}$ & $\begin{array}{l}<300 \text { cells } / \mu \text { or } \\
\geq 300 \text { cells } / \mu \text { PB } \\
\text { eosinophils }\end{array}$ & $\begin{array}{l}\text { Biologic: Dupilumab } \\
\text { Dose: } 300 \mathrm{mg} \\
\text { Frequency: Q2W } \\
\text { Route: SC } \\
\text { Duration: } 24 \mathrm{~W}\end{array}$ & $\begin{array}{l}\text {-Improved pre-BD FEV } 1 \text {, } \\
\text { FVC, FEV } 1 / \text { FVC } \\
\text { - Reduced AAER }\end{array}$ & $-N / A$ \\
\hline Maspero et al. (2020) & Phase 3 & $\begin{array}{l}\text { Moderate-severe } \\
\text { uncontrolled asthma } \\
\text { and CRS }\end{array}$ & $\begin{array}{l}\geq 150 \text { cells } / \mu \text { or } \\
\geq 300 \text { cells } / \mu \mathrm{l} \text { PB } \\
\text { eosinophils }\end{array}$ & $\begin{array}{l}\text { Biologic: Dupilumab } \\
\text { Dose: 200, } 300 \text { mg } \\
\text { Frequency: Q2W } \\
\text { Route: SC } \\
\text { Duration: } 24 \mathrm{~W}\end{array}$ & $\begin{array}{l}\text {-Increased pre- and post- } \\
\text { BD FEV }_{1} \text { in CRS and non- } \\
\text { CRS groups } \\
\text {-Improved ACQ, AQLQ, } \\
\text { SNOTT-22 scores in CRS } \\
\text { and non-CSR groups }\end{array}$ & $\begin{array}{l}\text {-Decrease in FeNO, serum } \\
\text { IgE and TARC in CRS and } \\
\text { non-CRS groups } \\
\text {-No change in PB } \\
\text { eosinophils in non-CRS } \\
\text { group, but mild elevation in } \\
\text { CRS group }\end{array}$ \\
\hline
\end{tabular}

et al., 2003). Subsequent RCTs in severe asthmatics with blood eosinophils $\geq 400$ cells $/ \mu \mathrm{l}$, have shown that reslizumab reduces AAER by $50-60 \%$, improves symptom scores and lung function, and reduces blood eosinophils (Table 3) (Castro et al., 2011; Castro et al., 2015; Bjermer et al., 2016; Corren et al., 2016; Brusselle et al., 2017; Máspero, 2017; Murphy et al., 2017; Bernstein et al., 2020). Finally, post-hoc analysis suggested that patients who do not respond to the fixed-dose regimen of mepolizumab may benefit from reslizumab as an alternative (Mukherjee et al., 2018b). Similar to mepolizumab, reslizumab seems to have the most evidence for clinical efficacy in those with peripheral eosinophilia, however, the reduction in exacerbation rates remains incomplete.

Benralizumab, an antibody to the alpha subunit of the IL-5 receptor, was the third IL-5 pathway-targeting biologic that came to fruition. Phase $3 \mathrm{RCT}$ s have shown that severe eosinophilic asthmatics ( $\geq 150$ cells/ $\mu$ l blood eosinophils in last 12 months) treated with benralizumab $30 \mathrm{mg}$ SC q4 weeks, were able to reduce their prednisone dosing, exhibited reduced AAER by $28-55 \%$, and also showed improved lung function and symptom scores. From a cellular and molecular standpoint, benralizumab reduced peripheral and sputum eosinophils, along with diminished eosinophil products, including ECP and EDN (Nowak et al., 2015; FitzGerald et al., 2016; Sehmi et al., 2018). Expanding on this, benralizumab can also deplete basophils within peripheral blood in uncontrolled asthma (Eck et al., 2014), however a new study has shown that in mild asthmatics there is no depletion of peripheral or sputum basophils post-allergen provocation (Gauvreau et al., 2021). Although benralizumab uses an alternative approach of targeting the IL-5Ra, there still remains an incomplete ablation of exacerbation rates, similar to mepolizumab and reslizumab (Table 4).

\subsubsection{Possible Reasons for Suboptimal Responses With Anti-IL-5 Pathway Biologics}

This brings to question why there is only a partial reduction in exacerbation rates in severe asthmatics treated with anti-IL-5RAa biologics. There are several reasons for sub-optimal or failure of response which will be described below. First, it is important to understand that severe asthmatics eligible for biologics are already on ICS therapy. We know from multiple reports that nonadherence to ICS in severe asthmatics is substantial (Gamble et al., 2009; Lee et al., 2018; Sulaiman et al., 2018), and that nonadherent patients receiving mepolizumab have worse clinical outcomes (d'Ancona et al., 2020). This brings up to two interesting propositions. The first is that worsened underlying disease as a result of noncompliance with ICS is associated with uncontrolled inflammation, and this may make it more difficult for biologics targeting eosinophils to have a noticeable clinical impact in patients with suboptimal responses. Secondly, it is important to emphasize that corticosteroids target other aspects of T2-high inflammation that are not inhibited by anti-IL-5 biologics, including IL-4 and IL-13 activity (Ray et al., 2016). One can postulate that if additional T2-high inflammatory pathways beyond the IL-5 pathway are kept under control in 
TABLE 6 | Summary of randomized clinical trials assessing tezepelumab in severe asthma.

\begin{tabular}{|c|c|c|c|c|c|c|}
\hline \multicolumn{7}{|l|}{ Anti-TSLP: tezepelumab } \\
\hline $\begin{array}{l}\text { Landmark study } \\
\text { and year }\end{array}$ & $\begin{array}{c}\text { RCT } \\
\text { phase }\end{array}$ & Asthma severity & Biomarker & $\begin{array}{c}\text { Dosing, } \\
\text { duration } \\
\text { and route } \\
\text { of } \\
\text { administration }\end{array}$ & Clinical effect & Molecular effect \\
\hline Gauvreau et al. (2014) & $\begin{array}{l}\text { Phase } \\
1 b\end{array}$ & $\begin{array}{l}\text { Mild allergic } \\
\text { asthma }\end{array}$ & $\begin{array}{l}\text { Atopic, independent } \\
\text { of PB Eosinophils }\end{array}$ & $\begin{array}{l}\text { Dosing: } \\
700 \text { mg Q4W } \\
\text { Route: SQ } \\
\text { Duration: } 12 \mathrm{~W}\end{array}$ & $\begin{array}{l}34 \% \text { improvement in } \mathrm{FEV}_{1} \text { on } \\
\text { day } 84(p=0.02) \text { compared to } \\
\text { placebo }\end{array}$ & $\begin{array}{l}\text { - PB eosinophils declined post- } \\
\text { dosing and reached normal } \\
\text { levels by } 4 \mathrm{~W} \\
\text { - SP eosinophils reached } \\
\text { normal levels by } 6 \text { weeks } \\
\text { - FeNO levels improved } 1 \mathrm{~W} \\
\text { post-first dose } \\
\text { - No effect on total lgE levels }\end{array}$ \\
\hline $\begin{array}{l}\text { PATHWAY, 2017, 2021, 2021; } \\
\text { Corren et al. (2017); Corren et al. } \\
\text { (2021a); Corren et al. (2021b) }\end{array}$ & $\begin{array}{l}\text { Phase } \\
2 b\end{array}$ & $\begin{array}{l}\text { Severe } \\
\text { uncontrolled } \\
\text { asthma }\end{array}$ & $\begin{array}{l}\text { PB Eosinophils } \geq 250 \\
\text { or }<250 \text { cells } / \mu l\end{array}$ & $\begin{array}{l}\text { Dosing } \\
700 \text { mg Q4W } \\
210 \text { mg Q4W } \\
280 \text { mg Q2W } \\
\text { Route: SQ } \\
\text { Duration: } 52 \mathrm{~W}\end{array}$ & $\begin{array}{l}\text { - } 62-71 \% \text { reduction in } \\
\text { exacerbation irrespective of } \\
\text { phenotype, across all } \\
\text { seasons } \\
\text { - Reduced asthma- } \\
\text { exacerbation related } \\
\text { hospitalizations } \\
\text { - FEV } 120-150 \mathrm{ml} \\
\text { improvement vs. placebo ( } p= \\
\text { 0.002-0.015) } \\
\text {-Significant improvement in } \\
\text { ACQ and AQLQ scores in } \\
\text { higher-dose intervention arms }\end{array}$ & $\begin{array}{l}\text { - Decrease in PB eosinophils in } \\
\text { all tezepelumab groups at } 4 \mathrm{~W} \\
\text { onwards } \\
\text { - Decrease in total serum IgE all } \\
\text { tezepelumab groups }\end{array}$ \\
\hline $\begin{array}{l}\text { NAVIGATOR, 2021; } \\
\text { Menzies-Gow et al. (2021) }\end{array}$ & Phase 3 & $\begin{array}{l}\text { Severe } \\
\text { uncontrolled } \\
\text { asthma }\end{array}$ & $\begin{array}{l}<300 \text { cells } / \mu \text { or } \\
\geq 300 \text { cells } \mu \mathrm{l} \text { PB } \\
\text { eosinophils }\end{array}$ & $\begin{array}{l}\text { Dosing } \\
210 \text { mg Q4W } \\
\text { Route: SQ } \\
\text { Duration: } 52 \mathrm{~W}\end{array}$ & $\begin{array}{l}\text { - Reduced AAER by } 44-59 \% \\
\text { vs. placebo, irrespective of } \\
\text { phenotype } \\
\text { - FEV } 1230 \mathrm{ml} \text { improvement } \\
\text { vs. placebo ( } p<0.0001) \\
\text { - Significant improvement in } \\
\text { ACQ and AQLQ }\end{array}$ & $\begin{array}{l}\text { - Decrease in PB eosinophils } \\
\text { and FeNO levels at } 2 \mathrm{~W} \\
\text { onwards vs. placebo } \\
\text { - Serum lgE levels reduced over } \\
5 \mathrm{~W} \text { vs. placebo }\end{array}$ \\
\hline $\begin{array}{l}\text { UPSTREAM (2021); Sverrild et al. } \\
\text { (2021) }\end{array}$ & Phase 2 & $\begin{array}{l}\text { Severe } \\
\text { uncontrolled } \\
\text { asthma }\end{array}$ & $\begin{array}{l}\text { Independent of blood } \\
\text { eosinophils or atopic } \\
\text { status }\end{array}$ & $\begin{array}{l}\text { Dosing: } \\
700 \text { mg Q4W } \\
\text { Route: SQ } \\
\text { Duration: } 12 \mathrm{~W}\end{array}$ & $\begin{array}{l}\text { - Mean change in } \mathrm{PD}_{15} \\
\text { significantly reduced } \\
\text { - Non-significant } \\
\text { improvement in } \mathrm{ACQ}\end{array}$ & $\begin{array}{l}\text { - Airway tissue and BAL } \\
\text { eosinophils decreased by } 74 \\
\text { and } 75 \% \text {, respectively ( } p= \\
0.004, p=0.01 \text { ) } \\
\text { - No significant change in tissue } \\
\text { mast cells } \\
\text { - Subepithelial neutrophils } \\
\text { increased by } 51 \% \text { with } \\
\text { tezepelumab vs. } 33 \% \text { in } \\
\text { placebo (non-significant) }\end{array}$ \\
\hline $\begin{array}{l}\text { CASCADE, } 2020 \text { (Ongoing), } \\
2021 \text { Diver et al. (2021) }\end{array}$ & Phase 2 & $\begin{array}{l}\text { Moderate-severe } \\
\text { uncontrolled } \\
\text { asthma }\end{array}$ & $\begin{array}{l}<300 \text { cells } / \mu \text { or } \\
\geq 300 \text { cells } \mu \mathrm{l} \mathrm{PB} \\
\text { eosinophils }\end{array}$ & $\begin{array}{l}\text { Dosing: } \\
210 \text { mg Q4W } \\
\text { Route: SQ } \\
\text { Duration: } 28 \text { W }\end{array}$ & $\begin{array}{l}\text {-Reduced AHR to mannitol } \\
\text { vs. placebo }\end{array}$ & $\begin{array}{l}\text { - Decreased submucosal } \\
\text { eosinophils vs. placebo, } \\
\text { regardless of baseline PB } \\
\text { eosinophils } \\
\text { - No difference in } \mathrm{CD}^{+} \mathrm{T} \text { cells or } \\
\mathrm{CD}^{+} \mathrm{T} \text { cells, mast cells } \\
\text { - No difference in reticular } \\
\text { basement membrane thickness } \\
\text { and epithelial integrity }\end{array}$ \\
\hline $\begin{array}{l}\text { SOURCE, } 2020 \text { (Ongoing) } \\
\text { Wechsler et al. (2020b) }\end{array}$ & Phase 3 & $\begin{array}{l}\text { Severe } \\
\text { uncontrolled } \\
\text { asthma }\end{array}$ & $\begin{array}{l}<300 \text { cells } / \mu \text { or } \\
\geq 300 \text { cells } \mu \mathrm{PB} \\
\text { eosinophils }\end{array}$ & $\begin{array}{l}\text { Dosing: } \\
210 \text { mg Q4W } \\
\text { Route: SQ } \\
\text { Duration: } 48 \mathrm{~W}\end{array}$ & Pending & Pending \\
\hline $\begin{array}{l}\text { DESTINATION, } 2020 \text { (Ongoing) } \\
\text { Menzies-Gow et al. (2020) }\end{array}$ & Phase 3 & $\begin{array}{l}\text { Severe } \\
\text { Uncontrolled } \\
\text { Asthma }\end{array}$ & $\begin{array}{l}<300 \text { cells } / \mu \text { or } \\
\geq 300 \text { cells } \mu \mathrm{l} \text { PB } \\
\text { eosinophils }\end{array}$ & $\begin{array}{l}\text { Dosing: } \\
210 \text { mg Q4W } \\
\text { Route: SQ } \\
\text { Duration: } 36 \mathrm{~W}\end{array}$ & Pending & Pending \\
\hline
\end{tabular}


TABLE 7 | Summary of IL-33/ST2-targeted therapy in severe asthma.

\begin{tabular}{|c|c|c|c|c|c|c|}
\hline \multicolumn{7}{|l|}{ Anti-IL-33/ST2 } \\
\hline $\begin{array}{l}\text { Landmark study } \\
\text { and year }\end{array}$ & $\begin{array}{c}\text { RCT } \\
\text { phase }\end{array}$ & Disease model & Disease phenotype & $\begin{array}{l}\text { Dosing, duration } \\
\text { and route } \\
\text { of } \\
\text { administration }\end{array}$ & Clinical effect & Molecular effect \\
\hline Chen et al. (2019) & $\begin{array}{l}\text { Phase } \\
2 a\end{array}$ & Atopic dermatitis & Atopic & $\begin{array}{l}\text { Biologic: } \\
\text { Etokimab } \\
\text { Dosing: } 300 \mathrm{mg} \\
\text { Route: IV }\end{array}$ & $\begin{array}{l}-83 \% \text { achieved EASI50 } \\
\text { and 33\% EASI75 }\end{array}$ & $\begin{array}{l}\text { - Reduction in PB eosinophils at day } 29 \\
\text { - Reduction in skin neutrophil infiltration } \\
\text { post-HDM skin challenge } \\
\text { - Inhibited neutrophil migration to skin }\end{array}$ \\
\hline $\begin{array}{l}\text { Chinthrajah et al. } \\
\text { (2019) }\end{array}$ & $\begin{array}{l}\text { Phase } \\
2 a\end{array}$ & Peanut allergy & Atopic & $\begin{array}{l}\text { Biologic: } \\
\text { Etokimab } \\
\text { Dosing: } 300 \mathrm{mg} \\
\text { Route: IV } \\
\text { Duration: } 6 \mathrm{~W}\end{array}$ & $\begin{array}{l}\text { - Significant } \\
\text { desensitization to } \\
\text { peanuts }\end{array}$ & $\begin{array}{l}\text { - Reduction in cytokine levels (IL-4, IL-5, } \\
\text { IL-9, IL-13), and ST2 levels in CD4 }{ }^{+} T \text { cells } \\
\text { in PB } \\
\text { - Reduction in IgE at day } 15\end{array}$ \\
\hline $\begin{array}{l}\text { Wechsler et al. } \\
\text { (2021) }\end{array}$ & Phase & $\begin{array}{l}\text { Moderate-severe } \\
\text { asthma }\end{array}$ & $\begin{array}{l}<200 \text { cells } / \mu \text { l or } \\
\geq 200 \text { cells } \mu \mathrm{l} \text { PB } \\
\text { eosinophils }\end{array}$ & $\begin{array}{l}\text { Dosing: } 300 \mathrm{mg} \\
\text { Route: } \mathrm{SQ} \\
\text { Duration: } 12 \mathrm{~W}\end{array}$ & $\begin{array}{l}\text { - Reduction in loss of } \\
\text { asthma control (22\%) } \\
\text { - No improvement in } \\
\text { FEV1 } \\
\text { - Improvement in } \\
\text { AQLQ and ACQ }\end{array}$ & $\begin{array}{l}\text { - Reduction in mean blood eosinophil } \\
\text { count, FeNO, serum total lgE, periostin, } \\
\text { plasma eotaxin-3, and serum pulmonary } \\
\text { and activation-regulated chemokine } \\
\text { (PARC) }\end{array}$ \\
\hline $\begin{array}{l}\text { NCT03207243, } \\
2020 \text { (Ongoing) }\end{array}$ & $\begin{array}{l}\text { Phase } \\
2 \mathrm{a}\end{array}$ & $\begin{array}{l}\text { Moderate-severe } \\
\text { asthma }\end{array}$ & $\begin{array}{l}\text { Independent of blood } \\
\text { eosinophils or atopic } \\
\text { status }\end{array}$ & $\begin{array}{l}\text { Biologic: } \\
\text { GSK3772847 } \\
\text { Dosing: } \\
10 \mathrm{mg} / \mathrm{kg} \text { Q4W } \\
\text { Route: IV } \\
\text { Duration: } 28 \mathrm{~W}\end{array}$ & Pending & Pending \\
\hline $\begin{array}{l}\text { NCT02918019, } \\
2020 \text { (Ongoing) }\end{array}$ & $\begin{array}{l}\text { Phase } \\
2 b\end{array}$ & $\begin{array}{l}\text { Severe } \\
\text { uncontrolled } \\
\text { asthma }\end{array}$ & $\begin{array}{l}\text { Independent of blood } \\
\text { eosinophils or atopic } \\
\text { status }\end{array}$ & $\begin{array}{l}\text { Biologic: } \\
\text { MSTT1041A } \\
\text { Dosing: } 210 \mathrm{mg} \\
\text { Q4W } \\
\text { Route: SQ } \\
\text { Duration: } 50 \mathrm{~W}\end{array}$ & Pending & Pending \\
\hline
\end{tabular}

ICS-treated patients, then the beneficial effects of anti-IL-5 biologics may be amplified. Thus, continued and proper use of ICS is critical to dampening multiple T2-high endotypes that are not specifically targeted with anti-IL-5 biologics.

Now for a second proposal, there may be other inflammatory cells and cytokines, outside of the eosinophil-IL-5 pathway, that are equally, if not more important. For example, ILC2s, potent sources of IL-5, IL-9, IL-13, and PGD2, have been found to be higher in blood and sputum of severe asthmatics on high-dose steroids compared to mild asthmatics (Smith et al., 2016), and that these numbers are even higher in those with uncontrolled eosinophilia despite OCS. With respect to specifically targeting ILC2s, there have been a number of studies which have assessed the effect of corticosteroids and biologics on ILC2 numbers in severe asthma. Treatment with ICS has been shown to reduce ILC2-mediated inflammation, as well as ILC2 in nasal polyps, peripheral blood and sputum in asthma and asthma with allergic rhinitis (Walford et al., 2014; Yu et al., 2018). The corticosteroidresponsiveness of ILC2s may be dependent on activation by upstream cytokines including IL-33 and TSLP. This is supported by in vitro findings that IL-5 production from IL33-induced ILC2s can be attenuated by corticosteroids, but not when ILC2s are stimulated by TSLP (Liu et al., 2018). Not unexpectedly, although anti-IL-5 agents reduce total sputum IL-5 levels, they do not attenuate ILC2s within sputum or blood, suggesting that these biologics neutralize IL-5 production from these cells but do not affect their overall function within the airways (Sehmi et al., 2018; Mukherjee et al., 2018b). Given the importance of ILC2s in asthma, there is a need to develop treatments that specifically target the function of these cells, and as in vitro studies have shown, this may be done most effectively at an upstream level with targeting of alarmins. Another consideration is that there are alternative signalling pathways, independent of alarmins, that can activate ILC2s, including the TNF superfamily pathway, including the TL1A/ DR3 axis (Machida et al., 2020). Sputum TL1A levels are present in approximately $50 \%$ of prednisone-dependent severe asthmatics with uncontrolled eosinophilia (Machida et al., 2020). TL1A-induced activation of ILC2s, in the presence of TSLP and IL-2 is not responsive to dexamethasone (Machida 
TABLE 8 | Summary of other therapeutic targets in severe asthma.

\begin{tabular}{|c|c|c|c|c|c|c|}
\hline $\begin{array}{l}\text { Landmark study } \\
\text { and year }\end{array}$ & $\begin{array}{c}\text { RCT } \\
\text { phase }\end{array}$ & Disease severity & Disease phenotype & $\begin{array}{l}\text { Dosing, duration } \\
\text { and route } \\
\text { of administration }\end{array}$ & Clinical effect & Molecular effect \\
\hline $\begin{array}{l}\text { KRONOS, } 2020 \text { Levine } \\
\text { et al. (2020) } \\
\text { Anti-Siglec } 8\end{array}$ & $\begin{array}{l}\text { Phase } \\
1 \mathrm{~b}\end{array}$ & $\begin{array}{l}\text { Severe allergic } \\
\text { conjunctivitis }\end{array}$ & Atopic & $\begin{array}{l}\text { Biologic: AK002 } \\
\text { Dose: } 0.3,1, \\
3 \mathrm{mg} / \mathrm{kg} \text { q4W } \\
\text { Route: IV } \\
\text { Duration: } 6 \mathrm{M}\end{array}$ & $\begin{array}{l}\text { - ACQ score improved } \\
\text { by } 74 \% \text { vs. placebo } \\
\text { - } 72 \% \text { reduction in } \\
\text { asthma sx }\end{array}$ & $-\mathrm{N} / \mathrm{A}$ \\
\hline Hirano et al. (2020) & Phase 2 & $\begin{array}{l}\text { Eosinophilic gastritis } \\
\text { and esophagitis }\end{array}$ & Atopic & Biologic: AKOO2 & $\begin{array}{l}\text { - Improvement in } \\
\text { dysphagia symptom } \\
\text { scores }\end{array}$ & $\begin{array}{l}\text { - Reduction in esophageal } \\
\text { eosinophils }\end{array}$ \\
\hline Anti-Siglec 8 & & & & $\begin{array}{l}\text { Dose: } 0.3,1, \\
3 \mathrm{mg} / \mathrm{kg} \mathrm{q} 4 \mathrm{~W} \\
\text { Route: IV } \\
\text { Duration: } 4 \mathrm{M}\end{array}$ & & \\
\hline $\begin{array}{l}\text { EXHALE, } 2017 \text { Prussin } \\
\text { et al. (2017) } \\
\text { Dexpramipexole }\end{array}$ & Phase 2 & $\begin{array}{l}\text { Moderate-severe } \\
\text { asthma }\end{array}$ & $\begin{array}{l}\geq 300 \text { cells } \mu \mathrm{l} \text { PB } \\
\text { eosinophils }\end{array}$ & $\begin{array}{l}\text { Dose: } \\
\text { 75-300 mg/day } \\
\text { Route: PO } \\
\text { Duration: } 12 \mathrm{~W}\end{array}$ & $\begin{array}{l}\text { - Improved Pre-BD FEV } 1 \\
\text { from baseline }\end{array}$ & $\begin{array}{l}\text { - Reduced PB eosinophils } \\
\text { at } 12 \mathrm{~W}\end{array}$ \\
\hline $\begin{array}{l}\text { LUSTER 1\&2, } \\
2021 \text { Brightling et al. } \\
\text { (2021b) } \\
\text { Fevipiprant }\end{array}$ & Phase 3 & Severe Asthma & $\begin{array}{l}\geq 250 \text { or }<250 \text { cells } \mu \mathrm{l} \\
\text { PB eosinophils }\end{array}$ & $\begin{array}{l}\text { Dose: } \\
\text { 150-450 mg/day } \\
\text { Route: PO } \\
\text { Duration: } 52 \text { W }\end{array}$ & $\begin{array}{l}\text { - Improved AAER in } \\
\text { eosinophil high patients }\end{array}$ & $-N / A$ \\
\hline $\begin{array}{l}\text { Gonem et al. (2016) } \\
\text { Fevipiprant }\end{array}$ & Phase 2 & $\begin{array}{l}\text { Moderate-Severe } \\
\text { Asthma }\end{array}$ & $\geq 2 \%$ SP eosinophils & $\begin{array}{l}\text { Dose: } 250 \text { mg BID } \\
\text { Route: PO } \\
\text { Duration: } 12 \mathrm{~W}\end{array}$ & $\begin{array}{l}\text { - Favourable safety } \\
\text { profile }\end{array}$ & $\begin{array}{l}\text { - } 4.5 \text { times reduction in SP } \\
\text { eosinophils }\end{array}$ \\
\hline $\begin{array}{l}\text { Bateman et al. (2017) } \\
\text { Fevipiprant }\end{array}$ & Phase 2 & $\begin{array}{l}\text { Moderate-Severe } \\
\text { Asthma }\end{array}$ & $\operatorname{lgE} \geq 0.35 \mathrm{IU} \mathrm{mEq}$ & $\begin{array}{l}\text { Dose: } 1-450 \mathrm{mg} \\
\text { OD or BID } \\
\text { Route: PO } \\
\text { Duration: } 12 \mathrm{~W}\end{array}$ & $\begin{array}{l}\text { - Improved pre-BD FEV } 1 \\
\text { at } 12 \mathrm{~W}\end{array}$ & $-N / A$ \\
\hline Brightling et al. (2021a) & Phase 2 & Severe asthma & & Dose: 90 mg q4W & $\begin{array}{l}\text { - Shorter time to asthma } \\
\text { worsening }\end{array}$ & $\begin{array}{l}\text { - No change in blood } \\
\text { eosinophils, sputum } \\
\text { eosinophils or neutrophils }\end{array}$ \\
\hline
\end{tabular}

Duration: $24 \mathrm{~W}$

et al., 2020). This further substantiates the heterogeneity of asthmatics, and highlights the hypothesis that the cytokine milieu within the airway determines responsiveness to corticosteroids and biologics. Aside from ILC2s, there are other important immune pathways to consider. For example, basophils have been shown to produce marked levels of IL-4 and IL-13 within the airways (Salter et al., 2015; Salter et al., 2016). Basophils are not only activated through an IgE-dependent pathway but also by alarmin cytokines including TSLP, IL-33, and IL-25 (Salter et al., 2015; Salter et al., 2016). Interestingly, in severe asthma, there is increased expression of receptors for IL-33 and IL-25 on basophils, particularly after IgE stimulation (Boita et al., 2018). Thus, alarmin cytokines can not only activate basophils in an IgE-independent manner, but IgE itself can upregulate receptor expression for alarmin cytokines, creating a vicious cycle. Although basophils have been found to express the IL-5 receptor, there is mixed evidence as to whether anti-IL-5 agents can affect basophil function. For example, Wright et al.
(2019) found that 16 weeks of treatment with mepolizumab does not affect blood basophils in severe asthma. Similar to basophils, mast cells (MC) undergo extensive degranulation in fatal asthma suggesting that these cells are highly activated in severe asthma. Through both IgE-dependent and -independent mechanisms, MC not only release mediators such as histamine, prostaglandin and leukotrienes, but also produce a wide range of cytokines, including IL-4, IL-13, IL-6, IL-17, and TSLP (Bradding et al., 1992; Bradding et al., 1994; Ying et al., 1995; Ying et al., 2005). Collectively, there are numerous immune cells, outside of eosinophils, which can contribute to inflammatory processes inherent in severe asthma, independent of IL-5. Lastly, in addition to cytokines, there are granule proteins produced by eosinophils that play an important role in promoting airway obstruction. For example, EPX, a product of eosinophils, utilizes respiratory burst-derived $\mathrm{H}_{2} \mathrm{O}_{2}$ to generate reactive oxidants that can kill pathogens or activate airway cells (Wu et al., 2000). EPX has been shown to promote mucus plug formation by generating 


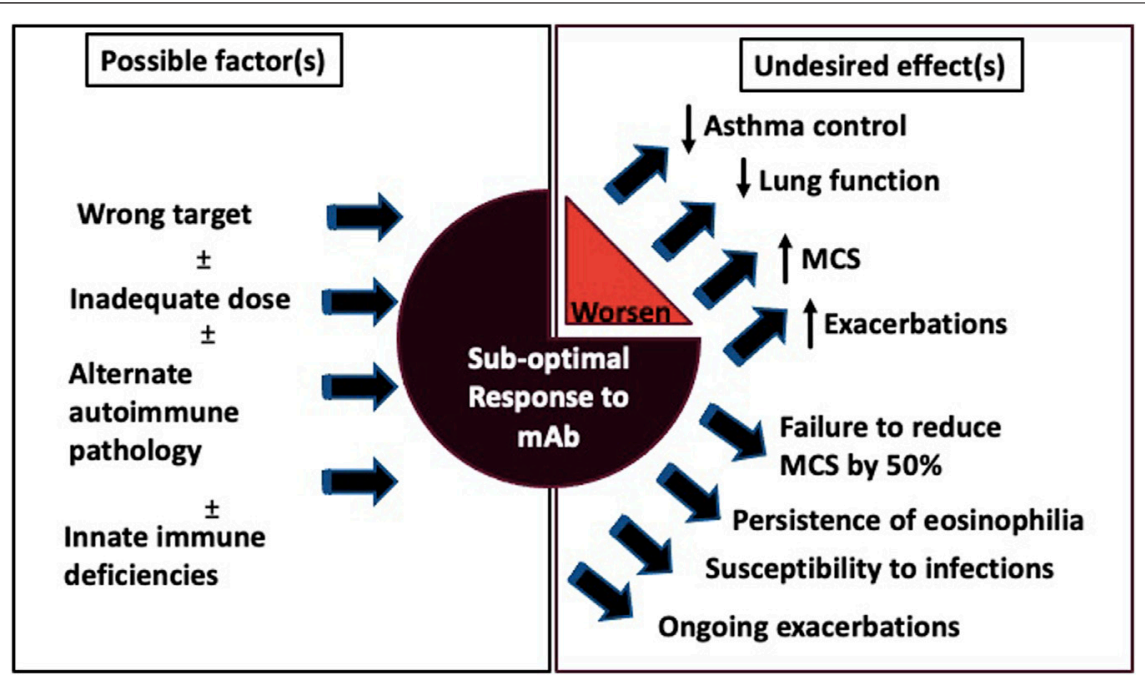

FIGURE 2 | Factors affecting optimal biologic response and clinical ramifications. The schematic addresses the primary factors that alone or in combination can lead to sub-optimal treatment response to currently approved monoclonal antibodies (mAbs) in severe asthma. The sub-optimal responses to mAbs can result in undesired clinical manifestations via, persistence and worsening of asthma symptoms, exacerbations, infections and decline in lung functions. Abbreviations: mAb: Monoclonal anitbody; MCS: Maintenance corticosteroids.

oxidants that modify mucins. Mucus plugging has been found in severe asthmatics and may predispose to infection (Dunican et al., 2018). Biologic therapy may not effectively target mucus plugging and is important to identify in patients with recurrent infections.

Other reasons for underlying suboptimal responses to biologics are related to dosing regimen and routes of administration. Mepolizumab has fixed dosing with SC administration versus reslizumab with IV weight-based dosing. Notably, the clinical benefits are the same for high and low doses of mepolizumab that is administered either IV or SC in severe asthmatics requiring high dose ICS (Mukherjee et al., 2018b). Conversely, severe asthmatics requiring daily prednisone have better clinical outcomes with higher doses of IV over lower doses of SC administration (Mukherjee et al., 2018b). These findings may be explained by lower doses administered SC not adequately neutralizing IL-5 within the airways, despite attenuated peripheral eosinophilia. For example, patients treated with 4 doses of weightadjusted IV reslizumab after previously being treated with SC mepolizumab, resulted in suppressing both airway and peripheral eosinophilia (Mukherjee et al., 2018b). The magnitude of attenuation with reslizumab was greater compared to treatment with 12 doses of mepolizumab. The suppression of eosinophils and progenitors coincided with clinical improvement, as shown by increased $\mathrm{FEV}_{1}$ and asthma control. Patients who did not respond to anti-IL-5 treatment had higher sputum IL-5 levels. Collectively, this suggests that a greater concentration of anti-IL- 5 either through increased dose or IV administration, is needed to neutralize T2-high inflammation, that is driven not only by eosinophils but also ILC2s, in peripheral and local airway compartments. Another hypothesis to consider is that low doses of anti-IL-5 biologics may cause worsening of airway eosinophilia through inducing immune-complex (IC) formation or complement consumption (Mukherjee and Nair, 2018; Mukherjee et al., 2020). ICs may act like cytokine depots, leading to increased potency of bound IL-5, resulting in worsening symptoms. We have seen detectable levels of sputum immunoglobulin-bound IL-5 in mepolizumab non-responders, coinciding with increased sputum IL-5. We only observed this immunoglobulin-IL-5 phenomenon in one patient receiving reslizumab, suggesting that this issue is prevented with higher dosing and/or IV delivery.

This leads us to the next hypothesis that inadequate dosing of IgG1 neutralising antibodies have the potential for disease worsening, particularly in those with an underlying airway autoimmune component. There is emerging evidence that local airway autoimmune responses contribute to corticosteroid insensitivity in severe asthma (Mukherjee et al., 2017; Mukherjee and Nair, 2018). A third of eosinophilic asthmatics have airway autoimmune responses, manifesting as auto-IgG antibodies directed against cell-derived granule proteins including, EPX (Liu et al., 2018). Severe asthmatics with sputum autoantibodies have characteristic clusters of sputum FEGs, indicative of active eosinophil cytolysis (Liu et al., 2018). Interestingly, we have shown that patients treated with reslizumab had a reduction in sputum anti-EPX IgG and antinuclear antibody (ANA), but not with mepolizumab. In fact, an increase in sputum anti-EPX IgG was seen in $66 \%$ of non-responders to mepolizumab. An additional study supports these findings, where $60 \%$ of severe asthmatics treated with mepolizumab showed a suboptimal response versus 32\% with reslizumab (Mukherjee et al., 2020). In those who showed a poor response to mepolizumab, 23\% worsened clinically, and had higher levels of sputum Anti-EPX IgG levels. An extension of this study showed that $43 \%$ of patients on either mepolizumab or reslizumab had suboptimal responses, with $14 \%$ of these patients having worsening asthma (Mukherjee et al., 2020). We found increased sputum IL-5, anti-EPX, EPX, and C3c in those with suboptimal response to biologics. In addition, increased $\mathrm{C} 1-\mathrm{q} / \mathrm{IgG}$ levels and $\mathrm{C} 1-\mathrm{q}-$ IgG/IL-5-IgG dual-positive cells in sputum plugs were found in those who worsened on mepolizumab. This is supported by a case report 
where we described a severe asthmatic treated with mepolizumab had worsening of symptoms, and molecular analysis revealed increased anti-EPX and IL- $5^{+}$ILC2s, suggesting that increased Th2 signaling leads to activation of IL-5-producing ILC2s and subsequent eosinophilia (Mukherjee et al., 2017). This brings up an interesting theory that inadequate drug dosing results in hetero immune-complex formation of complement-fixing antibody that is bound to the $\mathrm{Clq}$ molecule (Duncan and Winter, 1988), which can induce the complement cascade and promote recruitment of immune cells via the Fc $\gamma \mathrm{R}$ receptor (Stokol et al., 2004). As such, there may be an autoimmune-triggered IC-mediated phenomena in those with worsened response to mepolizumab. Finally, the monitoring of blood eosinophils did not help to identify this subgroup, nor did assessment of autoimmunity biomarkers. Peripheral eosinophilia was only observed in $8 \%$ of suboptimal responders, whereas $6 \%$ continued to have increased sputum eosinophils $>3 \%$, representing nonattenuated airway eosinophilia, and $69 \%$ of these patients had sputum eosinophils despite normal blood eosinophils. These findings support a discordance between blood and sputum eosinophils, and while peripheral eosinophils may be adequate for selecting patients that may benefit from anti-IL- 5 therapy, it may be insufficient for monitoring therapeutic response to anti-IL-5 biologics (Mukherjee and Nair, 2015; Mukherjee et al., 2017; Mukherjee et al., 2018b; Drick et al., 2018; Ojanguren et al., 2018). Instead, we propose that sampling from the airways may be a more adequate way to identify monitor treatment response. For example, we have shown that sputum eosinophil count prior to treatment did not predict response to mepolizumab, nor was this the case with peripheral eosinophils. However, sputum eosinophils were effective for assessing response to treatment as early as 4 months posttreatment (Mukherjee et al., 2020). Of note, there is the emerging concept of "breathomics," which is the phenotyping of patients through non-invasive identification of exhaled volatile organic compounds (VOCs) using gas chromatography and mass spectrometry (De Vries et al., 2018; Sterk, 2019). Measurement of VOCs has been shown to have similar accuracy to sputum cell counts and FeNO (Sterk, 2019). This may be an alternative or additive approach to monitoring treatment response to biologic agents.

There is a subset of severe asthmatics with frequent respiratory infections that is thought to be secondary to underlying airway neutrophilia. There are higher levels of IgM and IgG in asthmatics compared to healthy controls with recurrent respiratory tract infections (Ho et al., 2020). Specifically, eosinophilic asthmatics have lower levels of IgA compared to healthy controls. We have shown that administration of IVIg leads to increased total IgG and subtypes, and these patients had fewer infective exacerbations over 12 months (Ho et al., 2020). These findings suggest that although eosinophilic inflammation may be dampened by biologics, exacerbations continue to occur due to underlying neutrophilia and humoral deficiency. It is important to consider assessing immunoglobulin levels in those with frequent asthmatics and replace if necessary. We have previously assessed severe asthmatic responses over 14 months to benralizumab, and found that $27 \%$ of patients had suboptimal responses and $40 \%$ of these patients had worsening disease (Poznanski et al., 2021). Only two patients with worsening asthma had sputum eosinophilia, whereas 16 had evidence of infective exacerbation with neutrophilic inflammation. A suboptimal response to benralizumab has been proposed to be due to impaired NK function and/or number (Poznanski et al., 2021). Overall, respiratory infections increased with benralizumab and had associated sputum neutrophilia, which is in contrast to mepolizumab or reslizumab that is associated with eosinophilic exacerbations. Previous history of infections predicted poor responses to benralizumab (Poznanski et al., 2021). Lastly, benralizumab is seemingly more potent than the other anti-IL-5 agents at suppressing airway eosinophilia. Although there have been no head-to-head trials with anti-IL-5 agents, benralizumab appears to be non-superior relative to mepolizumab or reslizumab from a clinical standpoint. As mentioned earlier, there are numerous other pathways, aside from the IL-5-eosinophil pathway which may contribute to asthma pathogenesis but may not be adequately attenuated by IL-5targeted biologics. For example, alternative Th2 cytokines, such as IL-4 and IL-13 or alarmin cytokines, and other immune cells such as basophils, Th2 cells, MC, and ILC2s may still be present and activated within the airways despite treatment with benralizumab. Thus, regardless of complete attenuation of eosinophils, there are other, redundant pathways that can carry out airway inflammation. The combination of anti-IL-5 agents with biologics that target other important Th2 pathways may confer better clinical outcomes, however this needs to be studied in more detail in the future.

\subsection{IL-4/IL-13 Targeted Therapy}

In order to understand the efficacy of IL-4/IL-13 agents, it is important to understand receptor signaling involved with these two cytokines. IL-13 signals through the IL-13 receptor, of which there are two subtypes, including IL-13Ra1 and IL-13Ra2. IL$13 \mathrm{Ra} 1$ binds to IL-13 with low affinity, but when the IL-4 receptor, IL-4Ra1, joins to form a heterodimer, IL-13 is bound with greater affinity. The IL-13Ra2, binds to IL-13 with high affinity but lacks a cytoplasmic domain thus does not signal downstream, however it may act as a negative regulator of IL-13 and IL- 4 signaling. While isolated blockade of either IL-4 or IL-13 has not been shown to be effective in treatment of severe asthma, dual blockade of IL-4 and IL-13 has shown promise.

With respect to anti-IL-13 biologics, two agents have been studied, lebrikizumab and tralokinumab. Studies looking at moderate-severe asthma with T2-high inflammation (total IgE $\geq 100 \mathrm{IU} / \mathrm{ml}$ and blood eosinophils $\geq 140 \mathrm{cells} / \mu \mathrm{l}$ ) have shown that treatment with lebrikizumab resulted in $60 \%$ reduction of AAER and improved $\mathrm{FEV}_{1}$, but no effect on symptoms (Corren et al., 2011). Subgroup analysis showed that patients with higher serum periostin $(\geq 50 \mathrm{ng} / \mathrm{ml})$ or FeNO had greater improvement in lung function. Furthermore, there was an observed decrease in FeNO and serum IgE, but not eosinophils. Larger RCTs have shown that severe asthmatics with T2-high biomarkers (serum periostin $\geq 50 \mathrm{ng} / \mathrm{ml}$ and/or blood eosinophils $\geq 300 \mathrm{cells} / \mu \mathrm{l}$ ) treated with lebrikizumab had reduced AAER but no coinciding improvement in symptom scores and only marginal improvement in $\mathrm{FEV}_{1}$ (Hanania et al., 2015; Hanania et al., 2016). Similarly, the majority of studies with tralokinumab did not show promising clinical outcomes (Piper et al., 2013; Brightling et al., 2015; Panettieri et al., 2018; Russell et al., 2018). Collectively, anti-IL-13-specific agents are not effective in treating severe asthma. This may be due to IL-13 primarily being involved with AHR as opposed to exacerbation and/or inflammation. 
With respect to tralokinumab, it targets both the IL-13Ra1 and IL$13 \mathrm{Ra} 2$ subunits, and thus may dampen the anti-inflammatory effect through IL-13Ra2 (Table 5).

The trials with respect to anti-IL-4 biologics have also been disappointing. Pascolizumab was shown to be well tolerated in animal studies with monkeys and effective in neutralizing bioactivity of IL-4 (Hart et al., 2002). Human RCTs have shown that treatment with Altrakincept, a nebulized anti-IL-4 agent, in moderate-severe asthmatics significantly improved in $\mathrm{FEV}_{1}$ and reduced FeNO, with no effect on AAER (Borish et al., 1999; Borish et al., 2001). Overall, anti-IL-4 agents have not yielded sufficient clinical efficacy to warrant further investigation and research has been halted. The lack of efficacy, may in part, be due to redundancy provided by IL-13, which signals through the same heterodimer.

Given the lack of impressive data from anti-IL-4 and IL-13 individual biologic agents, it was thought that perhaps targeting a common pathway between both cytokines may yield greater effect. Dupilumab is the first dual IL-4/IL-13 biologic approved for asthma treatment. It targets the shared IL-4Ra receptor and thus blocks signalling of both IL-4 and IL-13. An initial trial with dupilumab treatment in eosinophilic asthmatics (blood eosinophils $\geq 300$ cells $/ \mu$ l or sputum eosinophils $\geq 3 \%$ ) resulted in an $87 \%$ reduction in AAER (Wenzel et al., 2013). Of note, the treatment groups were instructed to stop their maintenance LABA at week 4 and wean from ICS from weeks 6-9. Exacerbations were only seen after the point of inhaler withdrawal, suggesting that dupilumab may be acting on the same pathway as ICS/LABA inhalers and hence has a redundant effect. A larger RCT with severe asthmatics (blood eosinophils $\geq 300 \mathrm{cells} / \mu \mathrm{l}$ ) over a 24 -week period showed $81 \%$ reduction in AAER compared to $60 \%$ reduction with the low eosinophilia group, both of which were significant compared to placebo. These findings suggest that dupilumab may be effective regardless of eosinophilic status. However, other RCTs showed that dupilumab treatment only yielded significant reduction in AAER in patients with blood eosinophils $\geq 150 \mathrm{cells} / \mu \mathrm{l}$ and FeNO $\geq 25 \mathrm{ppb}$. In terms of biomarkers to monitor response, dupilumab induces transient increases in blood eosinophils but significant reduction in FeNO, suggesting that FeNO may be a better biomarker to assess treatment eligibility and efficacy for this treatment.

\subsubsection{Possible Reasons for Suboptimal Responses with Anti-IL-4/IL-13}

There have been some case reports of adverse events when switching from anti-IL-5 to anti-IL-4/IL-13. For example, patients switched from anti-IL-5 to anti-IL-4/IL-13 had worsening in asthma control and showed increased use of OCS, with substantial increases in peripheral eosinophils (Eger et al., 2021). The reason for this worsening is not entirely understood, but the working hypothesis is that these patients had underlying anti-neutrophil cytoplasmic antibody (ANCA) negative EGPA triggered by the rebound hypereosinophilia brought on by dupilumab. We propose that both pathways (IL-5, IL-4/IL-13) will need to be targeted to allow for optimal disease control. Unfortunately, no studies thus far have looked at the efficacy of combining anti-IL-4/IL-13 and anti-IL-5 biologics for asthma treatment.
Sub-optimal responses to anti-IL-4/IL-13 may be explained by these agents primarily focusing on reducing AHR as opposed to dampening airway inflammation (Gour and Wills-Karp, 2015). We propose that dupilumab should be used in patients who have symptoms of AHR, and if there is overlap with airway inflammation, it may be reasonable to pair with an upstream inhibitor, such as an anti-IL-33 or anti-TSLP agent. In addition, it is well known that mucus hyperplasia is promoted by the IL-4/IL-13 axis (Munitz et al., 2008; Bao and Reinhardt, 2015) and as such, patients with mucus hypersecretion as a primary symptom should be managed with agents targeting this axis such as dupilumab.

Interestingly, Wechsler et al. assessed the treatment of severe asthmatics with a combination of dupilumab and anti-IL-33 (Wechsler et al., 2020a). The anti-IL-33 biologic on its own was able to improve asthma control and lung function, but this was not synergistic when combined with dupilumab. This may have been due to both agents having redundant T2-high pathways, whereas there was insufficient targeting of IL-5 or TSLP, and hence continued activity of ILC2s and Th2 cells. Alternatively, this study was not adequately powered for between group comparisons. This study certainly provides food for thought and warrants further investigation with respect to studying combined biologic regimens.

\subsection{Alarmin Cytokine Therapy}

The development of anti-alarmin biologics has been one of the most exciting innovations in asthma therapy to date. Tezepelumab is a human $\operatorname{IgG}_{2}$ antibody directed against TSLP, that can be administered IV or SC. The first landmark trial to assess the efficacy of tezepelumab was carried out in mild asthmatics, which showed significant improvement in $\mathrm{FEV}_{1}$ and reduction of peripheral and sputum eosinophils, along with decreased FeNO levels during the late phase response post-allergen provocation (Gauvreau et al., 2014). Subsequent RCTs in severe asthmatics showed that tezepelumab treatment resulted in a $44-71 \%$ reduction in AAER, irrespective of baseline peripheral eosinophilia (Table 6) (Marone et al., 2019; Corren et al., 2021a; Menzies-Gow et al., 2021; Sverrild et al., 2021). Patients were also shown to have improvements in $\mathrm{FEV}_{1}$ and symptoms, with a coinciding reduction in peripheral eosinophils and serum IgE. Collectively, these data suggest that targeting upstream cytokines, such as TSLP, may prove to be beneficial in multiple asthma endotypes, both within and outside of T2-high inflammation.

Initial studies that looked at anti-IL-33 agents were done in other sites of allergy beside asthma, specifically in atopic dermatitis and peanut allergy. Etokimab has been shown to improve symptoms related to atopic dermatitis and reduce desensitization to peanuts (Chen et al., 2019; Chinthrajah et al., 2019). These clinical findings were associated with significant reductions in peripheral eosinophils and T2 cytokine levels, along with total serum IgE. There are multiple RCTs underway assessing the efficacy of anti-IL-33 biologics to treat asthma, but most results are pending (Table 7). Of note, the first RCT was recently published in 2021 by Wechsler et al. which assessed the effect of an anti-IL-33 agent called itepekimab in a phase 2 trial with moderate-to-severe asthma (Wechsler et al., 2021). They found that following 12 weeks of treatment with itepekimab there was an improvement in asthma control and quality of life. Furthermore, 
loss of asthma control occurred in $22 \%$ of patients in the itepekimab group compared to $19 \%$ in the dupilumab group and $27 \%$ in the combined group. Interestingly, itepekimab alone, or in combination with dupilumab resulted in decreased blood eosinophil. Itepekimab alone was also able to reduce FeNO, serum total IgE, periostin, and plasma eotaxin- 4 but to a lesser extent compared to dupilumab or combined therapy. Overall, this anti-IL-33 agent reduced blood eosinophils to a lesser extent than anti-IL-5 agents and it had reduced effect on eotaxin-3, which is an IL-13 product, compared to dupilumab. These findings suggest incomplete inhibition of Th2 inflammation with blockade of IL-33, given that other alarmin cytokines are still active, including TSLP. More studies are needed to determine the effect of anti-IL-33 agents on airway eosinophils and other inflammatory parameters.

Overall, there have been not been sufficient RCTs with antialarmin agents to be able to identify suboptimal or failure to respond and what might be responsible for these outcomes. It will be helpful in the future to assess dual targeting of upstream and downstream inflammatory cytokines to treat severe asthma.

\subsection{Other Current Therapy}

There have been other agents that target various aspects of eosinophil function which have been assessed, albeit less extensively than the aforementioned biologics (Table 8). Sialicacid-binding immunoglobulin-like lectin (Siglec) -8 is a cell surface receptor found on mast cells and eosinophils. Initial studies have shown that an anti-Siglec-8 induces death of cytokine-primed eosinophils and inhibits IgE-mediated mast cell activation (Levine et al., 2020). RCTs with AK002 in severe allergic conjunctivitis reported improved symptoms (Levine et al., 2020), and these patients were also found to have comorbid asthma, with $72 \%$ reduction in asthma symptom scores. Hirano et al. assessed AK002 in eosinophilic gastritis and esophagitis, showing significant improvement in dysphagia symptoms and reduction in esophagus eosinophils (Table 8) (Hirano et al., 2020). Further studies are needed to determine the efficacy of anti-Siglec- 8 in asthma.

Dexpramipexole is a small molecule traditionally developed as treatment for amyotrophic lateral sclerosis. These studies showed significant and persistent reduction in blood eosinophils 1-2 months after drug initiation. The spotlight has now turned to assessing the use of dexpramipexole for asthma and CRS. Initial studies with CRS have shown that dexpramipexole can reduce peripheral eosinophils to $<0.020 \times 10^{9} / \mathrm{L}$ at month 6 postinitiation, and yield a $97 \%$ reduction in nasal polyp tissue eosinophils from baseline (Laidlaw et al., 2019). Similarly, another study showed that dexpramipexole can reduce blood eosinophils by $93 \%$ compared to baseline at month 6 and $94 \%$ in tissue nasal polyps (Prussin et al., 2017). In terms of asthma, 12 weeks treatment with dexpramipexole resulted in significant reduction of peripheral eosinophils and improved $\mathrm{FEV}_{1}$ (Prussin et al., 2021). More data is needed to determine effect on sputum eosinophils and whether this reduction translates into meaningful clinical outcomes.

Prostaglandin D2 receptor 2 (PGD2) is a potent mediator involved in asthma pathogenesis with the main function to promote airway smooth muscle contraction. In addition, PDG2 activates the DP2 chemokine receptor, also known as
CRTH2, on Th2 cells, ILC2s, granulocytes, and monocytes. The PGD2/CRTH2 axis is implicated in cell adhesion, survival, and activation resulting in cytokine/chemokine production, and subsequent downstream eosinophilia. Fevipiprant is an oral PGD2 receptor antagonist recently developed for asthma. Studies have shown that in moderate-severe asthma fevipiprant reduces sputum and bronchial submucosal eosinophils, and reduces airway smooth muscle mass compared with placebo (Saunders et al., 2019). Similar studies have shown that fevipiprant can induce a 3-5 greater 3-5 fold reduction in sputum eosinophils (Gonem et al., 2016; Saunders et al., 2019). From a clinical perspective, treatment with fevipiprant can improve pre-dose trough $\mathrm{FEV}_{1}$ and symptom scores, as well as reduce AAER (Erpenbeck et al., 2016). Conversely, other trials did not show improvement in symptom scores or $\mathrm{FEV}_{1}$ (Castro et al., 2021). Overall, findings have been inconsistent with respect to clinical efficacy of fevipiprant treatment. A recent systematic review confirms this by showing that although the agent is safe, it does not reach minimal clinically important difference (Yang et al., 2021). Suboptimal responses may be explained by the PGD2/CRTH2 axis being one of the many pathways that control Th2 and ILC2 activation, and that anti-alarmin pathways may be more crucial to target. In summary, there is a need for more studies determining the efficacy of combining these agents with other biologics.

Lastly, IL-23 has been implicated in airway inflammation that is mediated by Th2 and Th17 cytokines. Animal models have shown IL-23 to promote Th17 cell proliferation, which in turn maintains IL-17A and IL-17F production and neutrophil recruitment (Li and Hua, 2014). IL-23 also promotes Th2 cytokine production and eosinophil infiltration (Wakashin et al., 2008). Brightling et al. (2021a) recently conducted a phase 2a RCT assessing the effect of an anti-IL-23p19 agent, called risankizumab in severe asthma. They found that the time-to-first asthma worsening and rate ratio for annualized asthma worsening was shorter in the risankizumab group compared to placebo. There was no effect of risankizumab on FeNO, median blood eosinophil count or sputum eosinophils and neutrophils. They did, however, report that risankizumab reduced the sputum IL-23 gene set and pathways associated with activation of cytotoxic $\mathrm{T}$ cells and NK cells. Their findings challenge the proposed role of Th17 and IL-23 in severe asthma. The worsening in asthma control may have been attributed to targeting of IL-23 leading to an increase in Th2 mediators, such as IL13 , thereby resulting in increased smooth-muscle tone within the airways. Interestingly, the poorer outcomes in the risankizumab group were amplified in those with higher blood eosinophils counts. Given the evidence of potential harm related to IL-23 blockade, it may be reasonable to steer away from this area of study or consider studies assessing the efficacy of combining anti-IL-23 or anti-IL-17 agents with Th2-targeted agents.

\section{CONCLUSION}

Overall, we have described a number of mechanisms that could be contributing to failed or sub-optimal response to biologic 
therapy in severe asthma. The main themes are the need for proper dosing and route of administration of biologics, the identification of underlying inflammation through proper immune endotyping, as well as the targeting of multiple pathways, both upstream and downstream. An astute understanding of the molecular mechanisms and their associated clinical manifestations require careful consideration for development of valid biomarkers that will help guide optimal treatment and monitor therapeutic response. Moving forward, we need these biomarkers to assess individual patient symptoms and determine the underlying immunological mechanism that may be primarily responsible for driving disease severity and aid in choosing the right targeted therapy.

\section{REFERENCES}

Adachi, T., Choudhury, B. K., Stafford, S., Sur, S., and Alam, R. (2000). The Differential Role of Extracellular Signal-Regulated Kinases and P38 MitogenActivated Protein Kinase in Eosinophil Functions. J. Immunol. 165, 2198-2204. doi:10.4049/jimmunol.165.4.2198

Bagnasco, D., Milanese, M., Rolla, G., Lombardi, C., Bucca, C., Heffler, E., et al. (2018). The North-Western Italian Experience with Anti IL-5 Therapy Amd Comparison with Regulatory Trials. World Allergy Organ. J. 11, 34. doi:10.1186/ s40413-018-0210-7

Bao, K., and Reinhardt, R. L. (2015). The Differential Expression of IL-4 and IL-13 and its Impact on Type-2 Immunity. Cytokine 75, 25-37. doi:10.1016/ j.cyto.2015.05.008

Barnes, N., Menzies-Gow, A., Mansur, A. H., Spencer, D., Percival, F., Radwan, A., et al. (2013). Effectiveness of Omalizumab in Severe Allergic Asthma: A Retrospective UK Real-World Study. J. Asthma 50, 529-536. doi:10.3109/ 02770903.2013.790419

Barnett, S. B., and Nurmagambetov, T. A. (2011). Costs of Asthma in the United States: 2002-2007. J. Allergy Clin. Immunol. 127, 145-152. doi:10.1016/j.jaci.2010.10.020

Bateman, E. D., Guerreros, A. G., Brockhaus, F., Holzhauer, B., Pethe, A., Kay, R. A., et al. (2017). Fevipiprant, an Oral Prostaglandin DP2 Receptor (CRTh2) Antagonist, in Allergic Asthma Uncontrolled on Low-Dose Inhaled Corticosteroids. Eur. Respir. J. 50, 50. doi:10.1183/13993003.00670-2017

Bel, E. H., Wenzel, S. E., Thompson, P. J., Prazma, C. M., Keene, O. N., Yancey, S. W., et al. (2014). Oral Glucocorticoid-Sparing Effect of Mepolizumab in Eosinophilic Asthma. N. Engl. J. Med. 371, 1189-1197. doi:10.1056/ NEJMoa1403291

Bernstein, J. A., Virchow, J. C., Murphy, K., Maspero, J. F., Jacobs, J., Adir, Y., et al. (2020). Effect of Fixed-Dose Subcutaneous Reslizumab on Asthma Exacerbations in Patients with Severe Uncontrolled Asthma and Corticosteroid Sparing in Patients with Oral Corticosteroid-dependent Asthma: Results from Two Phase 3, Randomised, Double-Blind, PlaceboControlled Trials. Lancet Respir. Med. 8, 461-474. doi:10.1016/S22132600(19)30372-8

Bjermer, L., Lemiere, C., Maspero, J., Weiss, S., Zangrilli, J., and Germinaro, M. (2016). Reslizumab for Inadequately Controlled Asthma with Elevated Blood Eosinophil Levels: A Randomized Phase 3 Study. Chest 150, 789-798. doi:10.1016/j.chest.2016.03.032

Bleecker, E. R., FitzGerald, J. M., Chanez, P., Papi, A., Weinstein, S. F., Barker, P., et al. (2016). Efficacy and Safety of Benralizumab for Patients with Severe Asthma Uncontrolled with High-Dosage Inhaled Corticosteroids and LongActing $\beta 2$-agonists (SIROCCO): a Randomised, Multicentre, PlaceboControlled Phase 3 Trial. Lancet 388, 2115-2127. doi:10.1016/S01406736(16)31324-1

Boita, M., Heffler, E., Omedè, P., Bellocchia, M., Bussolino, C., Solidoro, P., et al. (2018). Basophil Membrane Expression of Epithelial Cytokine Receptors in

\section{AUTHOR CONTRIBUTIONS}

BS and MM wrote the manuscript draft. PL contributed to the final development of the manuscript. MM and PL conceived the review structure and take responsibility of the overall manuscript and information.

\section{FUNDING}

PL reports grants from AstraZeneca, Natural Sciences and Engineering Research Council (NSERC), Synergy Respiratory Care, and receives honoraria from AstraZeneca and GlaxoSmithKline outside this work. Grant funding number: EAA-167626.

Patients with Severe Asthma. Int. Arch. Allergy Immunol. 175, 171-176. doi: $10.1159 / 000486314$

Borish, L. C., Nelson, H. S., Corren, J., Bensch, G., Busse, W. W., Whitmore, J. B., et al. (2001). Efficacy of Soluble IL-4 Receptor for the Treatment of Adults with Asthma. J. Allergy Clin. Immunol. 107, 963-970. doi:10.1067/mai.2001.115624

Borish, L. C., Nelson, H. S., Lanz, M. J., Claussen, L., Whitmore, J. B., Agosti, J. M., et al. (1999). Interleukin-4 Receptor in Moderate Atopic Asthma. A Phase I/II Randomized, Placebo-Controlled Trial. Am. J. Respir. Crit. Care Med. 160, 1816-1823. doi:10.1164/ajrccm.160.6.9808146

Bradding, P., Feather, I. H., Howarth, P. H., Mueller, R., Roberts, J. A., Britten, K., et al. (1992). Interleukin 4 Is Localized to and Released by Human Mast Cells. J. Exp. Med. 176, 1381-1386. doi:10.1084/jem.176.5.1381

Bradding, P., Roberts, J. A., Britten, K. M., Montefort, S., Djukanovic, R., Mueller, R., et al. (1994). Interleukin-4, -5, and -6 and Tumor Necrosis Factor-Alpha in normal and Asthmatic Airways: Evidence for the Human Mast Cell as a Source of These Cytokines. Am. J. Respir. Cel Mol Biol 10, 471-480. doi:10.1165/ ajrcmb.10.5.8179909

Brightling, C. E., Chanez, P., Leigh, R., O’Byrne, P. M., Korn, S., She, D., et al. (2015). Efficacy and Safety of Tralokinumab in Patients with Severe Uncontrolled Asthma: a Randomised, Double-Blind, Placebo-Controlled, Phase 2b Trial. Lancet Respir. Med. 3, 692-701. doi:10.1016/S2213-2600(15) 00197-6

Brightling, C. E., Nair, P., Cousins, D. J., Louis, R., and Singh, D. (2021). Risankizumab in Severe Asthma - A Phase 2a, Placebo-Controlled Trial. N. Engl. J. Med. 385, 1669-1679. doi:10.1056/nejmoa2030880

Brightling, C. E., Gaga, M., Inoue, H., Li, J., Maspero, J., Wenzel, S., et al. (2021). Effectiveness of Fevipiprant in Reducing Exacerbations in Patients with Severe Asthma (LUSTER-1 and LUSTER-2): Two Phase 3 Randomised Controlled Trials. Lancet Respir. Med. 9, 43-56. doi:10.1016/S2213-2600(20)30412-4

Brusselle, G., Germinaro, M., Weiss, S., and Zangrilli, J. (2017). Reslizumab in Patients with Inadequately Controlled Late-Onset Asthma and Elevated Blood Eosinophils. Pulm. Pharmacol. Ther. 43, 39-45. doi:10.1016/j.pupt.2017.01.011

Busse, W., Corren, J., Lanier, B. Q., McAlary, M., Fowler-Taylor, A., Cioppa, G. D., et al. (2001). Omalizumab, Anti-IgE Recombinant Humanized Monoclonal Antibody, for the Treatment of Severe Allergic Asthma. J. Allergy Clin. Immunol. 108, 184-190. doi:10.1067/mai.2001.117880

Busse, W. W., Bleecker, E. R., FitzGerald, J. M., Ferguson, G. T., Barker, P., Sproule, S., et al. (2019). Long-term Safety and Efficacy of Benralizumab in Patients with Severe, Uncontrolled Asthma: 1-year Results from the BORA Phase 3 Extension Trial. Lancet Respir. Med. 7, 46-59. doi:10.1016/S2213-2600(18)30406-5

Castro, M., Corren, J., Pavord, I. D., Maspero, J., Wenzel, S., Rabe, K. F., et al. (2018). Dupilumab Efficacy and Safety in Moderate-To-Severe Uncontrolled Asthma. N. Engl. J. Med. 378, 2486-2496. doi:10.1056/NEJMoa1804092

Castro, M., Mathur, S., Hargreave, F., Boulet, L. P., Xie, F., Young, J., et al. (2011). Reslizumab for Poorly Controlled, Eosinophilic Asthma: a Randomized, Placebo-Controlled Study. Am. J. Respir. Crit. Care Med. 184, 1125-1132. doi:10.1164/rccm.201103-0396OC 
Castro, M., Zangrilli, J., Wechsler, M. E., Bateman, E. D., Brusselle, G. G., Bardin, P., et al. (2015). Reslizumab for Inadequately Controlled Asthma with Elevated Blood Eosinophil Counts: Results from Two Multicentre, Parallel, DoubleBlind, Randomised, Placebo-Controlled, Phase 3 Trials. Lancet Respir. Med. 3, 355-366. doi:10.1016/S2213-2600(15)00042-9

Castro, M., Kerwin, E., Miller, D., Pedinoff, A., Sher, L., Cardenas, P., et al. (2021). Efficacy and Safety of Fevipiprant in Patients with Uncontrolled Asthma: Two Replicate, Phase 3, Randomised, Double-Blind, Placebo-Controlled Trials (ZEAL-1 and ZEAL-2). EClinicalMedicine 35, 100847. doi:10.1016/ j.eclinm.2021.100847

Chan, Y. C., Ramadani, F., Santos, A. F., Pillai, P., Ohm-Laursen, L., Harper, C. E., et al. (2014). "Auto-anti-IgE": Naturally Occurring IgG Anti-IgE Antibodies May Inhibit Allergen-Induced Basophil Activation. J. Allergy Clin. Immunol. 134, 1394. doi:10.1016/j.jaci.2014.06.029

Chanez, P., Contin-Bordes, C., Garcia, G., Verkindre, C., Didier, A., De Blay, F., et al. (2010). Omalizumab-induced Decrease of FçRI Expression in Patients with Severe Allergic Asthma. Respir. Med. 104, 1608-1617. doi:10.1016/ j.rmed.2010.07.011

Chapman, K. R., Albers, F. C., Chipps, B., Muñoz, X., Devouassoux, G., Bergna, M., et al. (2019). The Clinical Benefit of Mepolizumab Replacing Omalizumab in Uncontrolled Severe Eosinophilic Asthma. Allergy 74, 1716-1726. doi:10.1111/ all.13850

Chen, Y. L., Gutowska-Owsiak, D., Hardman, C. S., Westmoreland, M., MacKenzie, T., Cifuentes, L., et al. (2019). Proof-of-concept Clinical Trial of Etokimab Shows a Key Role for IL-33 in Atopic Dermatitis Pathogenesis. Sci. Transl Med. 11, 11. doi:10.1126/scitranslmed.aax2945

Chinthrajah, S., Cao, S., Liu, C., Lyu, S. C., Sindher, S. B., Long, A., et al. (2019). Phase 2a Randomized, Placebo-Controlled Study of anti-IL-33 in Peanut Allergy. JCI Insight 4, 1-14. doi:10.1172/jci.insight.131347

Chupp, G., Lugogo, N. L., Kline, J. N., Ferguson, G. T., Hirsch, I., Goldman, M., et al. (2019). Rapid Onset of Effect of Benralizumab on Morning Peak Expiratory Flow in Severe, Uncontrolled Asthma. Ann. Allergy Asthma Immunol. 122, 478-485. doi:10.1016/j.anai.2019.02.016

Chupp, G. L., Bradford, E. S., Albers, F. C., Bratton, D. J., Wang-Jairaj, J., Nelsen, L. M., et al. (2017). Efficacy of Mepolizumab Add-On Therapy on Health-Related Quality of Life and Markers of Asthma Control in Severe Eosinophilic Asthma (MUSCA): a Randomised, Double-Blind, Placebo-Controlled, Parallel-Group, Multicentre, Phase 3b Trial. Lancet Respir. Med. 5, 390-400. doi:10.1016/S22132600(17)30125-X

Corne, J., Djukanovic, R., Thomas, L., Warner, J., Botta, L., Grandordy, B., et al. (1997). The Effect of Intravenous Administration of a Chimeric Anti-IgE Antibody on Serum IgE Levels in Atopic Subjects: Efficacy, Safety, and Pharmacokinetics. J. Clin. Invest. 99, 879-887. doi:10.1172/ JCI119252

Corren, J., Busse, W., Meltzer, E. O., Mansfield, L., Bensch, G., Fahrenholz, J., et al. (2010). A Randomized, Controlled, Phase 2 Study of AMG 317, an IL-4Ralpha Antagonist, in Patients with Asthma. Am. J. Respir. Crit. Care Med. 181, 788-796. doi:10.1164/rccm.200909-1448OC

Corren, J., Garcia Gil, E., Griffiths, J. M., Parnes, J. R., van der Merwe, R., Sałapa, K., et al. (2021). Tezepelumab Improves Patient-Reported Outcomes in Patients with Severe, Uncontrolled Asthma in PATHWAY. Ann. Allergy Asthma Immunol. 126, 187-193. doi:10.1016/j.anai.2020.10.008

Corren, J., Lemanske, R. F., Hanania, N. A., Korenblat, P. E., Parsey, M. V., Arron, J. R., et al. (2011). Lebrikizumab Treatment in Adults with Asthma. N. Engl. J. Med. 365, 1088-1098. doi:10.1056/NEJMoa1106469

Corren, J., Parnes, J. R., Wang, L., Mo, M., Roseti, S. L., Griffiths, J. M., et al. (2017). Tezepelumab in Adults with Uncontrolled Asthma. N. Engl. J. Med. 377, 936-946. doi:10.1056/nejmoa1704064

Corren, J., Weinstein, S., Janka, L., Zangrilli, J., and Garin, M. (2016). Phase 3 Study of Reslizumab in Patients with Poorly Controlled Asthma: Effects across a Broad Range of Eosinophil Counts. Chest 150, 799-810. doi:10.1016/ j.chest.2016.03.018

Corren, J., Karpefors, M., Hellqvist, A., Parnes, J. R., and Colice, G. (2021). Tezepelumab Reduces Exacerbations across All Seasons in Patients with Severe, Uncontrolled Asthma: A Post Hoc Analysis of the PATHWAY Phase 2b Study. Jaa 14, 1-11. doi:10.2147/JAA.S286036

d'Ancona, G., Kavanagh, J., Roxas, C., Green, L., Fernandes, M., Thomson, L., et al. (2020). Adherence to Corticosteroids and Clinical Outcomes in Mepolizumab
Therapy for Severe Asthma. Eur. Respir. J. 55, 1902259. doi:10.1183/ 13993003.02259-2019

De Boever, E. H., Ashman, C., Cahn, A. P., Locantore, N. W., Overend, P., Pouliquen, I. J., et al. (2014). Efficacy and Safety of an anti-IL-13 mAb in Patients with Severe Asthma: a Randomized Trial. J. Allergy Clin. Immunol. 133, 989-996. doi:10.1016/j.jaci.2014.01.002

de Llano, L. P., Venneradel, Mdel. C. C., Álvarez, F. J., Medina, J. F., Borderías, L., Pellicer, C., et al. (2013). Effects of Omalizumab in Non-atopic Asthma: Results from a Spanish Multicenter Registry. J. Asthma 50, 296-301. doi:10.3109/ 02770903.2012.757780

De Vries, R., Dagelet, Y. W. F., Spoor, P., Snoey, E., Jak, P. M. C., Brinkman, P., et al. (2018). Clinical and Inflammatory Phenotyping by Breathomics in Chronic Airway Diseases Irrespective of the Diagnostic Label. Eur. Respir. J. 51, 1-10. doi:10.1183/13993003.01817-2017

Diver, S., Khalfaoui, L., Emson, C., Wenzel, S. E., Menzies-Gow, A., Wechsler, M. E., et al. (2021). Effect of Tezepelumab on Airway Inflammatory Cells, Remodelling, and Hyperresponsiveness in Patients with Moderate-To-Severe Uncontrolled Asthma (CASCADE): a Double-Blind, Randomised, PlaceboControlled, Phase 2 Trial. Lancet Respir. Med. 11, 1299-1312. doi:10.1016/ S2213-2600(21)00226-5

Djukanović, R., Wilson, S. J., Kraft, M., Jarjour, N. N., Steel, M., Chung, K. F., et al. (2004). Effects of Treatment with Anti-immunoglobulin E Antibody Omalizumab on Airway Inflammation in Allergic Asthma. Am. J. Respir. Crit. Care Med. 170, 583-593. doi:10.1164/rccm.200312-1651OC

Drick, N., Seeliger, B., Welte, T., Fuge, J., and Suhling, H. (2018). Anti-IL-5 Therapy in Patients with Severe Eosinophilic Asthma - Clinical Efficacy and Possible Criteria for Treatment Response. BMC Pulm. Med. 18, 119. doi:10.1186/ s12890-018-0689-2

Duncan, A. R., and Winter, G. (1988). The Binding Site for C1q on IgG. Nature 332, 738-740. doi:10.1038/332738a0

Dunican, E. M., Elicker, B. M., Gierada, D. S., Nagle, S. K., Schiebler, M. L., Newell, J. D., et al. (2018). Mucus Plugs in Patients with Asthma Linked to Eosinophilia and Airflow Obstruction. J. Clin. Invest. 128, 997-1009. doi:10.1172/JCI95693

Eck, S., Castro, M., Sinibaldi, D., White, W., Folliot, K., Gossage, D., et al. (2014). Benralizumab Effect on Blood Basophil Counts in Adults with Uncontrolled Asthma. Eur. Respir. J. 44, 297, 2014 . Available at: http://erj.ersjournals.com/ content/44/Suppl_58/P297.abstract.

Eger, K., Pet, L., Weersink, E. J. M., and Bel, E. H. (2021). Complications of Switching from anti-IL-5 or anti-IL-5R to Dupilumab in Corticosteroiddependent Severe Asthma. J. Allergy Clin. Immunol. Pract. 9, 2913-2915. doi:10.1016/j.jaip.2021.02.042

Elliott, E. I., and Sutterwala, F. S. (2015). Initiation and Perpetuation of NLRP3 Inflammasome Activation and Assembly. Immunol. Rev. 265, 35-52. doi:10.1111/imr.12286

Erpenbeck, V. J., Popov, T. A., Miller, D., Weinstein, S. F., Spector, S., Magnusson, B., et al. (2016). The Oral CRTh2 Antagonist QAW039 (Fevipiprant): A Phase II Study in Uncontrolled Allergic Asthma. Pulm. Pharmacol. Ther. 39, 54-63. doi:10.1016/j.pupt.2016.06.005

Fahy, J. V., Fleming, H. E., Wong, H. H., Liu, J. T., Su, J. Q., Reimann, J., et al. (1997). The Effect of an Anti-IgE Monoclonal Antibody on the Early- and Latephase Responses to Allergen Inhalation in Asthmatic Subjects. Am. J. Respir. Crit. Care Med. 155, 1828-1834. doi:10.1164/ajrccm.155.6.9196082

Ferguson, G. T., FitzGerald, J. M., Bleecker, E. R., Laviolette, M., Bernstein, D., LaForce, C., et al. (2017). Benralizumab for Patients with Mild to Moderate, Persistent Asthma (BISE): a Randomised, Double-Blind, Placebo-Controlled, Phase 3 Trial. Lancet Respir. Med. 5, 568-576. doi:10.1016/S2213-2600(17)30190-X

FitzGerald, J. M., Bleecker, E. R., Nair, P., Korn, S., Ohta, K., Lommatzsch, M., et al. (2016). Benralizumab, an Anti-interleukin-5 Receptor a Monoclonal Antibody, as Add-On Treatment for Patients with Severe, Uncontrolled, Eosinophilic Asthma (CALIMA): a Randomised, Double-Blind, Placebo-Controlled Phase 3 Trial. Lancet 388, 2128-2141. doi:10.1016/S0140-6736(16)31322-8

Flood-Page, P. T., Menzies-Gow, A. N., Kay, A. B., and Robinson, D. S. (2003). Eosinophil's Role Remains Uncertain as Anti-interleukin-5 Only Partially Depletes Numbers in Asthmatic Airway. Am. J. Respir. Crit. Care Med. 167, 199-204. doi:10.1164/rccm.200208-789OC

Gamble, J., Stevenson, M., McClean, E., and Heaney, L. G. (2009). The Prevalence of Nonadherence in Difficult Asthma. Am. J. Respir. Crit. Care Med. 180, 817-822. doi:10.1164/rccm.200902-0166OC 
Garcia, G., Magnan, A., Chiron, R., Contin-Bordes, C., Berger, P., Taillé, C., et al. (2013). A Proof-Of-Concept, Randomized, Controlled Trial of Omalizumab in Patients with Severe, Difficult-To-Control, Nonatopic Asthma. Chest 144, 411-419. doi:10.1378/chest.12-1961

Gauvreau, G. M., O’Byrne, P. M., Boulet, L. P., Wang, Y., Cockcroft, D., Bigler, J., et al. (2014). Effects of an Anti-TSLP Antibody on Allergen-Induced Asthmatic Responses. N. Engl. J. Med. 370, 2102-2110. doi:10.1056/NEJMoa1402895

Gauvreau, G., Sehmi, R., FitzGerald, J., Leigh, R., Cockcroft, D., Davis, B., et al. (2021). The Effect of Benralizumab on Allergen-Induced Responses in Subjects with Mild Allergic Asthma. J. Allergy Clin. Immunol. 147, AB157. doi:10.1016/ j.jaci.2020.12.563

Gonem, S., Berair, R., Singapuri, A., Hartley, R., Laurencin, M. F. M., Bacher, G., et al. (2016). Fevipiprant, a Prostaglandin D2 Receptor 2 Antagonist, in Patients with Persistent Eosinophilic Asthma: a single-centre, Randomised, DoubleBlind, Parallel-Group, Placebo-Controlled Trial. Lancet Respir. Med. 4, 699-707. doi:10.1016/S2213-2600(16)30179-5

Gour, N., and Wills-Karp, M. (2015). IL-4 and IL-13 Signaling in Allergic Airway Disease. Cytokine 75, 68-78. doi:10.1016/j.cyto.2015.05.014

Haldar, P., Brightling, C. E., Hargadon, B., Gupta, S., Monteiro, W., Sousa, A., et al. (2009). Mepolizumab and Exacerbations of Refractory Eosinophilic Asthma. N. Engl. J. Med. 360, 973-984. doi:10.1056/NEJMoa0808991

Hammad, H., and Lambrecht, B. N. (2021). The Basic Immunology of Asthma. Cell 184, 1469-1485. doi:10.1016/j.cell.2021.02.016

Hanania, N. A., Alpan, O., Hamilos, D. L., Condemi, J. J., Reyes-Rivera, I., Zhu, J., et al. (2011). Omalizumab in Severe Allergic Asthma Inadequately Controlled with Standard Therapy: a Randomized Trial. Ann. Intern. Med. 154, 573-582. doi:10.7326/0003-4819-154-9-201105030-00002

Hanania, N. A., Korenblat, P., Chapman, K. R., Bateman, E. D., Kopecky, P., Paggiaro, P., et al. (2016). Efficacy and Safety of Lebrikizumab in Patients with Uncontrolled Asthma (LAVOLTA I and LAVOLTA II): Replicate, Phase 3, Randomised, Double-Blind, Placebo-Controlled Trials. Lancet Respir. Med. 4, 781-796. doi:10.1016/S2213-2600(16)30265-X

Hanania, N. A., Noonan, M., Corren, J., Korenblat, P., Zheng, Y., Fischer, S. K., et al. (2015). Lebrikizumab in Moderate-To-Severe Asthma: Pooled Data from Two Randomised Placebo-Controlled Studies. Thorax 70, 748-756. doi:10.1136/thoraxjnl-2014-206719

Hanania, N. A., Wenzel, S., Rosén, K., Hsieh, H. J., Mosesova, S., Choy, D. F., et al. (2013). Exploring the Effects of Omalizumab in Allergic Asthma: an Analysis of Biomarkers in the EXTRA Study. Am. J. Respir. Crit. Care Med. 187, 804-811. doi:10.1164/rccm.201208-1414OC

Harrison, T., Canonica, G. W., Chupp, G., Lee, J., Schleich, F., Welte, T., et al. (2020). Real-world Mepolizumab in the Prospective Severe Asthma REALITI-A Study: Initial Analysis. Eur. Respir. J. 56, 56. doi:10.1183/ 13993003.00151-2020

Hart, T. K., Blackburn, M. N., Brigham-Burke, M., Dede, K., Al-Mahdi, N., ZiaAmirhosseini, P., et al. (2002). Preclinical Efficacy and Safety of Pascolizumab (SB 240683): a Humanized Anti-interleukin-4 Antibody with Therapeutic Potential in Asthma. Clin. Exp. Immunol. 130, 93-100. doi:10.1046/j.13652249.2002.01973.x

Heffler, E., Blasi, F., Latorre, M., Menzella, F., Paggiaro, P., Pelaia, G., et al. (2019). The Severe Asthma Network in Italy: Findings and Perspectives. J. Allergy Clin. Immunol. Pract. 7, 1462-1468. doi:10.1016/j.jaip.2018.10.016

Henault, J., Riggs, J. M., Karnell, J. L., Liarski, V. M., Li, J., Shirinian, L., et al. (2016). Self-reactive IgE Exacerbates Interferon Responses Associated with Autoimmunity. Nat. Immunol. 17, 196-203. doi:10.1038/ni.3326

Hirano, I., Peterson, K., Murray, J., Dellon, E., Falk, G., Gonsalves, N., et al. (2020). AK002, an Anti-siglec-8 Antibody, Depletes Tissue Eosinophils and Improves Dysphagia Symptoms in Patients with Eosinophilic Esophagitis. J. Allergy Clin. Immunol. 145, AB167. doi:10.1016/j.jaci.2019.12.343

Ho, T., Al-Selahi, E., Mukherjee, M., Huang, C., Radford, K., Kjarsgaard, M., et al. (2020). Sputum and Serum Immunoglobulins in Adult Asthmatics with Recurrent Respiratory Tract Infections. Allergy 75, 2105-2108. doi:10.1111/ all.14283

Hodsman, P., Ashman, C., Cahn, A., De Boever, E., Locantore, N., Serone, A., et al. (2013). A Phase 1, Randomized, Placebo-Controlled, Dose-Escalation Study of an anti-IL-13 Monoclonal Antibody in Healthy Subjects and Mild Asthmatics. Br. J. Clin. Pharmacol. 75, 118-128. doi:10.1111/j.1365-2125.2012.04334.x
Hogan, S. P., Rosenberg, H. F., Moqbel, R., Phipps, S., Foster, P. S., Lacy, P., et al. (2008). Eosinophils: Biological Properties and Role in Health and Disease. Clin. Exp. Allergy 38, 709-750. doi:10.1111/j.13652222.2008.02958.x

Holgate, S., Casale, T., Wenzel, S., Bousquet, J., Deniz, Y., and Reisner, C. (2005). The Anti-inflammatory Effects of Omalizumab Confirm the central Role of IgE in Allergic Inflammation. J. Allergy Clin. Immunol. 115, 459-465. doi:10.1016/ j.jaci.2004.11.053

Humbert, M., Beasley, R., Ayres, J., Slavin, R., Hébert, J., Bousquet, J., et al. (2005). Benefits of Omalizumab as Add-On Therapy in Patients with Severe Persistent Asthma Who Are Inadequately Controlled Despite Best Available Therapy (GINA 2002 Step 4 Treatment): INNOVATE. Allergy 60, 309-316. doi:10.1111/ j.1398-9995.2004.00772.x

Ibrahim, H., O’Sullivan, R., Casey, D., Murphy, J., MacSharry, J., Plant, B. J., et al. (2019). The Effectiveness of Reslizumab in Severe Asthma Treatment: a RealWorld Experience. Respir. Res. 20, 289. doi:10.1186/s12931-019-1251-3

Izumo, T., Tone, M., Kuse, N., Awano, N., Tanaka, A., Jo, T., et al. (2020). Effectiveness and Safety of Benralizumab for Severe Asthma in Clinical Practice (J-BEST): a Prospective Study. Ann. Transl Med. 8, 438. doi:10.21037/ atm.2020.04.01

Johanson, K., Appelbaum, E., Doyle, M., Hensley, P., Zhao, B., Abdel-Meguid, S. S., et al. (1995). Binding Interactions of Human Interleukin 5 with its Receptor a Subunit. J. Biol. Chem. 270, 9459-9471. doi:10.1074/jbc.270.16.9459

Kavanagh, J. E., Hearn, A. P., Dhariwal, J., d'Ancona, G., Douiri, A., Roxas, C., et al. (2021). Real-World Effectiveness of Benralizumab in Severe Eosinophilic Asthma. Chest 159, 496-506. doi:10.1016/j.chest.2020.08.2083

Khatri, S., Moore, W., Gibson, P. G., Leigh, R., Bourdin, A., Maspero, J., et al. (2019). Assessment of the Long-Term Safety of Mepolizumab and Durability of Clinical Response in Patients with Severe Eosinophilic Asthma. J. Allergy Clin. Immunol. 143, 1742. doi:10.1016/j.jaci.2018.09.033

Khurana, S., Brusselle, G. G., Bel, E. H., FitzGerald, J. M., Masoli, M., Korn, S., et al. (2019). Long-term Safety and Clinical Benefit of Mepolizumab in Patients with the Most Severe Eosinophilic Asthma: The COSMEX Study. Clin. Ther. 41, 2041. doi:10.1016/j.clinthera.2019.07.007

Kim, R. Y., Pinkerton, J. W., Essilfie, A. T., Robertson, A. A. B., Baines, K. J., Brown, A. C., et al. (2017). Role for NLRP3 Inflammasome-Mediated, IL-1 $\beta$-Dependent Responses in Severe, Steroid-Resistant Asthma. Am. J. Respir. Crit. Care Med. 196, 283-297. doi:10.1164/rccm.201609-1830OC

Kips, J. C., O’Connor, B. J., Langley, S. J., Woodcock, A., Kerstjens, H. A., Postma, D. S., et al. (2003). Effect of SCH55700, a Humanized Anti-human Interleukin5 Antibody, in Severe Persistent Asthma: a Pilot Study. Am. J. Respir. Crit. Care Med. 167, 1655-1659. doi:10.1164/rccm.200206-525OC

Korenblat, P., Kerwin, E., Leshchenko, I., Yen, K., Holweg, C. T. J., AnzuresCabrera, J., et al. (2018). Efficacy and Safety of Lebrikizumab in Adult Patients with Mild-To-Moderate Asthma Not Receiving Inhaled Corticosteroids. Respir. Med. 134, 143-149. doi:10.1016/j.rmed.2017.12.006

Laidlaw, T. M., Prussin, C., Panettieri, R. A., Lee, S., Ferguson, B. J., Adappa, N. D., et al. (2019). Dexpramipexole Depletes Blood and Tissue Eosinophils in Nasal Polyps with No Change in Polyp Size. Laryngoscope 129, E61-E66. doi:10.1002/ lary. 27564

Leckie, M. J., ten Brinke, A., Khan, J., Diamant, Z., O’Connor, B. J., Walls, C. M., et al. (2000). Effects of an Interleukin-5 Blocking Monoclonal Antibody on Eosinophils, Airway Hyper-Responsiveness, and the Late Asthmatic Response. Lancet 356, 2144-2148. doi:10.1016/s0140-6736(00)03496-6

Lee, J., Tay, T. R., Radhakrishna, N., Hore-Lacy, F., Mackay, A., Hoy, R., et al. (2018). Nonadherence in the Era of Severe Asthma Biologics and Thermoplasty. Eur. Respir. J. 51, 51. doi:10.1183/13993003.01836-2017

Levine, H. T., Tauber, J., Nguyen, Q., Anesi, S., Chang, P., Berdy, G., et al. (2020). Phase $1 \mathrm{~b}$ Study of AK002, an Anti-siglec-8 Monoclonal Antibody, in Patients with Severe Allergic Conjunctivitis (KRONOS Study). J. Allergy Clin. Immunol. 145, AB185. doi:10.1016/j.jaci.2019.12.323

Li, Y., and Hua, S. (2014). Mechanisms of Pathogenesis in Allergic Asthma: Role of Interleukin-23. Respirology 19, 663-669. doi:10.1111/resp.12299

Liu, S., Verma, M., Michalec, L., Liu, W., Sripada, A., Rollins, D., et al. (2018). Steroid Resistance of Airway Type 2 Innate Lymphoid Cells from Patients with Severe Asthma: The Role of Thymic Stromal Lymphopoietin. J. Allergy Clin. Immunol. 141, 257. doi:10.1016/j.jaci.2017.03.032 
Lugogo, N., Domingo, C., Chanez, P., Leigh, R., Gilson, M. J., Price, R. G., et al. (2016). Long-term Efficacy and Safety of Mepolizumab in Patients with Severe Eosinophilic Asthma: A Multi-center, Open-Label, Phase IIIb Study. Clin. Ther. 38, 2058. doi:10.1016/j.clinthera.2016.07.010

Machida, K., Aw, M., Salter, B. M. A., Ju, X., Mukherjee, M., Gauvreau, G. M., et al. (2020). The Role of the TL1A/DR3 Axis in the Activation of Group 2 Innate Lymphoid Cells in Subjects with Eosinophilic Asthma. Am. J. Respir. Crit. Care Med. 202, 1105-1114. doi:10.1164/rccm.201909-1722OC

Marone, G., Spadaro, G., Braile, M., Poto, R., Criscuolo, G., Pahima, H., et al. (2019). Tezepelumab: a Novel Biological Therapy for the Treatment of Severe Uncontrolled Asthma. Expert Opin. Investig. Drugs 28, 931-940. doi:10.1080/ 13543784.2019.1672657

Máspero, J. (2017). Reslizumab in the Treatment of Inadequately Controlled Asthma in Adults and Adolescents with Elevated Blood Eosinophils: Clinical Trial Evidence and Future Prospects. Ther. Adv. Respir. Dis. 11, 311-325. doi:10.1177/1753465817717134

Maspero, J. F., Katelaris, C. H., Busse, W. W., Castro, M., Corren, J., Chipps, B. E., et al. (2020). Dupilumab Efficacy in Uncontrolled, Moderate-To-Severe Asthma with Self-Reported Chronic Rhinosinusitis. J. Allergy Clin. Immunol. Pract. 8, 527. doi:10.1016/j.jaip.2019.07.016

Menzies-Gow, A., Corren, J., Bel, E. H., Maspero, J., Heaney, L. G., Gurnell, M., et al. (2019). Corticosteroid Tapering with Benralizumab Treatment for Eosinophilic Asthma: PONENTE Trial. ERJ Open Res. 5, 00009-02019. doi:10.1183/23120541.00009-2019

Menzies-Gow, A., Corren, J., Bourdin, A., Chupp, G., Israel, E., Wechsler, M. E., et al. (2021). Tezepelumab in Adults and Adolescents with Severe, Uncontrolled Asthma. N. Engl. J. Med. 384, 1800-1809. doi:10.1056/nejmoa2034975

Menzies-Gow, A., Ponnarambil, S., Downie, J., Bowen, K., Hellqvist, A., and Colice, G. (2020). DESTINATION: a Phase 3, Multicentre, Randomized, Double-Blind, Placebo-Controlled, Parallel-Group Trial to Evaluate the Long-Term Safety and Tolerability of Tezepelumab in Adults and Adolescents with Severe, Uncontrolled Asthma. Respir. Res. 21, 279. doi:10.1186/s12931-020-01541-7

Mukherjee, M., Aleman Paramo, F., Kjarsgaard, M., Salter, B., Nair, G., LaVigne, N., et al. (2018). Weight-adjusted Intravenous Reslizumab in Severe Asthma with Inadequate Response to Fixed-Dose Subcutaneous Mepolizumab. Am. J. Respir. Crit. Care Med. 197, 38-46. doi:10.1164/rccm.201707-1323OC

Mukherjee, M., Kjarsgaard, M., Radford, K., Huang, C., Leigh, R., Dorscheid, D. R., et al. (2019). Omalizumab in Patients With Severe Asthma and Persistent Sputum Eosinophilia. Allergy Asthma Clin. Immunol. 15, 21. doi:10.1186/ s13223-019-0337-2

Mukherjee, M., Forero, D. F., Tran, S., Boulay, M. E., Bertrand, M., Bhalla, A., et al. (2020). Suboptimal Treatment Response to anti-IL-5 Monoclonal Antibodies in Severe Eosinophilic Asthmatics with Airway Autoimmune Phenomena. Eur. Respir. J. 56 (4), 2000117. doi:10.1183/13993003.00117-2020

Mukherjee, M., Lacy, P., and Ueki, S. (2018). Eosinophil Extracellular Traps and Inflammatory Pathologies-Untangling the Web!. Front. Immunol. 9, 2763. doi:10.3389/fimmu.2018.02763

Mukherjee, M., Lim, H. F., Thomas, S., Miller, D., Kjarsgaard, M., Tan, B., et al. (2017). Airway Autoimmune Responses in Severe Eosinophilic Asthma Following Low-Dose Mepolizumab Therapy. Allergy Asthma Clin. Immunol. 13, 2. doi:10.1186/s13223-016-0174-5

Mukherjee, M., and Nair, P. (2018). Autoimmune Responses in Severe Asthma. Allergy Asthma Immunol. Res. 10, 428-447. doi:10.4168/ aair.2018.10.5.428

Mukherjee, M., and Nair, P. (2015). Blood or Sputum Eosinophils to Guide Asthma Therapy? Lancet Respir. Med. 3, 824-825. doi:10.1016/S2213-2600(15)00419-1

Munitz, A., Brandt, E. B., Mingler, M., Finkelman, F. D., and Rothenberg, M. E. (2008). Distinct Roles for IL-13 and IL-4 via IL-13 Receptor Alphal and the Type II IL-4 Receptor in Asthma Pathogenesis. Proc. Natl. Acad. Sci. U S A. 105, 7240-7245. doi:10.1073/pnas.0802465105

Murphy, K., Jacobs, J., Bjermer, L., Fahrenholz, J. M., Shalit, Y., Garin, M., et al. (2017). Long-term Safety and Efficacy of Reslizumab in Patients with Eosinophilic Asthma. J. Allergy Clin. Immunol. Pract. 5, 1572. doi:10.1016/ j.jaip.2017.08.024

Nair, P., Pizzichini, M. M., Kjarsgaard, M., Inman, M. D., Efthimiadis, A., Pizzichini, E., et al. (2009). Mepolizumab for Prednisone-dependent Asthma with Sputum Eosinophilia. N. Engl. J. Med. 360, 985-993. doi:10.1056/ NEJMoa0805435
Nair, P., Wenzel, S., Rabe, K. F., Bourdin, A., Lugogo, N. L., Kuna, P., et al. (2017) Oral Glucocorticoid-Sparing Effect of Benralizumab in Severe Asthma. N. Engl. J. Med. 376, 2448-2458. doi:10.1056/NEJMoa1703501

Noonan, M., Korenblat, P., Mosesova, S., Scheerens, H., Arron, J. R., Zheng, Y., et al. (2013). Dose-ranging Study of Lebrikizumab in Asthmatic Patients Not Receiving Inhaled Steroids. J. Allergy Clin. Immunol. 132, 567. doi:10.1016/ j.jaci.2013.03.051

Normansell, R., Walker, S., Milan, S. J., Walters, E. H., and Nair, P. (2014). Omalizumab for Asthma in Adults and Children. Cochrane Database Syst. Rev. 13 (1), CD003559 doi:10.1002/14651858.CD003559.pub4

Nowak, R. M., Parker, J. M., Silverman, R. A., Rowe, B. H., Smithline, H., Khan, F., et al. (2015). A Randomized Trial of Benralizumab, an Antiinterleukin 5 Receptor a Monoclonal Antibody, after Acute Asthma. Am. J. Emerg. Med. 33, 14-20. doi:10.1016/j.ajem.2014.09.036

Ojanguren, I., Chaboillez, S., and Lemiere, C. (2018). Low Blood Eosinophil Counts Are Not Always a Reliable Marker of Clinical Response to Mepolizumab in Severe Asthma. J. Allergy Clin. Immunol. Pract. 6, 2151-2153. doi:10.1016/ j.jaip.2018.04.014

Ortega, H. G., Liu, M. C., Pavord, I. D., Brusselle, G. G., FitzGerald, J. M., Chetta, A., et al. (2014). Mepolizumab Treatment in Patients with Severe Eosinophilic Asthma. N. Engl. J. Med. 371, 1198-1207. doi:10.1056/NEJMoa1403290

Ortega, H. G., Yancey, S. W., Mayer, B., Gunsoy, N. B., Keene, O. N., Bleecker, E. R., et al. (2016). Severe Eosinophilic Asthma Treated with Mepolizumab Stratified by Baseline Eosinophil Thresholds: a Secondary Analysis of the DREAM and MENSA Studies. Lancet Respir. Med. 4, 549-556. doi:10.1016/S2213-2600(16) 30031-5

Otulana, B. A., Wenzel, S. E., Ind, P. W., Bowden, A., Puthukkeril, S., Tomkinson, A., et al. (2011). "A Phase $2 b$ Study of Inhaled Pitrakinra, an IL-4/IL-13 Antagonist, Successfully Identified Responder Subpopulations of Patients with Uncontrolled Asthma," in D101. ASTHMA GENETICS American Thoracic Society International Conference Abstracts. (American Thoracic Society), Denver, CO, A6179. doi:10.1164/ajrccmconference.2011.183.1_MeetingAbstracts.A6179

Panettieri, R. A., Jr, Sjöbring, U., Péterffy, A., Wessman, P., Bowen, K., Piper, E., et al. (2018). Tralokinumab for Severe, Uncontrolled Asthma (STRATOS 1 and STRATOS 2): Two Randomised, Double-Blind, Placebo-Controlled, Phase 3 Clinical Trials. Lancet Respir. Med. 6, 511-525. doi:10.1016/S2213-2600(18) 30184-X

Pavord, I. D., Korn, S., Howarth, P., Bleecker, E. R., Buhl, R., Keene, O. N., et al. (2012). Mepolizumab for Severe Eosinophilic Asthma (DREAM): a Multicentre, Double-Blind, Placebo-Controlled Trial. Lancet 380, 651-659. doi:10.1016/S0140-6736(12)60988-X

Pazdrak, K., Stafford, S., and Alam, R. (1995). The Activation of the Jak-STAT 1 Signaling Pathway by IL-5 in Eosinophils. J. Immunol. 155, 397-402. Available at: http://www.jimmunol.org/content/155/1/397.abstract.

Pelaia, C., Busceti, M. T., Solinas, S., Terracciano, R., and Pelaia, G. (2018). Real-life Evaluation of the Clinical, Functional, and Hematological Effects of Mepolizumab in Patients with Severe Eosinophilic Asthma: Results of a Single-centre Observational Study. Pulm. Pharmacol. Ther. 53, 1-5. doi:10.1016/j.pupt.2018.09.006

Pelaia, C., Calabrese, C., Terracciano, R., de Blasio, F., Vatrella, A., and Pelaia, G. (2018). Omalizumab, the First Available Antibody for Biological Treatment of Severe Asthma: More Than a Decade of Real-Life Effectiveness. Ther. Adv. Respir. Dis. 12, 1753466618810192. doi:10.1177/1753466618810192

Pérez de Llano, L. A., Cosío, B. G., Domingo, C., Urrutia, I., Bobolea, I., Valero, A., et al. (2019). Efficacy and Safety of Reslizumab in Patients with Severe Asthma with Inadequate Response to Omalizumab: A Multicenter, Open-Label Pilot Study. J. Allergy Clin. Immunol. Pract. 7, 2277. doi:10.1016/j.jaip.2019.01.017

Piper, E., Brightling, C., Niven, R., Oh, C., Faggioni, R., Poon, K., et al. (2013). A Phase II Placebo-Controlled Study of Tralokinumab in Moderate-To-Severe Asthma. Eur. Respir. J. 41, 330-338. doi:10.1183/09031936.00223411

Poznanski, S. M., Mukherjee, M., Zhao, N., Huang, C., Radford, K., Ashkar, A. A., et al. (2021). Asthma Exacerbations on Benralizumab Are Largely Noneosinophilic. Allergy 76, 375-379. doi:10.1111/all.14514

Prussin, C., Laidlaw, T. M., Panettieri, R. A., Ferguson, B. J., Adappa, N. D., Lane, A. P., et al. (2017). Dexpramipexole Effectively Lowers Blood and Tissue Eosinophils in Subjects with Chronic Rhinosinusitis with Nasal Polyps. J. Allergy Clin. Immunol. 139 (2), AB64. doi:10.1016/j.jaci.2016.12.256 
Prussin, C., Panettieri, R. A., Bozik, M. E., Archibald, D. G., Mather, J. L., and Siddiqui, S. (2021). Oral Dexpramipexole Efficacy in Lowering Blood Eosinophils in Patients with Moderate to Severe Uncontrolled Eosinophilic Asthma: Study Design and Baseline Data from the AS201 Phase 2 Trial, A1359. doi:10.1164/ajrccm-conference.2021.203.1_meetingabstracts.a1359

Rabe, K. F., Nair, P., Maspero, J. F., Castro, M., Rice, M. S., Deniz, Y., et al. (2020). The Effect of Dupilumab on Lung Function Parameters in Patients with Oral Corticosteroid-dependent Severe Asthma. Respir. Med. X 2, 100010. doi:10.1016/j.yrmex.2019.100010

Ray, A., Raundhal, M., Oriss, T. B., Ray, P., and Wenzel, S. E. (2016). Current Concepts of Severe Asthma. J. Clin. Invest. 126, 2394-2403. doi:10.1172/ JCI84144

Ricciardolo, F. L. M., Sorbello, V., Folino, A., Gallo, F., Massaglia, G. M., Favatà, G., et al. (2017). Identification of IL-17F/frequent Exacerbator Endotype in Asthma. J. Allergy Clin. Immunol. 140, 395-406. doi:10.1016/j.jaci.2016.10.034

Rodrigo, G. J., Neffen, H., and Castro-Rodriguez, J. A. (2011). Efficacy and Safety of Subcutaneous Omalizumab vs Placebo as Add-On Therapy to Corticosteroids for Children and Adults with Asthma: a Systematic Review. Chest 139, 28-35. doi:10.1378/chest.10-1194

Rossjohn, J., McKinstry, W. J., Woodcock, J. M., McClure, B. J., Hercus, T. R., Parker, M. W., et al. (2000). Structure of the Activation Domain of the GMCSF/IL-3/IL-5 Receptor Common Beta-Chain Bound to an Antagonist. Blood 95, 2491-2498. doi:10.1182/blood.V95.8.2491

Russell, R. J., Chachi, L., FitzGerald, J. M., Backer, V., Olivenstein, R., Titlestad, I. L., et al. (2018). Effect of Tralokinumab, an Interleukin-13 Neutralising Monoclonal Antibody, on Eosinophilic Airway Inflammation in Uncontrolled Moderate-To-Severe Asthma (MESOS): a Multicentre, Double-Blind, Randomised, Placebo-Controlled Phase 2 Trial. Lancet Respir. Med. 6, 499-510. doi:10.1016/S2213-2600(18)30201-7

Salter, B. M., Aw, M., and Sehmi, R. (2019). The Role of Type 2 Innate Lymphoid Cells in Eosinophilic Asthma. J. Leukoc. Biol. 106, 889-901. doi:10.1002/ JLB.3MR1217-497R

Salter, B. M., Oliveria, J. P., Nusca, G., Smith, S. G., Tworek, D., Mitchell, P. D., et al. (2016). IL-25 and IL-33 Induce Type 2 Inflammation in Basophils from Subjects with Allergic Asthma. Respir. Res. 17, 5. doi:10.1186/s12931-0160321-z

Salter, B. M., Oliveria, J. P., Nusca, G., Smith, S. G., Watson, R. M., Comeau, M., et al. (2015). Thymic Stromal Lymphopoietin Activation of Basophils in Patients with Allergic Asthma Is IL-3 Dependent. J. Allergy Clin. Immunol. 136, 1636-1644. doi:10.1016/j.jaci.2015.03.039

Sano, M., Leff, A. R., Myou, S., Boetticher, E., Meliton, A. Y., Learoyd, J., et al. (2005). Regulation of Interleukin-5-Induced Beta2-Integrin Adhesion of Human Eosinophils by Phosphoinositide 3-kinase. Am. J. Respir. Cel Mol Biol 33, 65-70. doi:10.1165/rcmb.2005-0076OC

Saunders, R., Kaul, H., Berair, R., Gonem, S., Singapuri, A., Sutcliffe, A. J., et al. (2019). DP2 Antagonism Reduces Airway Smooth Muscle Mass in Asthma by Decreasing Eosinophilia and Myofibroblast Recruitment. Sci. Transl Med. 11, 1-12. doi:10.1126/scitranslmed.aao6451

Schumann, C., Kropf, C., Wibmer, T., Rüdiger, S., Stoiber, K. M., Thielen, A., et al. (2012). Omalizumab in Patients with Severe Asthma: the XCLUSIVE Study. Clin. Respir. J. 6, 215-227. doi:10.1111/j.1752-699X.2011.00263.x

Schwartz, C., Willebrand, R., Huber, S., Rupec, R. A., Wu, D., Locksley, R., et al. (2015). Eosinophil-specific Deletion of IkBa in Mice Reveals a Critical Role of NF-Kb-Induced Bcl-xL for Inhibition of Apoptosis. Blood 125, 3896-3904. doi:10.1182/blood-2014-10-607788

Sehmi, R., Lim, H. F., Mukherjee, M., Huang, C., Radford, K., Newbold, P., et al. (2018). Benralizumab Attenuates Airway Eosinophilia in Prednisonedependent Asthma. J. Allergy Clin. Immunol. 141, 1529. doi:10.1016/ j.jaci.2018.01.008

Simpson, J. L., Phipps, S., Baines, K. J., Oreo, K. M., Gunawardhana, L., and Gibson, P. G. (2014). Elevated Expression of the NLRP3 Inflammasome in Neutrophilic Asthma. Eur. Respir. J. 43, 1067-1076. doi:10.1183/09031936.00105013

Smith, S. G., Chen, R., Kjarsgaard, M., Huang, C., Oliveria, J. P., O’Byrne, P. M., et al. (2016). Increased Numbers of Activated Group 2 Innate Lymphoid Cells in the Airways of Patients with Severe Asthma and Persistent Airway Eosinophilia. J. Allergy Clin. Immunol. 137, 75. doi:10.1016/j.jaci.2015.05.037
Sterk, P. J. (2019). Modern Inflammatory Phenotyping of Asthma. Breathomics Is Here to Stay. Am. J. Respir. Crit. Care Med. 200, 405-406. doi:10.1164/ rccm.201904-0733ED

Stokol, T., O'Donnell, P., Xiao, L., Knight, S., Stavrakis, G., Botto, M., et al. (2004). C1q Governs Deposition of Circulating Immune Complexes and Leukocyte Fc $\gamma$ Receptors Mediate Subsequent Neutrophil Recruitment. J. Exp. Med. 200, 835-846. doi:10.1084/jem.20040501

Sulaiman, I., Greene, G., MacHale, E., Seheult, J., Mokoka, M., D’Arcy, S., et al. (2018). A Randomised Clinical Trial of Feedback on Inhaler Adherence and Technique in Patients with Severe Uncontrolled Asthma. Eur. Respir. J. 51, 51. doi:10.1183/13993003.01126-2017

Sverrild, A., Hansen, S., Hvidtfeldt, M., Clausson, C. M., Cozzolino, O., Cerps, S., et al. (2021). The Effect of Tezepelumab on Airway Hyperresponsiveness to Mannitol in Asthma (UPSTREAM). Eur. Respir. J. 28, 2101296. doi:10.1183/ 13993003.01296-2021

Upham, J. W., Le Lievre, C., Jackson, D. J., Masoli, M., Wechsler, M. E., and Price, D. B. (2021). Delphi Panel. Defining a Severe Asthma Super-Responder: Findings from a Delphi Process. J. Allergy Clin. Immunol. Pract. 9 (11), 3997-4004. doi:10.1183/13993003.congress-2020.2610

Varricchi, G., Bagnasco, D., Borriello, F., Heffler, E., and Canonica, G. W. (2016). Interleukin-5 Pathway Inhibition in the Treatment of Eosinophilic Respiratory Disorders: Evidence and Unmet Needs. Curr. Opin. Allergy Clin. Immunol. 16, 186-200. Available at: https://journals.lww.com/co-allergy/Fulltext/2016/04000/ Interleukin_5_pathway_inhibition_in_the_treatment.17.aspx. doi:10.1097/ ACI.0000000000000251

Wakashin, H., Hirose, K., Maezawa, Y., Kagami, S., Suto, A., Watanabe, N., et al. (2008). IL-23 and Th17 Cells Enhance Th2-Cell-Mediated Eosinophilic Airway Inflammation in Mice. Am. J. Respir. Crit. Care Med. 178, 1023-1032. doi:10.1164/rccm.200801-086OC

Walford, H. H., Lund, S. J., Baum, R. E., White, A. A., Bergeron, C. M., Husseman, J., et al. (2014). Increased ILC2s in the Eosinophilic Nasal Polyp Endotype Are Associated with Corticosteroid Responsiveness. Clin. Immunol. 155, 126-135. doi:10.1016/j.clim.2014.09.007

Wechsler, M., Ruddy, M. K., Pavord, I. D., Israel, E., Rabe, K. F., Abdulai, R. M., et al. (2020). SAR440340, an Anti-IL-33 Monoclonal Antibody, Demonstrated a Significant Reduction of LOAC Events and Improved Pre-BD FEV1 in Patients with Moderate to Severe Asthma: Results from the Phase 2 Proof of Concept Study. New Biological Treatments For Asthma, A4269-A4269. doi:10.1164/ ajrccm-conference.2020.201.1_meetingabstracts.a4269

Wechsler, M., Colice, G., Griffiths, J. M., Almqvist, G., Skärby, T., Piechowiak, T., et al. (2020). SOURCE: a Phase 3, Multicentre, Randomized, Double-Blind, Placebo-Controlled, Parallel Group Trial to Evaluate the Efficacy and Safety of Tezepelumab in Reducing Oral Corticosteroid Use in Adults with Oral Corticosteroid Dependent Asthma. Respir. Res. 21, 264. doi:10.1186/s12931020-01503-Z

Wechsler, M. E., Ruddy, M. K., Pavord, I. D., Israel, E., Rabe, K. F., Ford, L. B., et al. (2021). Efficacy and Safety of Itepekimab in Patients with Moderate-To-Severe Asthma. N. Engl. J. Med. 385, 1656-1668. doi:10.1056/nejmoa2024257

Weinstein, S. F., Katial, R. K., Bardin, P., Korn, S., McDonald, M., Garin, M., et al. (2019). Effects of Reslizumab on Asthma Outcomes in a Subgroup of Eosinophilic Asthma Patients with Self-Reported Chronic Rhinosinusitis with Nasal Polyps. J. Allergy Clin. Immunol. Pract. 7, 589. doi:10.1016/ j.jaip.2018.08.021

Wenzel, S., Castro, M., Corren, J., Maspero, J., Wang, L., Zhang, B., et al. (2016). Dupilumab Efficacy and Safety in Adults with Uncontrolled Persistent Asthma Despite Use of Medium-To-High-Dose Inhaled Corticosteroids Plus a LongActing $\beta 2$ Agonist: a Randomised Double-Blind Placebo-Controlled Pivotal Phase 2b Dose-Ranging Trial. Lancet 388, 31-44. doi:10.1016/S0140-6736(16) 30307-5

Wenzel, S., Ford, L., Pearlman, D., Spector, S., Sher, L., Skobieranda, F., et al. (2013). Dupilumab in Persistent Asthma with Elevated Eosinophil Levels. N. Engl. J. Med. 368, 2455-2466. doi:10.1056/ NEJMoa 1304048

Wenzel, S., Wilbraham, D., Fuller, R., Getz, E. B., and Longphre, M. (2007). Effect of an Interleukin-4 Variant on Late Phase Asthmatic Response to Allergen challenge in Asthmatic Patients: Results of 
Two Phase 2a Studies. Lancet 370, 1422-1431. doi:10.1016/S01406736(07)61600-6

Wright, A. K. A., Diver, S., Mccarthy, J., Marvin, A., Soares, M., Thornton, T., et al. (2019). Mepolizumab Does Not Alter the Blood Basophil Count in Severe Asthma. Allergy 74, 2488-2490. doi:10.1111/all.13879

Wright, T. K., Gibson, P. G., Simpson, J. L., McDonald, V. M., Wood, L. G., and Baines, K. J. (2016). Neutrophil Extracellular Traps Are Associated with Inflammation in Chronic Airway Disease. Respirology 21, 467-475. doi:10.1111/resp.12730

Wu, W., Samoszuk, M. K., Comhair, S. A., Thomassen, M. J., Farver, C. F., Dweik, R. A., et al. (2000). Eosinophils Generate Brominating Oxidants in AllergenInduced Asthma. J. Clin. Invest. 105 (10), 1455-1463. doi:10.1172/JCI9702

Yang, D., Guo, X., Liu, T., Li, Y., Du, Z., and Liu, C. (2021). Efficacy and Safety of Prostaglandin D2 Receptor 2 Antagonism with Fevipiprant for Patients with Asthma: a Systematic Review and Meta-Analysis of Randomized Controlled Trials. Curr. Allergy Asthma Rep. 21, 39. doi:10.1007/s11882-021-01017-8

Ying, S., Durham, S. R., Corrigan, C. J., Hamid, Q., and Kay, A. B. (1995). Phenotype of Cells Expressing mRNA for TH2-type (Interleukin 4 and Interleukin 5) and TH1-type (Interleukin 2 and Interferon Gamma) Cytokines in Bronchoalveolar Lavage and Bronchial Biopsies from Atopic Asthmatic and normal Control Subjects. Am. J. Respir. Cel Mol Biol 12, 477-487. doi:10.1165/ajrcmb.12.5.7742012

Ying, S., O'Connor, B., Ratoff, J., Meng, Q., Mallett, K., Cousins, D., et al. (2005). Thymic Stromal Lymphopoietin Expression Is Increased in Asthmatic Airways and Correlates with Expression of Th2-Attracting Chemokines and Disease Severity. J. Immunol. 174, 8183-8190. doi:10.4049/jimmunol.174.12.8183
Yu, Q. N., Guo, Y. B., Li, X., Li, C. L., Tan, W. P., Fan, X. L., et al. (2018). ILC2 Frequency and Activity Are Inhibited by Glucocorticoid Treatment via STAT Pathway in Patients with Asthma. Allergy 73, 1860-1870. doi:10.1111/all.13438

Conflict of Interest: MM reports grants from Canadian Institutes of Health Research, grants from Methapharm Specialty Pharmaceuticals, personal fees from AstraZeneca, personal fees from GlaxoSmithKline, outside the submitted work. PL reports funding from AstraZeneca and receives honoraria from AstraZeneca and GlaxoSmithKline.

The remaining author declares that that the research was conducted in the absence of any commercial or financial relationships that could be construed as a potential conflict of interest.

Publisher's Note: All claims expressed in this article are solely those of the authors and do not necessarily represent those of their affiliated organizations, or those of the publisher, the editors and the reviewers. Any product that may be evaluated in this article, or claim that may be made by its manufacturer, is not guaranteed or endorsed by the publisher.

Copyright (1) 2022 Salter, Lacy and Mukherjee. This is an open-access article distributed under the terms of the Creative Commons Attribution License (CC $B Y)$. The use, distribution or reproduction in other forums is permitted, provided the original author(s) and the copyright owner(s) are credited and that the original publication in this journal is cited, in accordance with accepted academic practice. No use, distribution or reproduction is permitted which does not comply with these terms. 